Supplementary Information for

\title{
Subcomponent Self-Assembly of Covalent Metallacycles Templated by Catalytically Active Seven-Coordinate Transition Metal Centers
}

Zhi-Wei Li, ${ }^{\dagger}$ Xin Wang, ${ }^{\dagger}$ Lian-Qiang Wei, ${ }^{\dagger}$ Ivana Ivanović-Burmazović,,$\stackrel{*}{\dagger}$ and Gao-Feng Liu* ${ }^{*} \dagger$

${ }^{\dagger}$ MOE Key Laboratory of Bioinorganic and Synthetic Chemistry, School of Chemistry, Sun Yat-Sen University, Guangzhou 510275, China.

${ }^{\ddagger}$ Department of Chemistry and Pharmacy, Friedrich-Alexander University Erlangen-Nuremberg, 91058 Erlangen, Germany.

E-mail: ivana.ivanovic-burmazovic@fau.de; liugaof@mail.sysu.edu.cn 


\section{Table of Contents}

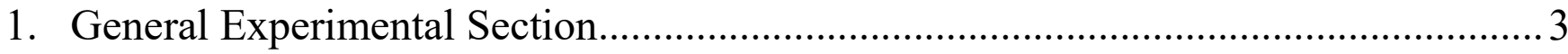

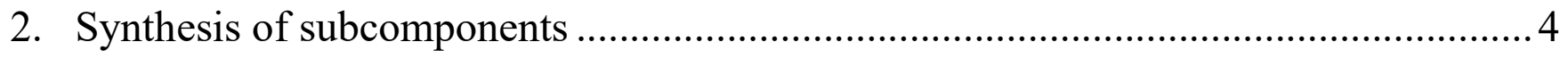

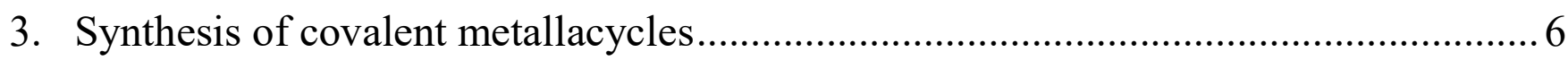

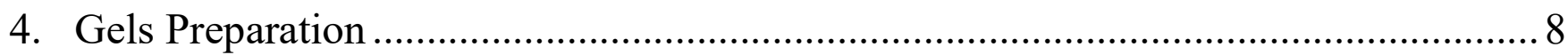

5. High resolution ESI-MS results of metallacycles 1-8 ............................................... 9

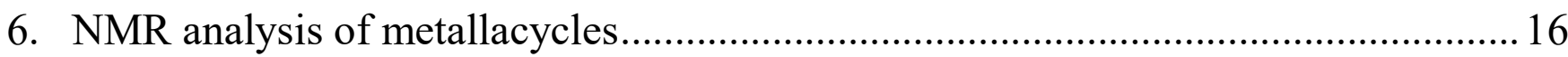

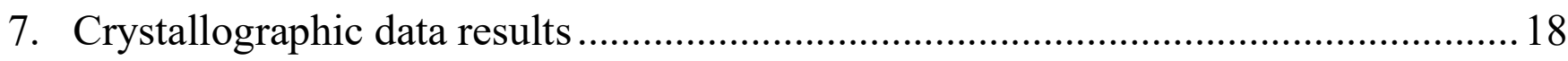

7.1 Response to the alerts in the crystallographic data................................................. 19

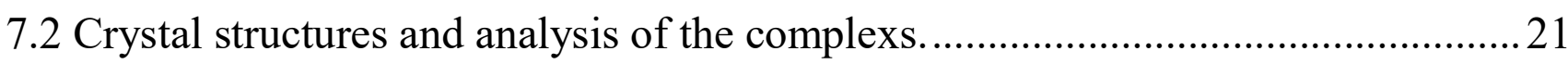

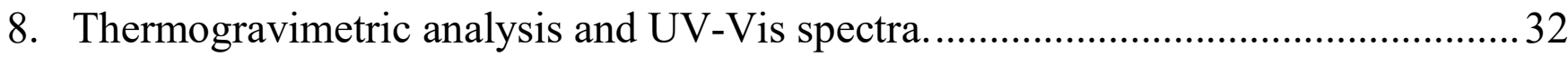

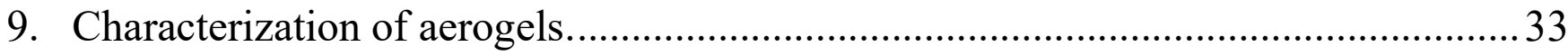

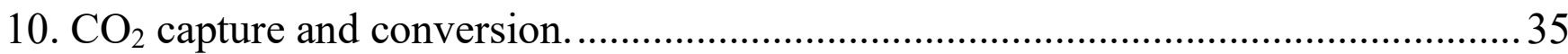

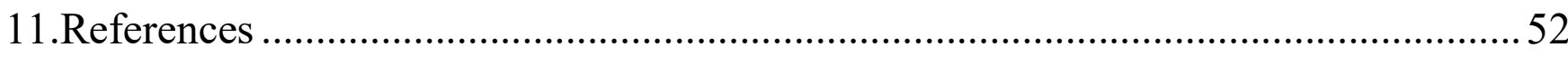




\section{General Experimental Section}

\section{Reagents and materials}

All starting materials and solvents, unless otherwise specified, were purchased from commercial sources and used as received. Methyl 2,6-diacetylisonicotinate was synthesized according to the reported procedures. ${ }^{\text {S1 }}$

\section{Measurements}

Nuclear magnetic resonance spectra $\left({ }^{1} \mathrm{H}\right.$ NMR and ${ }^{13} \mathrm{C}$ NMR) were obtained on a Bruker Ascend $300 \mathrm{or} 400 \mathrm{MHz}$ spectrometers at ambient temperature. ${ }^{1} \mathrm{H}$ NMR spectra are referred to the TMS signal and ${ }^{13} \mathrm{C}$ NMR spectra are referred to the residual solvent signal. Data for ${ }^{1} \mathrm{H}$ NMR are reported as follows: chemical shifts $(\delta \mathrm{ppm})$, multiplicities $(\mathrm{s}=\mathrm{singlet}, \mathrm{d}=$ doublet, $\mathrm{t}=$ triplet, $\mathrm{q}=$ quartet, $\mathrm{m}=$ multiplet, $\mathrm{br}=$ broad $)$, coupling constants $(\mathrm{Hz})$, integration.

Low resolution mass spectra of subcomponents were recorded on Thermo Fisher Scientific LTQ XL. High resolution electrospray ionization mass spectrometry (HRMS) experiments were performed on a Thermo Scientific LTQ Orbitrap Fusion Lumos instrument. Samples were diluted in methanol.

IR spectra were recorded in the range of $4000-450 \mathrm{~cm}^{-1}$ on recorded on Bruker FT IR spectrometer (EQUINOX 55) using the $\mathrm{KBr}$ disc technique. UV/Vis spectra were recorded on a Perkin Elmer Lambda 950.

Thermogravimetric analysis (TGA) were performed on a NETZSCH TG209 system under $\mathrm{N}_{2}$ atmosphere in the range of $30-800{ }^{\circ} \mathrm{C}$ at a heating rate of $10{ }^{\circ} \mathrm{C} \mathrm{min}^{-1}$.

Powder X-ray diffraction (PXRD) data were recorded on a Bruker D8 powder X-ray diffractometer with Cu-K $\alpha$ radiation $(\lambda=1.5406 \AA)$.

Inductively coupled plasma optical emission spectrometry (ICP-OES) analysis was measured by PerkinElmer Optima 8300 ICP-OES spectrometers.

$\mathrm{N}_{2}$ adsorption measurements were carried out using a ASAP2460 analyzer. Prior to an adsorption measurement, aerogel was typically degassed at $100{ }^{\circ} \mathrm{C}$ for $12 \mathrm{~h}$ to remove solvent molecules within the network. The sorption isotherms for $\mathrm{CO}_{2}$ was measured with an automatic volumetric sorption apparatus Micromertics ASAP 2020M. Before each sorption experiment, the sample was heated at $100{ }^{\circ} \mathrm{C}$ under high vacuum for $6 \mathrm{~h}$ to remove the remnant solvent molecules.

The morphologies and nanostructure were recorded by FE-SEM (SU8010) and TEM (Tecnai G2 F30). Elemental distribution was examined by Thermal Field Emission Environmental energy dispersive spectroscopy (Quanta 400F).

Single-Crystal X-ray Crystallography. The X-ray diffraction data were collected on an Agilent Sapphire3 Gemini Ultra single crystal diffractometer using $\mathrm{Cu}$ radiation $(\lambda=1.54184$ ) at $150.15 \mathrm{~K}$ (for 1), an Agilent Gemini S Ultra CCD Diffractometer with the Enhance X-ray Source of $\mathrm{Cu}$ radiation $(\lambda=1.54178 \AA)$ using the $\omega-\phi$ scan technique at $150.00(10) \mathrm{K}$ (for 3) and a Bruker D8 QUEST diffractometer with $\operatorname{MoK} \alpha(\lambda=0.71073 \AA$ ) radiation at $120 \mathrm{~K}$ (for 2, 4, 6). The crystals employed immediately lost solvent after removal from the mother liquor and rapid handling prior to flash cooling in the cryostream was required to collect data. The intensity data were corrected for Lorentz and polarization effects (SAINT), and empirical absorption corrections based on equivalent reflections were applied (SADABS). ${ }^{\mathrm{S} 2}$ The structures were solved by direct methods and refined by the full-matrix least-squares method on $\mathrm{F}^{2}$ with SHELXTL program package. ${ }^{\mathrm{S} 3}$ All nonhydrogen atoms were refined with anisotropic displacement parameters. The hydrogen atoms were calculated and refined as a riding model. The hydrogen atoms of the water molecules were located from difference maps. All the electrons of disordered solvent molecules which cannot be determined, are removed by SQUEEZE routine of PLATON program. 


\section{Synthesis of subcomponents}

Scheme S1. synthetic scheme for Methyl 2,6-diacetylisonicotinate and Methyl 2,6-diacetylisonicotinate.<smiles>COC(=O)c1ccncc1</smiles><smiles>CC#CCC#CC(C)=O</smiles><smiles>C#CC</smiles>

S1<smiles>CC(=O)c1cc(C(C)=O)nc(C(=O)O)c1</smiles>

S2

Reagents and Conditions: (i) pyruvic acid, $\mathrm{AgNO}_{3}$, R.T $\left(\mathrm{NH}_{4}\right)_{2} \mathrm{~S}_{2} \mathrm{O}_{8}$ (ii) LiOH, $1 \mathrm{M} \mathrm{HCl}, 60{ }^{\circ} \mathrm{C}$.<smiles>COC(=O)c1cc(C(C)=O)nc(C(C)=O)c1</smiles>

S1

Methyl 2,6-diacetylisonicotinate(S1) was synthesized using a modified literature procedure. To a solution of $\mathrm{H}_{2} \mathrm{SO}_{4}(0.4 \mathrm{M}$, $500 \mathrm{~mL}$ ) was added methyl isonicotinate $(5.50 \mathrm{~g}, 40 \mathrm{mmol})$, pyruvic acid $(14.1 \mathrm{~g}, 140 \mathrm{mmol})$, and $\mathrm{AgNO}_{3}(0.54 \mathrm{~g}$ in $5 \mathrm{~mL}$ of water). Then ammonium persulfate $(36.5 \mathrm{~g}, 160 \mathrm{mmol})$ was added in small portions to the mixture. The mixture was stirred at room temperature overnight and extracted with dichloromethane $(3 \times 100 \mathrm{~mL})$. The organic phase was washed with a saturated $\mathrm{Na}_{2} \mathrm{CO}_{3}$ solution $(100 \mathrm{~mL})$ and brine $(100 \mathrm{~mL})$, then dried over $\mathrm{MgSO}_{4}$. Evaporation of the solvent gave rude product. Recrystallization from methanol gave white crystalline needles of methyl 2,6-diacetylisonicotinate $(2.5 \mathrm{~g}, 28.6 \%) .{ }^{1} \mathrm{H}$ NMR $\left(400 \mathrm{MHz}, \mathrm{DMSO}-\mathrm{d}_{6}\right) \delta 8.47(\mathrm{~s}, 2 \mathrm{H}), 3.96(\mathrm{~s}, 3 \mathrm{H}), 2.76(\mathrm{~s}, 6 \mathrm{H}) .{ }^{13} \mathrm{C}$ NMR $\left(101 \mathrm{MHz}, \mathrm{DMSO}-\mathrm{d}_{6}\right) \delta 197.94,163.98,153.38$, 139.64, 122.76, 53.22, 25.43. ESI-MS (m/z): calcd for $\mathrm{C}_{11} \mathrm{H}_{11} \mathrm{NO}_{4}, 221.07$. Found: $\left[\mathrm{C}_{11} \mathrm{H}_{11} \mathrm{NO}_{4}+\mathrm{Na}^{+}\right]^{+}, 244.30$.<smiles>CC(=O)c1cc(C(C)=O)nc(C(=O)O)c1</smiles>

S2

Methyl 2,6-diacetylisonicotinate $(5.0 \mathrm{mmol}, 1.1 \mathrm{~g})$ and $\mathrm{NaOH}(5.1 \mathrm{mmol}, 0.21 \mathrm{~g})$ were dissolved in $10 \mathrm{~mL}$ mixed solvent (methanol/water $=4: 1$ ). The mixture was stirred at $60^{\circ} \mathrm{C}$, monitored by TLC (petroleum ether / ethyl acetate $=8: 1$ ). Acidify the mixture to $\mathrm{pH}=4$ by $1 \mathrm{M} \mathrm{HCl}$ and extracted with Ethyl acetate $(3 \times 10 \mathrm{~mL})$. The organic layer was washed with saturated aqueous sodium chloride, then dried over magnesium sulfate and concentrated to give 2,6-diacetylisonicotinic acid (S2) as a solid (0.98 g, 95\%). ${ }^{1} \mathrm{H}$ NMR (400 MHz, DMSO-d 6$) \delta 14.19$ (s, 1H), 8.47 (s, 2H), 2.76 (s, 6H). ${ }^{13} \mathrm{C}$ NMR (101 MHz, DMSO$\left.\mathrm{d}_{6}\right) \delta 198.10,164.96,153.37,141.04,123.04,25.49$. ESI-MS (m/z): calcd for $\mathrm{C}_{10} \mathrm{H}_{8} \mathrm{NO}_{4}^{-}, 206.18$ and $\left[2 \mathrm{C}_{10} \mathrm{H}_{9} \mathrm{NO}_{4}-\mathrm{H}\right]^{-}, 413.36$. Found: 206.05 and 413.10. 


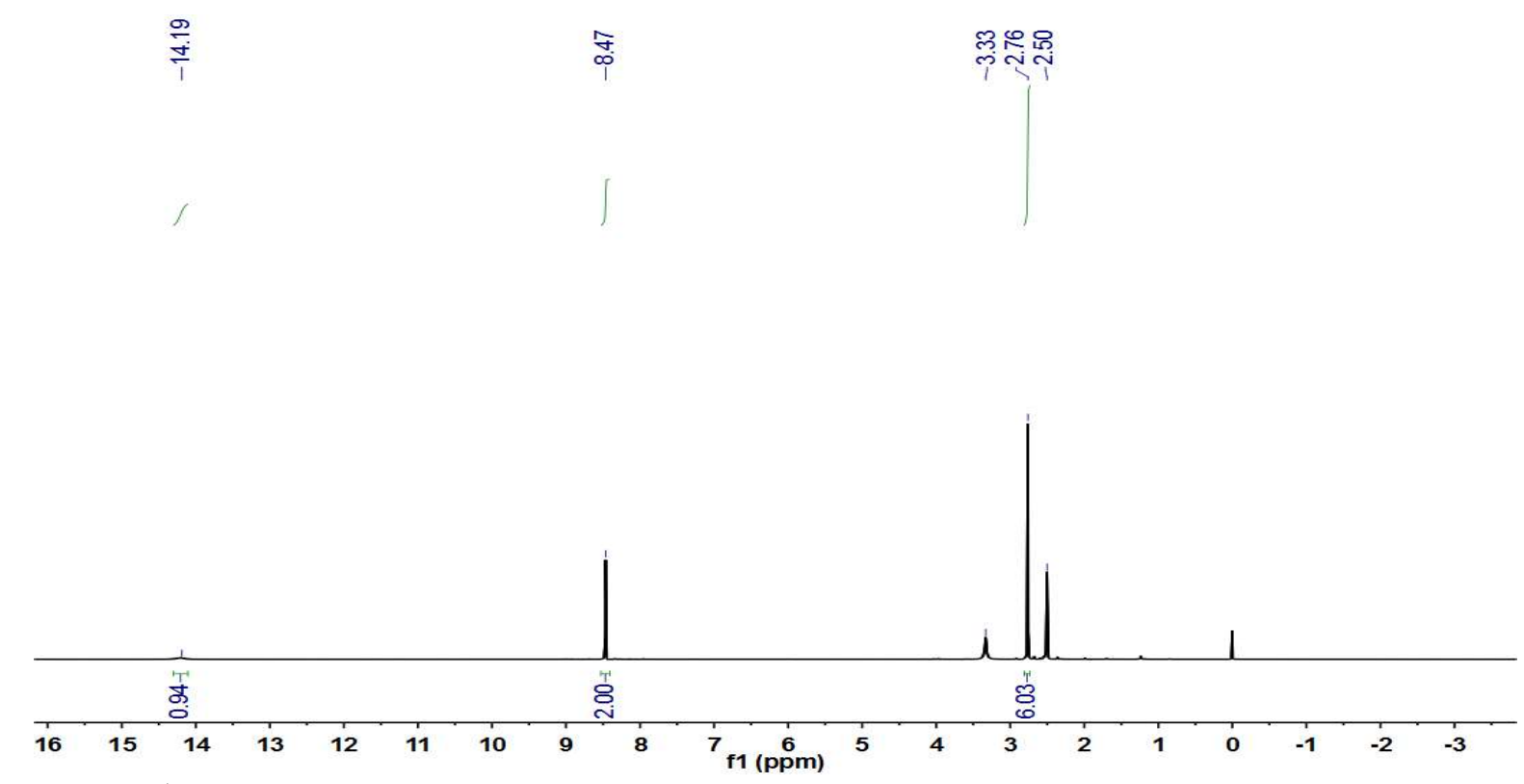

Figure S1. ${ }^{1} \mathrm{H}$ NMR spectrum of $\mathbf{S 2}\left(400 \mathrm{MHz}, \mathrm{DMSO}_{-} \mathrm{d}_{6}, 298 \mathrm{~K}\right)$.

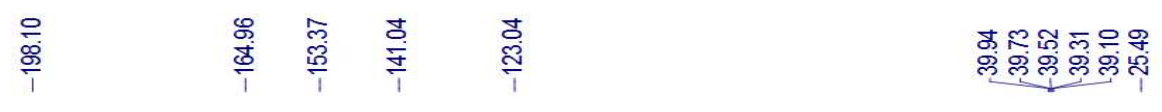

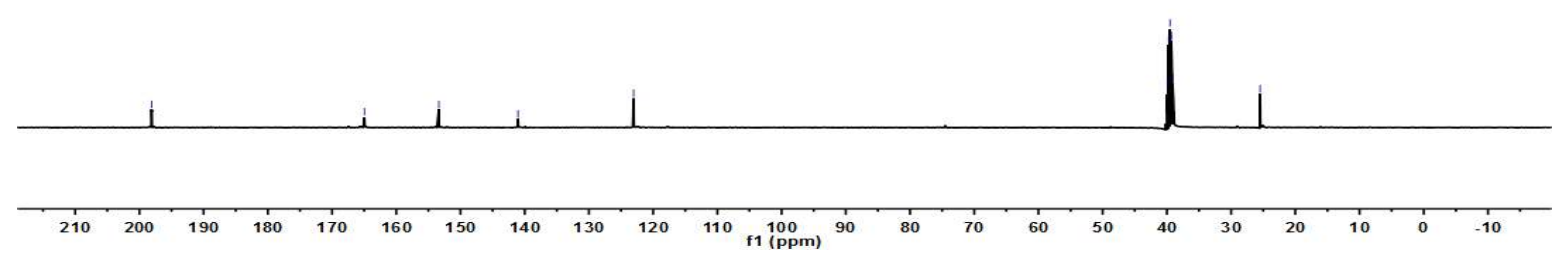

Figure S2. ${ }^{13} \mathrm{C}$ NMR spectrum of $\mathbf{S 2}\left(101 \mathrm{MHz}, \mathrm{DMSO}_{-} \mathrm{d}_{6}, 298 \mathrm{~K}\right)$.

Scheme S2. synthetic scheme for 1,1'-Biphenyl-4,4'-dicarbohydrazide.

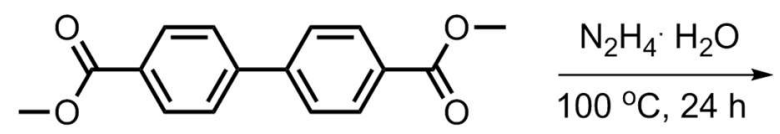

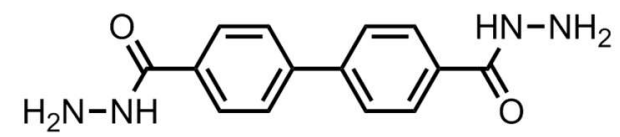

S3

1,1'-Biphenyl-4,4'-dicarbohydrazide(S3) was synthesized using a modified literature procedure. ${ }^{\mathrm{S} 4} \mathrm{~A}$ round bottom flask equipped with stir bar was added dimethyl 4,4'-biphenyl dicarboxylate $(0.54 \mathrm{~g}, 2.0 \mathrm{mmol})$ and excessive hydrazine monohydrate $(10.0 \mathrm{~mL})$. This was heated at $100^{\circ} \mathrm{C}$ for $24 \mathrm{~h}$, followed by cooling. The product was filtered and washed with water and methanol without further purification, then the obtained solid was dried to give $\mathbf{S 3}$ as a white powder $(0.50 \mathrm{~g}, 92.6 \%)$. ${ }^{1} \mathrm{H}$ NMR $\left(400 \mathrm{MHz}\right.$, DMSO-d 6 ) $\delta 9.85(\mathrm{~s}, 1 \mathrm{H}), 7.93(\mathrm{~d}, \mathrm{~J}=7.8 \mathrm{~Hz}, 2 \mathrm{H}), 7.81(\mathrm{~d}, \mathrm{~J}=7.8 \mathrm{~Hz}, 2 \mathrm{H}), 4.52(\mathrm{~s}, 2 \mathrm{H}) .{ }^{13} \mathrm{C}$ NMR $(101$ MHz, DMSO-d 6 ) $\delta 165.89,142.04,133.04,128.12,127.19$. 


\section{Synthesis of covalent metallacycles}

Scheme S3. synthetic scheme for covalent metallacycles.
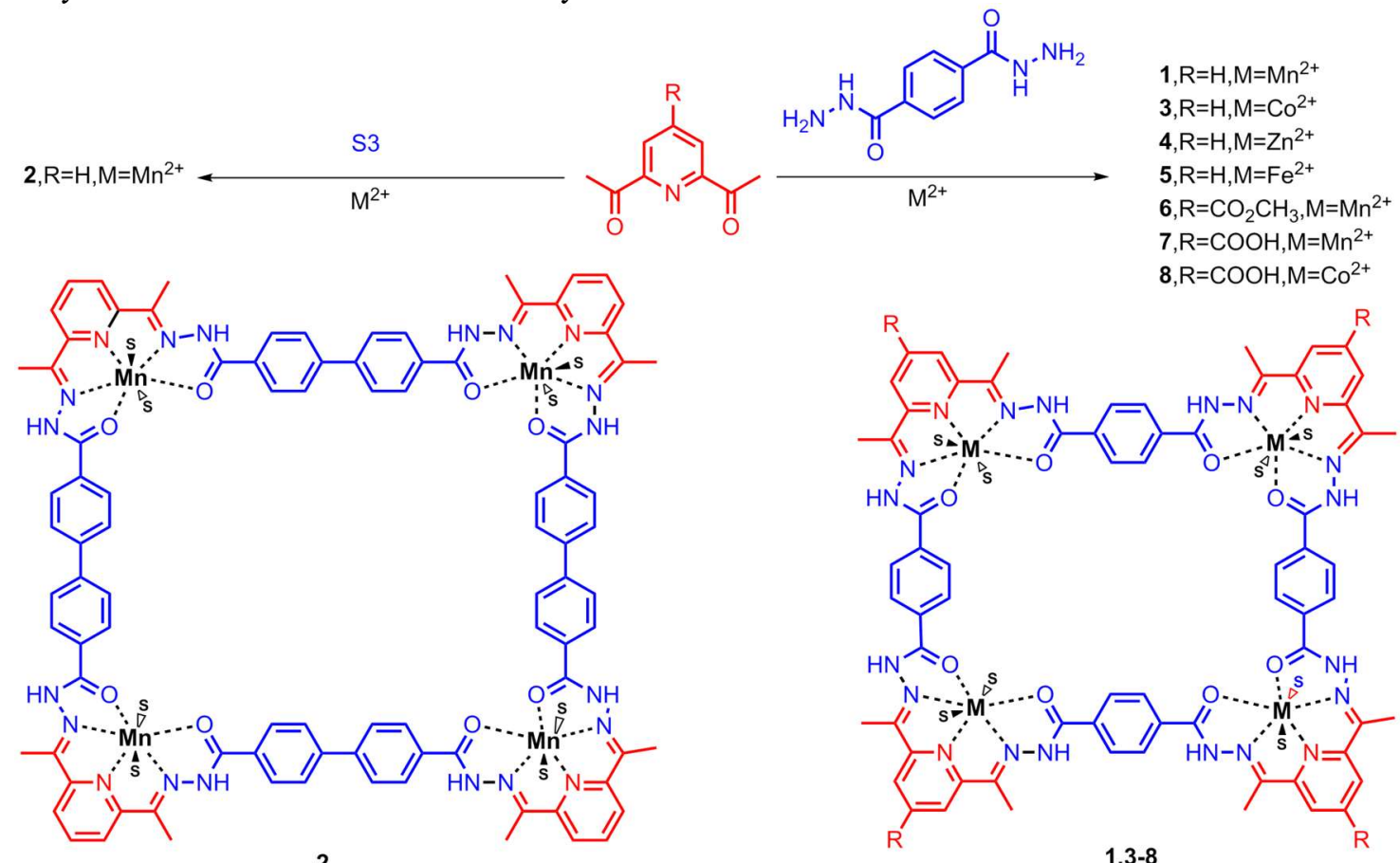

1: 2,6-Diacetylpyridine (2.0 mmol, $326.0 \mathrm{mg})$, Terephthalic dihydrazide $(2.0 \mathrm{mmol}, 388.0 \mathrm{mg})$, and $\mathrm{Mn}\left(\mathrm{ClO}_{4}\right)_{2} \cdot 6 \mathrm{H}_{2} \mathrm{O}(2.0$ mmol, $724.0 \mathrm{mg})$ were suspended in DMF $(10 \mathrm{~mL})$ and heated at $100{ }^{\circ} \mathrm{C}$ for 24 hours. After cooling to room temperature, diethyl ether $(20 \mathrm{~mL})$ was added to the solution and the resulting precipitate was collected by centrifugation. Drying under

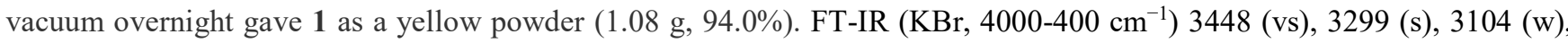
2939 (w), 1629 (vs), 1567 (m), 1529 (s), 1494 (m), 1457 (m), 1440 (m), 1376 (vs), 1292 (vs), 1174 (vs), 1095 (vs), 1014 (m), $896(\mathrm{w}), 815(\mathrm{w}), 725(\mathrm{w}), 671(\mathrm{w}), 624(\mathrm{~s}), 559(\mathrm{w}), 536(\mathrm{w})$.

1': X-ray-quality single crystals were grown by layer-by-layer slow diffusion. Concretely, a solution of 1 in $\mathrm{CH}_{3} \mathrm{OH}(2 \mathrm{~mL})$ was placed into the bottom of the tube, and $2 \mathrm{ml} \mathrm{H}_{2} \mathrm{O}$ was carefully layered on top of $\mathbf{1}$, finally $2 \mathrm{ml}$ aqueous $\mathrm{NaOH}$ solution $(0.1 \mathrm{M})$ was carefully layered on top of water. The solution was allowed to stand for one week, resulting in the formation of red block-shaped crystals.

2: 2,6-Diacetylpyridine (0.5 mmol, $81.5 \mathrm{mg}), 1,1^{\prime}$-Biphenyl-4,4'-dicarbohydrazide (0.5 mmol, $\left.135.0 \mathrm{mg}\right)$, and $\mathrm{Mn}\left(\mathrm{ClO}_{4}\right)_{2} \cdot 6 \mathrm{H}_{2} \mathrm{O}(0.5 \mathrm{mmol}, 181.0 \mathrm{mg})$ were suspended in DMF $(6 \mathrm{~mL})$ and heated at $100{ }^{\circ} \mathrm{C}$ for 24 hours. After cooling to room temperature, diethyl ether $(20 \mathrm{~mL})$ was added to the solution and the resulting precipitate was collected by centrifugation. Drying under vacuum overnight gave 2 as a yellow powder (310.0 mg, 95.2\%). FT-IR (KBr, 4000-400 cm $\mathrm{cm}^{-1} 3243$ (vs), 3104 (s), $2933(\mathrm{w}), 2618(\mathrm{w}), 2256(\mathrm{w}), 1623$ (vs), $1610(\mathrm{vs}), 1571(\mathrm{~m}), 1527(\mathrm{~s}), 1488(\mathrm{~s}), 1456(\mathrm{~m}), 1438(\mathrm{~m}), 1378(\mathrm{~m}), 1284(\mathrm{~s})$, $1170(\mathrm{~m}), 1087(\mathrm{vs}), 1006(\mathrm{~m}), 896(\mathrm{w}), 750(\mathrm{w}), 669(\mathrm{w}), 624(\mathrm{~s}), 561(\mathrm{w}), 507(\mathrm{w})$.

2':X-ray-quality single crystals were grown by slow diffusion of pyridine into a DMF solution of 2 (5 mg sample was dissolved in $10 \mathrm{~mL}$ DMF).

3: 2,6-Diacetylpyridine $(2.0 \mathrm{mmol}, 326.0 \mathrm{mg})$, Terephthalic dihydrazide $(2.0 \mathrm{mmol}, 388.0 \mathrm{mg})$, and $\mathrm{Co}\left(\mathrm{ClO}_{4}\right)_{2} \cdot 6 \mathrm{H}_{2} \mathrm{O}(2.0$ mmol, $732.0 \mathrm{mg}$ ) were suspended in methanol $(100 \mathrm{~mL})$ and heated at $85{ }^{\circ} \mathrm{C}$ for 24 hours. After concentrating into $5 \mathrm{~mL}$ by rotary evaporation, diethyl ether $(20 \mathrm{~mL})$ was added to the solution and the resulting precipitate was collected by centrifugation. Drying under vacuum overnight gave 3 as an orange-yellow powder (1.1 g, 95.0\%). X-ray-quality single crystals were grown by slow diffusion of benzene into a methanol / acetonitrile (1:1) solution. FT-IR (KBr, 4000-400 cm $\left.{ }^{-1}\right) 3425$ (vs), 3245 (vs), 3106 (vs), 3018 (vs), 2939 (vs), 2840 (vs), 2021 (m), 1629 (vs), 1567 (s), 1529 (vs), 1494 (s), 1442 (m), 1378 (m), 1295 (s), 1182 (m), 1087 (vs), 1018 (m), 925 (w), 898 (w), 863 (w), 809 (w), $725(\mathrm{w}), 673$ (w), $622(\mathrm{~s}), 572(\mathrm{w}), 536(\mathrm{w})$.

4: 2,6-Diacetylpyridine (1.0 mmol, $163.0 \mathrm{mg})$, Terephthalic dihydrazide (1.0 mmol, $194.0 \mathrm{mg})$, and $\mathrm{Zn}\left(\mathrm{CF}_{3} \mathrm{SO}_{3}\right)_{2}(1.0 \mathrm{mmol}$, $363.5 \mathrm{mg}$ ) were suspended in methanol $(50 \mathrm{~mL})$ and heated at $85^{\circ} \mathrm{C}$ for 12 hours. After concentrating into $5 \mathrm{~mL}$ by rotary evaporation, diethyl ether $(20 \mathrm{~mL})$ was added to the solution and the resulting precipitate was collected by centrifugation. Drying under vacuum overnight gave 4 as a yellow powder $(618.0 \mathrm{mg}, 90.0 \%)$. X-ray-quality single crystals were grown by slow diffusion of diethyl ether into an acetonitrile solution. ${ }^{1} \mathrm{H}$ NMR $\left(300 \mathrm{MHz}, \mathrm{CD}_{3} \mathrm{CN}\right) \delta 11.39(\mathrm{br}, 2 \mathrm{H}), 8.45(\mathrm{~s}, 4 \mathrm{H}), 8.40$ 
$(\mathrm{t}, \mathrm{J}=7.9 \mathrm{~Hz}, 1 \mathrm{H}), 8.21(\mathrm{~d}, \mathrm{~J}=7.8 \mathrm{~Hz}, 2 \mathrm{H}), 2.77(\mathrm{~s}, 6 \mathrm{H}) .{ }^{13} \mathrm{C}$ NMR (101 MHz, DMSO) $\delta 167.70,149.70,147.72,143.13$, 134.52, 129.38, 125.61 (q, J=323.2 Hz), 125.14, 14.16. FT-IR (KBr, 4000-400 cm c $^{-1} 3446$ (vs), 3249 (s), 3020 (m), 1637 (vs), 1535 (vs), 1496 (m), 1459 (m), 1444 (m), 1380 (m), 1284 (vs), 1255 (vs), 1176 (s), 1079 (s), 1029 (vs), 898 (w), 865 (w), 813 (w), $782(\mathrm{w}), 761(\mathrm{w}), 640(\mathrm{~s}), 574(\mathrm{w}), 516(\mathrm{w})$.

5: 2,6-Diacetylpyridine $(0.5 \mathrm{mmol}, 81.5 \mathrm{mg})$, Terephthalic dihydrazide $(0.5 \mathrm{mmol}, 97.0 \mathrm{mg})$, and $\mathrm{Fe}\left(\mathrm{CF}_{3} \mathrm{SO}_{3}\right)_{2}(0.5 \mathrm{mmol}$, $177.0 \mathrm{mg}$ ) were suspended in methanol $(50 \mathrm{~mL})$ and stirred at room temperature for 24 hours. After concentrating into $5 \mathrm{~mL}$ by rotary evaporation, diethyl ether $(20 \mathrm{~mL})$ was added to the solution and the resulting precipitate was collected by centrifugation. Drying under vacuum overnight gave 5 as a green powder $(224.0 \mathrm{mg}, 66.0 \%)$. X-ray-quality single crystals were grown by slow diffusion of diethyl ether into an acetonitrile solution. FT-IR (KBr, 4000-400 cm ${ }^{-1}$ ) 3425 (vs), 3212 (vs), 3020 (vs), 2846 (s), 2400 (m), 1629 (vs), 1567 (vs), 1537 (vs), 1496 (s), 1438 (s), 1382 (s), 1278 (vs), 1253 (vs), 1168 (s), $1079(\mathrm{~m}), 1029(\mathrm{vs}), 900(\mathrm{w}), 865(\mathrm{w}), 811(\mathrm{w}), 725(\mathrm{w}), 640(\mathrm{~s}), 574(\mathrm{~m}), 545(\mathrm{~m}), 514(\mathrm{~m})$.

6: Methyl 2,6-diacetylisonicotinate $(0.5 \mathrm{mmol}, 110.5 \mathrm{mg})$, Terephthalic dihydrazide $(0.5 \mathrm{mmol}, 97.0 \mathrm{mg})$, and $\mathrm{Mn}\left(\mathrm{ClO}_{4}\right)_{2} \cdot 6 \mathrm{H}_{2} \mathrm{O}(0.5 \mathrm{mmol}, 181.0 \mathrm{mg})$ were suspended in DMF $(10 \mathrm{~mL})$ and heated at $100{ }^{\circ} \mathrm{C}$ for 12 hours. After cooling to room temperature, diethyl ether $(20 \mathrm{~mL})$ was added to the solution and the resulting precipitate was collected by centrifugation. Drying under vacuum overnight gave 6 as a red powder (238.5 mg, 75.4\%). FT-IR (KBr, 4000-400 cm $\left.{ }^{-1}\right) 3405$ (vs), 3014 (m), 2958 (m), 1727 (s), 1629 (vs), 1565 (m), 1531 (s), 1494 (m), 1436 (m), 1371 (m), 1294 (vs), 1265 (s), 1172 (s), 1139 (m), 1085 (vs), 1014 (m), 975 (m), 904 (w), 765 (w), $721(\mathrm{w}), 676(\mathrm{w}), 626(\mathrm{~s}), 553(\mathrm{w})$.

6': X-ray-quality single crystals were grown by slow diffusion of pyridine into an acetonitrile solution of 6 (5 mg sample was dissolved in $10 \mathrm{~mL}$ acetonitrile).

7: 2,6-diacetylisonicotinic acid $(0.5 \mathrm{mmol}, 103.5 \mathrm{mg})$, Terephthalic dihydrazide $(0.5 \mathrm{mmol}, 97.0 \mathrm{mg})$, and $\mathrm{Mn}\left(\mathrm{ClO}_{4}\right)_{2} \cdot 6 \mathrm{H}_{2} \mathrm{O}$ $(0.5 \mathrm{mmol}, 181.0 \mathrm{mg})$ were suspended in DMF $(10 \mathrm{~mL})$ and heated at $100{ }^{\circ} \mathrm{C}$ for 12 hours. After cooling to room temperature, diethyl ether $(20 \mathrm{~mL})$ was added to the solution and the resulting precipitate was collected by centrifugation. Drying under vacuum overnight gave 7 as a yellow powder (280.0 mg, 90.3\%). FT-IR (KBr, 4000-400 cm ch $^{-1} 3399$ (vs), 3239 (vs), 3002 (s), 2971 (s), 2933 (s), 2819 (m), 1652 (vs), 1633 (vs), 1537 (s), 1494 (m), 1434 (m), 1417 (m), 1386 (s), 1294 (s), 1178 (m), 1145 (s), 1099 (s), $1018(\mathrm{~m}), 916(\mathrm{w}), 727(\mathrm{w}), 669(\mathrm{w}), 624(\mathrm{~m})$.

8: 2,6 -diacetylisonicotinic acid $(0.5 \mathrm{mmol}, 103.5 \mathrm{mg})$, Terephthalic dihydrazide $(0.5 \mathrm{mmol}, 97.0 \mathrm{mg})$, and $\mathrm{Co}\left(\mathrm{ClO}_{4}\right)_{2} \cdot 6 \mathrm{H}_{2} \mathrm{O}$ $(0.5 \mathrm{mmol}, 188.0 \mathrm{mg})$ were suspended in DMF $(10 \mathrm{~mL})$ and heated at $100{ }^{\circ} \mathrm{C}$ for 12 hours. After cooling to room temperature, diethyl ether $(20 \mathrm{~mL})$ was added to the solution and the resulting precipitate was collected by centrifugation. Drying under vacuum overnight gave 8 as a red powder $\left(250.0 \mathrm{mg}, 80.0 \%\right.$ ). FT-IR (KBr, 4000-400 $\left.\mathrm{cm}^{-1}\right) 3363$ (vs), 3214 (vs), 3006 (s), 2933 (s), 1629 (vs), 1537 (vs), 1494 (m), 1430 (m), 1388 (s), 1295 (s), 1184 (m), 1143 (m), 1093 (vs), 1018 (m), 917 (w), 867 (w), $750(\mathrm{w}), 676(\mathrm{w}), 622(\mathrm{~m}), 572(\mathrm{w}), 542(\mathrm{w})$. 


\section{Gels Preparation}

Scheme S4. synthetic scheme for aerogels.

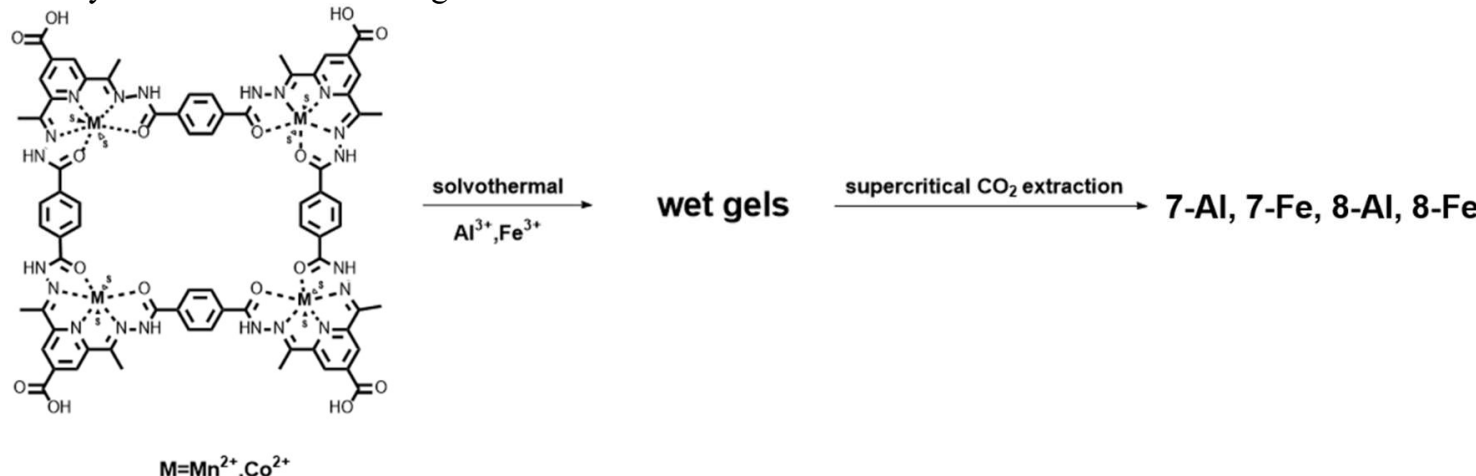

4.1 Typical procedure for 7-Al and 7-Fe: $7(100.0 \mathrm{mg}, 0.04 \mathrm{mmol})$ and $\mathrm{Al}\left(\mathrm{NO}_{3}\right)_{3} \cdot 9 \mathrm{H}_{2} \mathrm{O}(60.0 \mathrm{mg}, 0.16$ mmol $)$ were dissolved in $2 \mathrm{ml}$ of DMF and $2 \mathrm{ml}$ of EtOH, respectively. A yellow solution was obtained after mixing them in a vial, and kept at $90{ }^{\circ} \mathrm{C}$ in an oven for 24 hours. The yellow wet gel was obtained. In the same way, when $\mathrm{Al}\left(\mathrm{NO}_{3}\right)_{3} \cdot 9 \mathrm{H}_{2} \mathrm{O}$ was replaced by $\mathrm{Fe}\left(\mathrm{NO}_{3}\right)_{3} \cdot 9 \mathrm{H}_{2} \mathrm{O}$, the dark green wet gel was obtained. After gelation, the wet gels were subjected to solvent exchange with EtOH $(5 \mathrm{ml} \times 5)$. The gels were then placed in a high-pressure Soxhlet stainless-steel autoclave extractor $(0.75 \mathrm{~L})$. The solvent in the wet gels was extracted with liquid $\mathrm{CO}_{2}$ (ca. $270 \mathrm{~g}$ ) for $24 \mathrm{~h}$, and the extraction temperature was kept at $35.0{ }^{\circ} \mathrm{C}$ (pressure 5.9 MPa). After the extractors were depressurized slowly at room temperature over ca. 2-3 h, two aerogels were obtained, denoted as 7-Al $(75.0 \mathrm{mg})$, 7-Fe $(81.5 \mathrm{mg})$, respectively. FT-IR of 7-Al $\left(\mathrm{KBr}, 4000-400 \mathrm{~cm}^{-1}\right)$ 3402 (vs), 2964 (vs), 2931 (s), 1628 (s), 1543 (vs), 1495 (vs), 1385 (m), 1329 (m), 1294 (s), 1180 (s), 1147 (m), 1115 (m), 1090 (vs), 1016 (m), 917 (w), 791 (w), 750 (w), 727 (w), 676 (m), 630 (w), 565 (w). FT-IR of 7-Fe $\left(\mathrm{KBr}, 4000-400\right.$ cm $\left.{ }^{-1}\right)$ 3392 (vs), 2971 (vs), 2929 (s), 1620 (s), 1551 (vs), 1506 (vs), 1473 (m), 1365 (m), 1298 (s), 1174 (s), 1110 (m), 1068 (m), $1014(\mathrm{vs}), 788(\mathrm{w}), 730(\mathrm{w}), 696(\mathrm{~m}), 557(\mathrm{w})$.

4.2 8-Al and 8-Fe: $8(100.0 \mathrm{mg}, 0.04 \mathrm{mmol})$ and $\mathrm{Al}\left(\mathrm{NO}_{3}\right)_{3} \cdot 9 \mathrm{H}_{2} \mathrm{O}(60.0 \mathrm{mg}, 0.16 \mathrm{mmol})$ were dissolved in $2 \mathrm{ml}$ of $\mathrm{DMF}$ and $2 \mathrm{ml}$ of EtOH, respectively. A red solution was obtained after mixing them in a vial, and kept at $90{ }^{\circ} \mathrm{C}$ in an oven for 24 hours. The red wet gel was obtained. In the same way, when $\mathrm{Al}\left(\mathrm{NO}_{3}\right)_{3} \cdot 9 \mathrm{H}_{2} \mathrm{O}$ was replaced by $\mathrm{Fe}\left(\mathrm{NO}_{3}\right)_{3} \cdot 9 \mathrm{H}_{2} \mathrm{O}$, the dark green wet gel was obtained. 8-Al $(80.0 \mathrm{mg})$ and 8-Fe $(85.0 \mathrm{mg})$ aerogels were prepared following similar procedure. FTIR of 8-Al (KBr, 4000-400 cm cl) $^{-1} 396$ (vs), 2964 (vs), 2931 (s), 1621 (s), 1549 (vs), 1495 (vs), 1385 (m), 1317 (m), 1288 (s), 1184 (s), 1147 (m), 1115 (m), 1016 (vs), 921 (w), 867 (w), 829 (w), 790 (w), 728 (m), 688 (w), 629 (w). FT-IR of 8Fe (KBr, 4000-400 cm cm $^{-1} 3378$ (vs), 2987 (vs), 2925 (s), 1614 (s), 1550 (vs), 1508 (vs), 1475 (m), 1367 (m), 1294 (s), 1176 (s), 1153 (m), 1110 (m), 1068 (vs), 1016 (w), 788 (w), 730 (w), 696 (w), 628 (m), 592 (w), 555 (w). 
5. High resolution ESI-MS results of metallacycles 1-8.

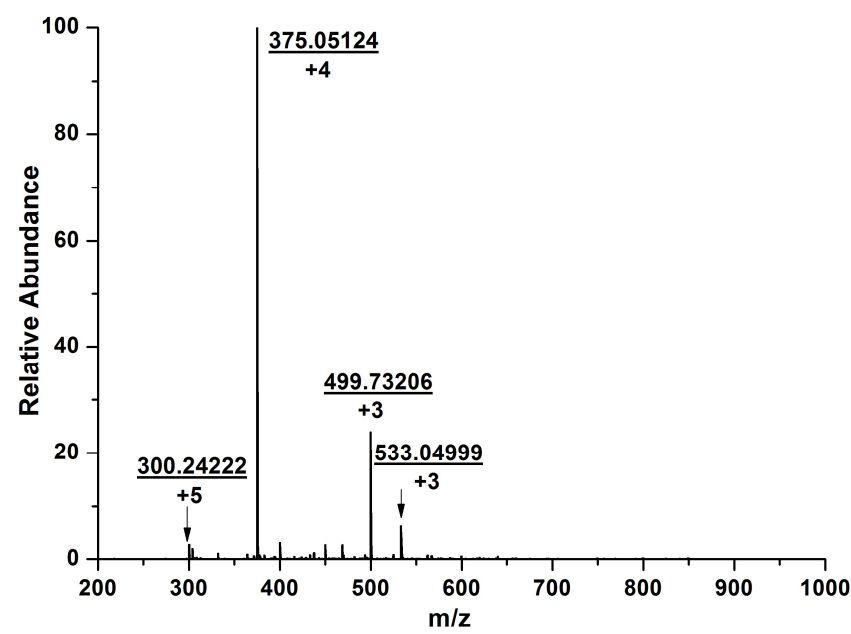

(a)

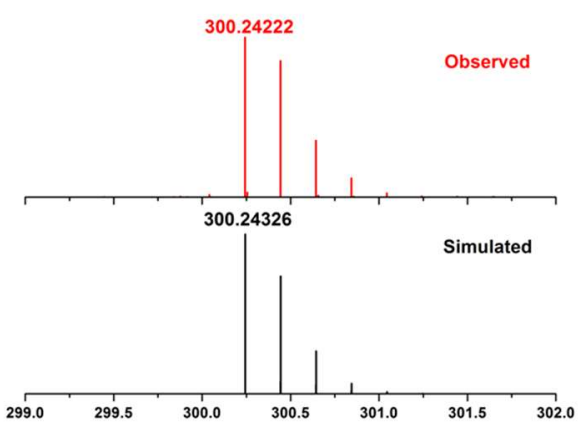

(b)

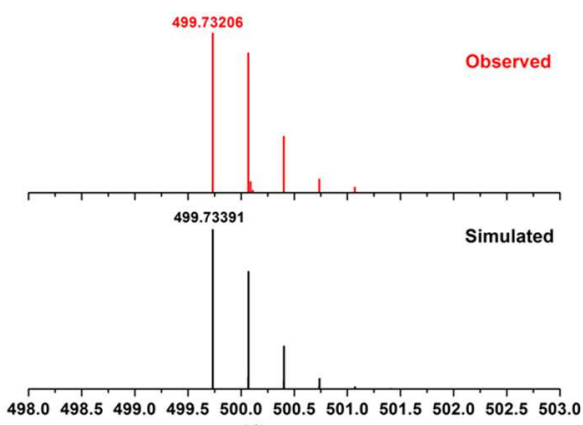

(d)

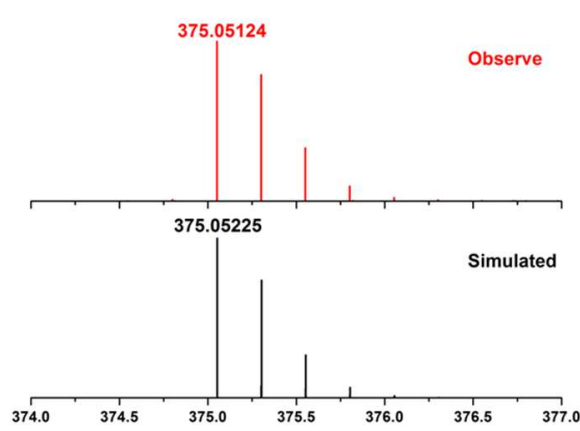

(c)

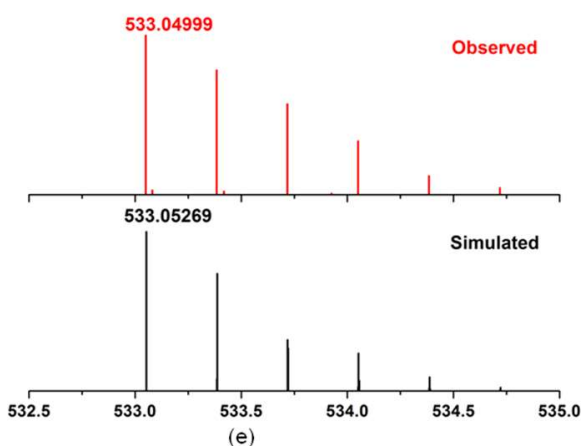

(e)

Figure S3. high resolution ESI-MS of 1, (a) full spectrum; the observed isotope patterns (red) and simulated (black) of 1: (b) $\left[\mathrm{Mn}_{4} \mathrm{C}_{68} \mathrm{H}_{57} \mathrm{O}_{8} \mathrm{~N}_{20}\right]^{5+}$, (c) $\left[\mathrm{Mn}_{4} \mathrm{C}_{68} \mathrm{H}_{56} \mathrm{O}_{8} \mathrm{~N}_{20}\right]^{4+}$, (d) $\left[\mathrm{Mn}_{4} \mathrm{C}_{68} \mathrm{H}_{55} \mathrm{O}_{8} \mathrm{~N}_{20}\right]^{3+}$, (e) $\left[\mathrm{Mn}_{4} \mathrm{C}_{67} \mathrm{H}_{56} \mathrm{O}_{8} \mathrm{~N}_{20}\left(\mathrm{ClO}_{4}\right)\right]^{3+}$. 


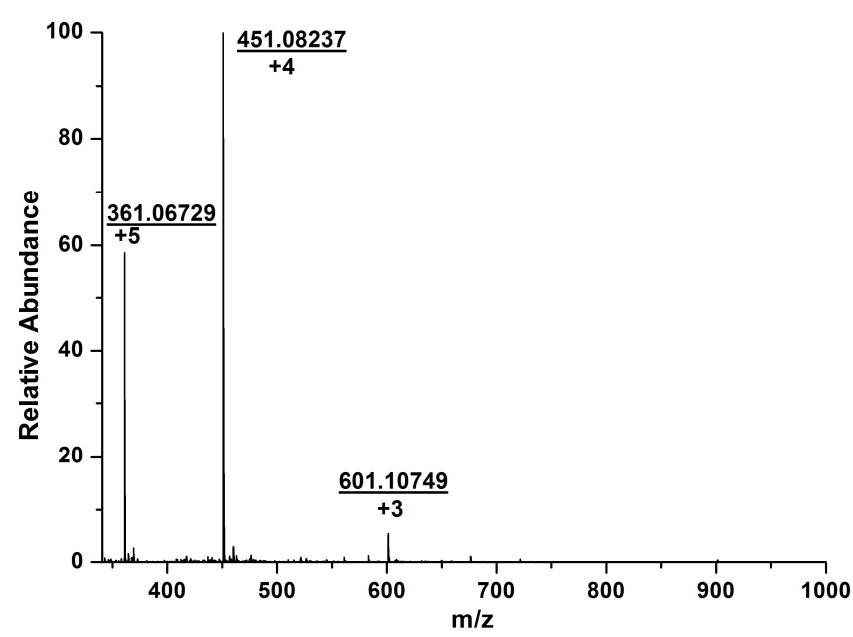

(a)
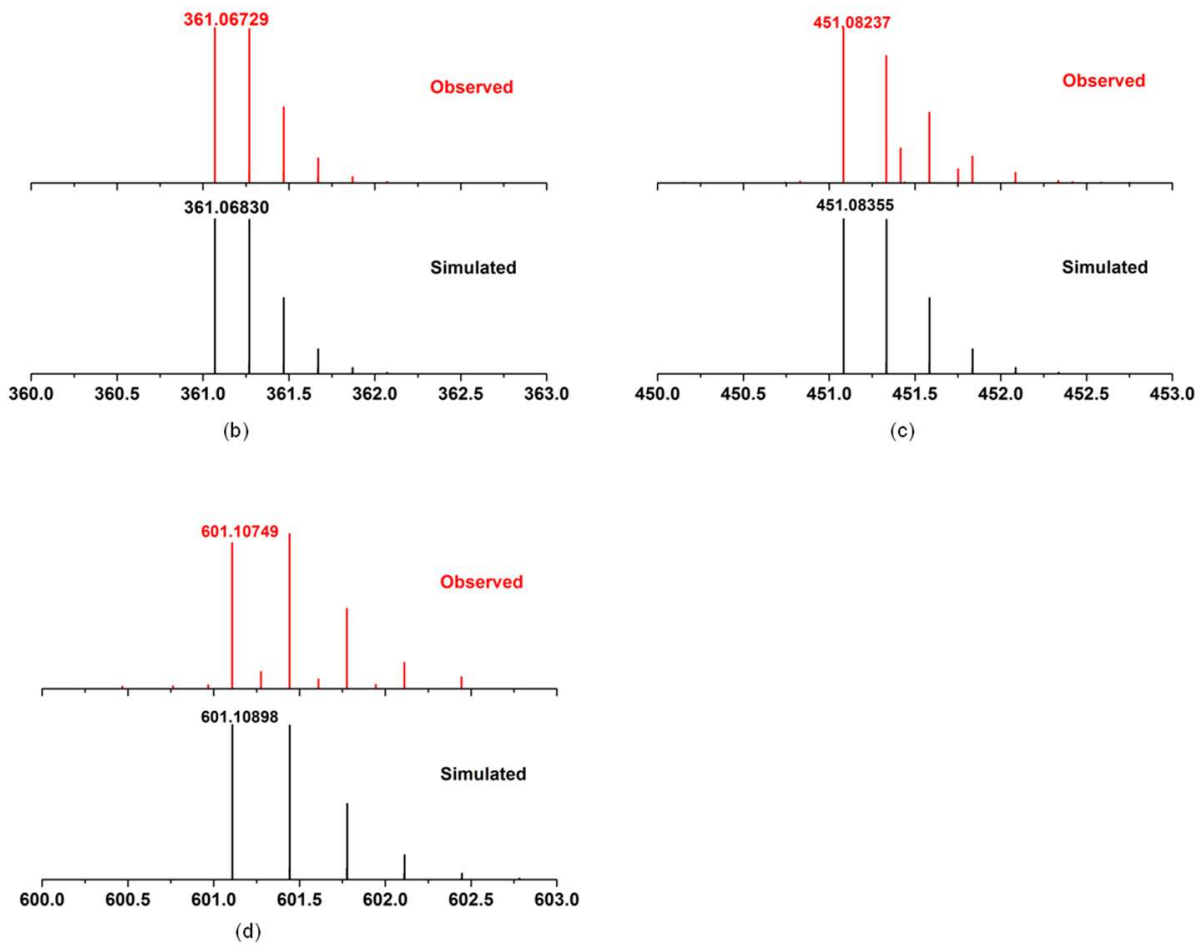

Figure S4. High resolution ESI-MS of 2, (a) full spectrum; the observed isotope patterns (red) and simulated (black) of 2: (b) $\left[\mathrm{Mn}_{4} \mathrm{C}_{92} \mathrm{H}_{73} \mathrm{O}_{8} \mathrm{~N}_{20}\right]^{5+}$, (c) $\left[\mathrm{Mn}_{4} \mathrm{C}_{92} \mathrm{H}_{72} \mathrm{O}_{8} \mathrm{~N}_{20}\right]^{4+}$, (d) $\left[\mathrm{Mn}_{4} \mathrm{C}_{92} \mathrm{H}_{71} \mathrm{O}_{8} \mathrm{~N}_{20}\right]^{3+}$. 


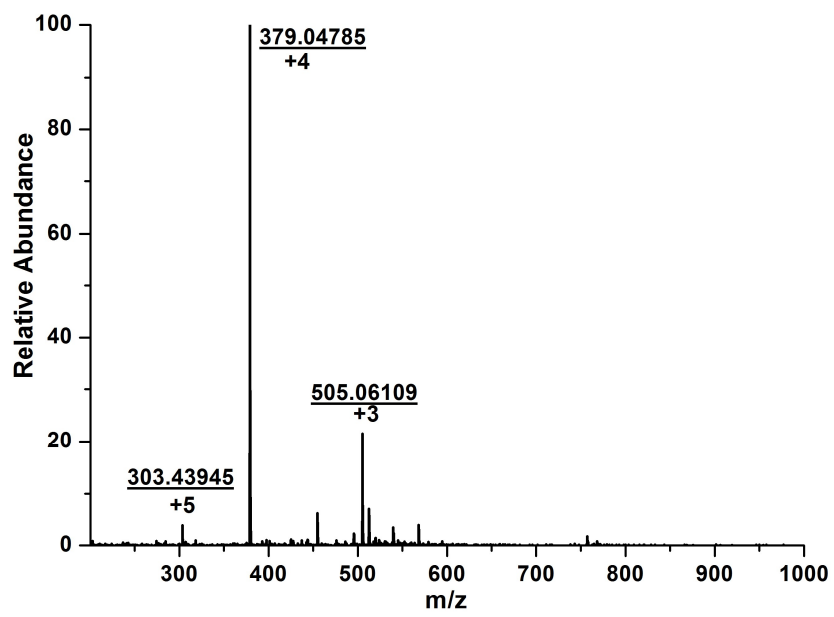

(a)

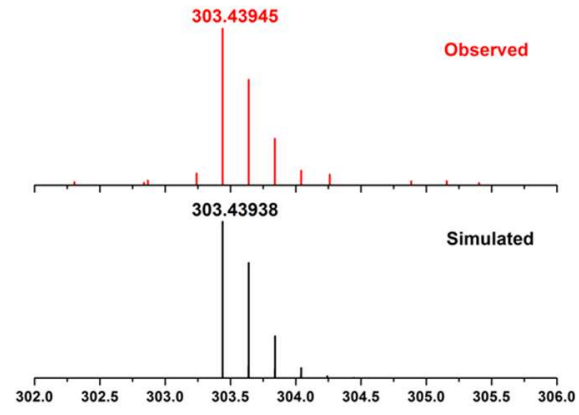

(b)

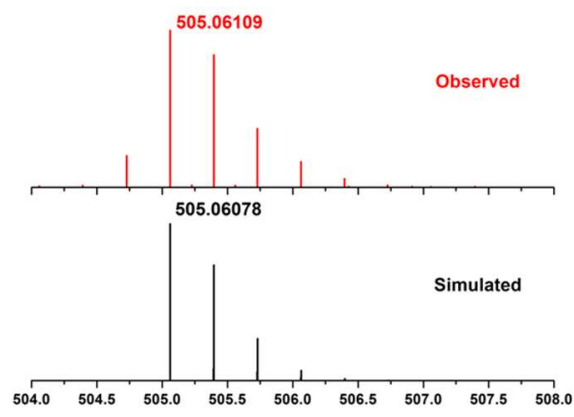

(d)

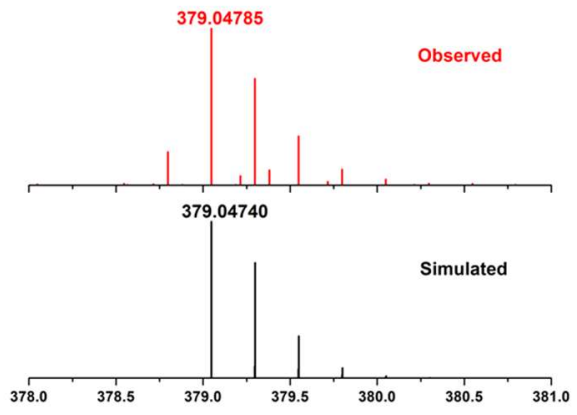

(c)

Figure S5. High resolution ESI-MS of 3, (a) full spectrum; the observed isotope patterns (red) and simulated (black) of 3: (b) $\left[\mathrm{Co}_{4} \mathrm{C}_{68} \mathrm{H}_{57} \mathrm{O}_{8} \mathrm{~N}_{20}\right]^{5+}$, (c) $\left[\mathrm{Co}_{4} \mathrm{C}_{68} \mathrm{H}_{56} \mathrm{O}_{8} \mathrm{~N}_{20}\right]^{4+}$, (d) $\left[\mathrm{Co}_{4} \mathrm{C}_{68} \mathrm{H}_{55} \mathrm{O}_{8} \mathrm{~N}_{20}\right]^{3+}$. 


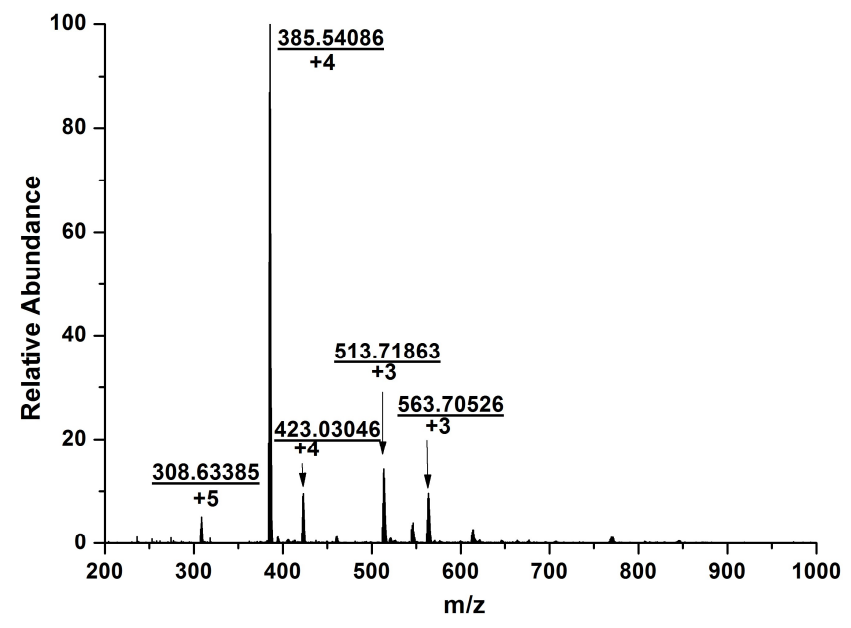

(a)

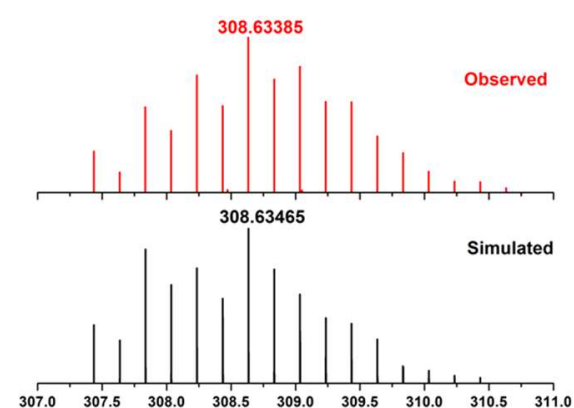

(b)

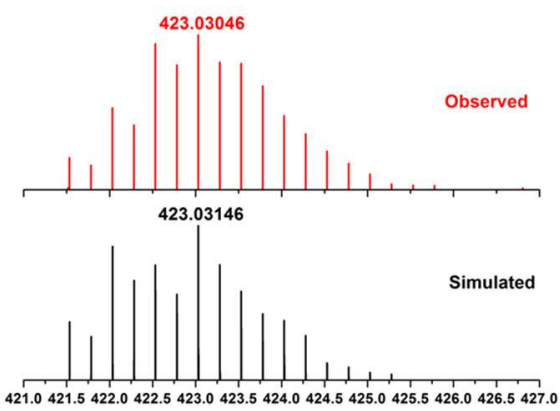

(d)

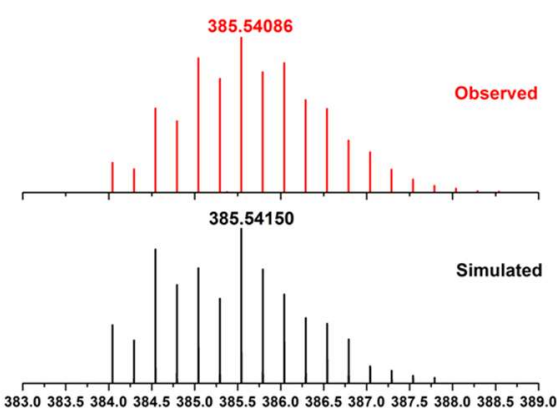

(c)

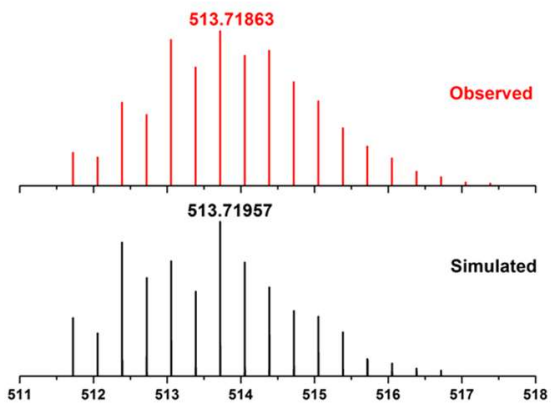

(e)

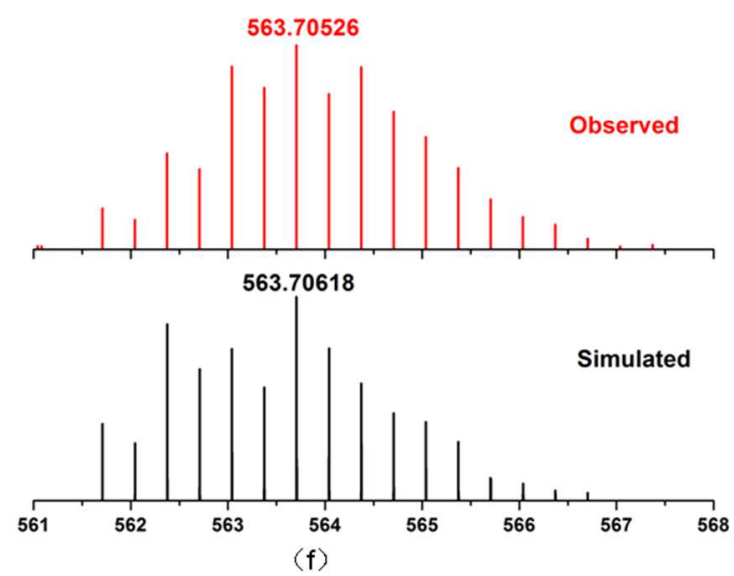

Figure S6. High resolution ESI-MS of 4, (a) full spectrum; the observed isotope patterns (red) and simulated (black) of 4: (b) $\left[\mathrm{Zn}_{4} \mathrm{C}_{68} \mathrm{H}_{57} \mathrm{O}_{8} \mathrm{~N}_{20}\right]^{5+}$, (c) $\left[\mathrm{Zn}_{4} \mathrm{C}_{68} \mathrm{H}_{56} \mathrm{O}_{8} \mathrm{~N}_{20}\right]^{4+}$, (d) $\left[\mathrm{Zn}_{4} \mathrm{C}_{68} \mathrm{H}_{57} \mathrm{O}_{8} \mathrm{~N}_{20}(\mathrm{OTf})\right]^{4+}$, (e) $\left[\mathrm{Zn}_{4} \mathrm{C}_{68} \mathrm{H}_{55} \mathrm{O}_{8} \mathrm{~N}_{20}\right]^{3+}$, (f) $\left[\mathrm{Zn}_{4} \mathrm{C}_{68} \mathrm{H}_{56} \mathrm{O}_{8} \mathrm{~N}_{20}(\mathrm{OTf})\right]^{3+}$. 


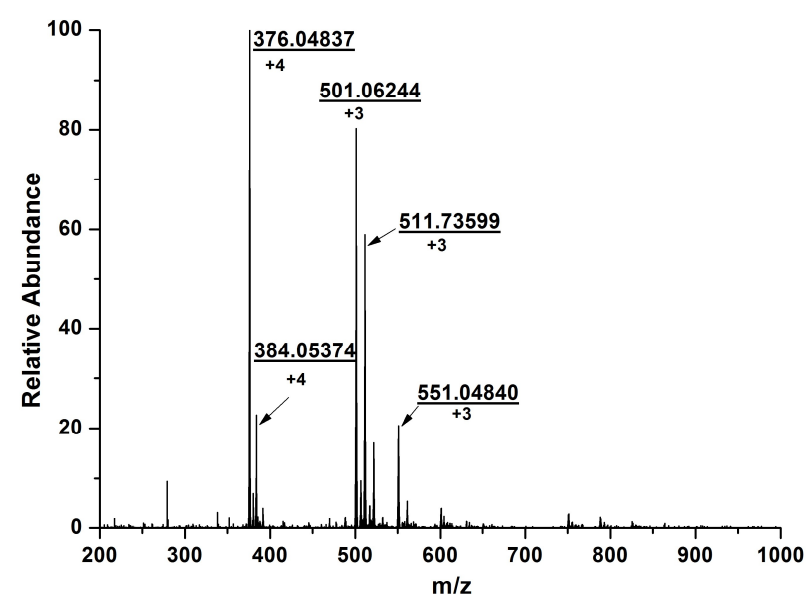

(a)

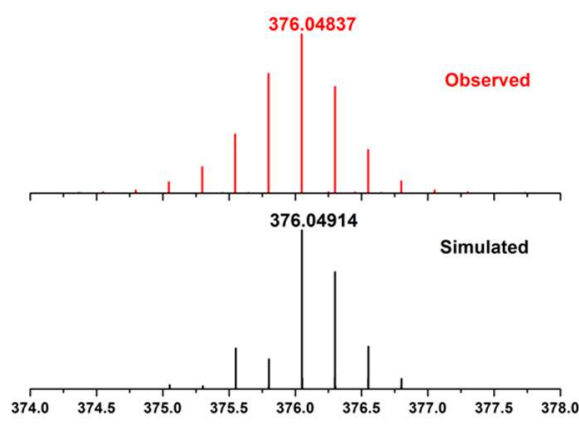

(b)

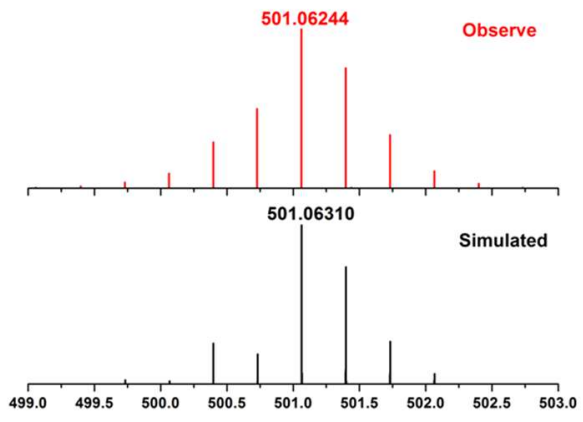

(d)

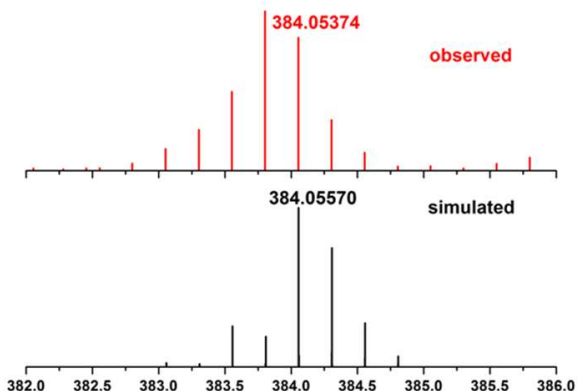

(c)

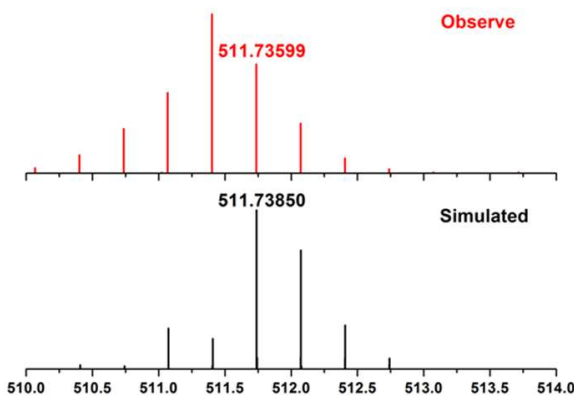

(e)

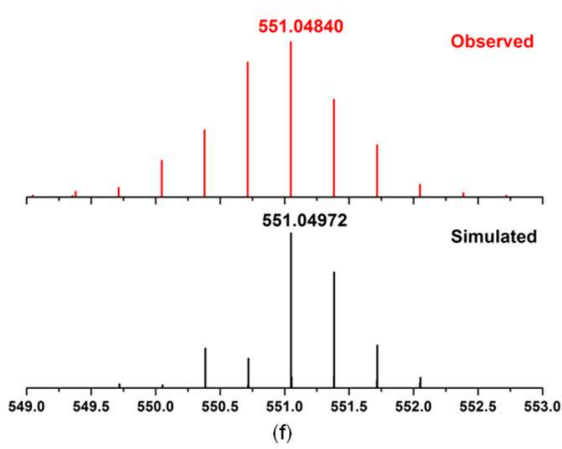

Figure S7. High resolution ESI-MS of 5, (a) full spectrum; the observed isotope patterns (red) and simulated (black) of 5: (b) $\left[\mathrm{Fe}_{4} \mathrm{C}_{68} \mathrm{H}_{56} \mathrm{O}_{8} \mathrm{~N}_{20}\right]^{4+}$, (c) $\left[\mathrm{Fe}_{4} \mathrm{C}_{68} \mathrm{H}_{56} \mathrm{O}_{8} \mathrm{~N}_{20}\left(\mathrm{CH}_{3} \mathrm{OH}\right)\right]^{4+}$, (d) $\left[\mathrm{Fe}_{4} \mathrm{C}_{68} \mathrm{H}_{55} \mathrm{O}_{8} \mathrm{~N}_{20}\right]^{3+}$, (e) $\left[\mathrm{Fe}_{4} \mathrm{C}_{68} \mathrm{H}_{55} \mathrm{O}_{8} \mathrm{~N}_{20}\left(\mathrm{CH}_{3} \mathrm{OH}\right)\right]^{3+}$, (f) $\left[\mathrm{Fe}_{4} \mathrm{C}_{68} \mathrm{H}_{56} \mathrm{O}_{8} \mathrm{~N}_{20}(\mathrm{OTf})\right]^{3+}$. 


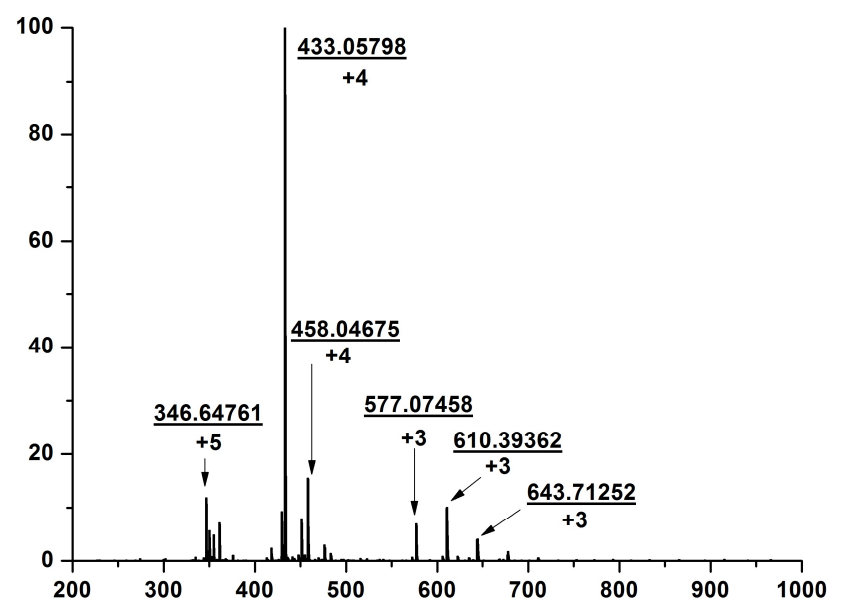

(a)

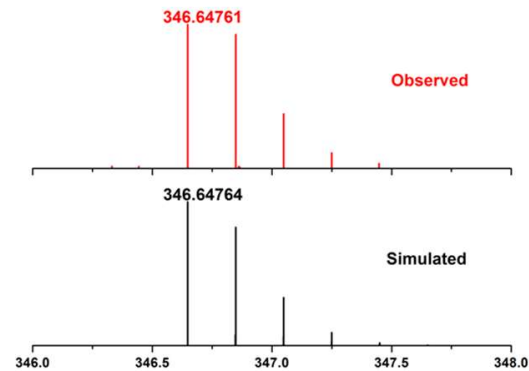

(b)
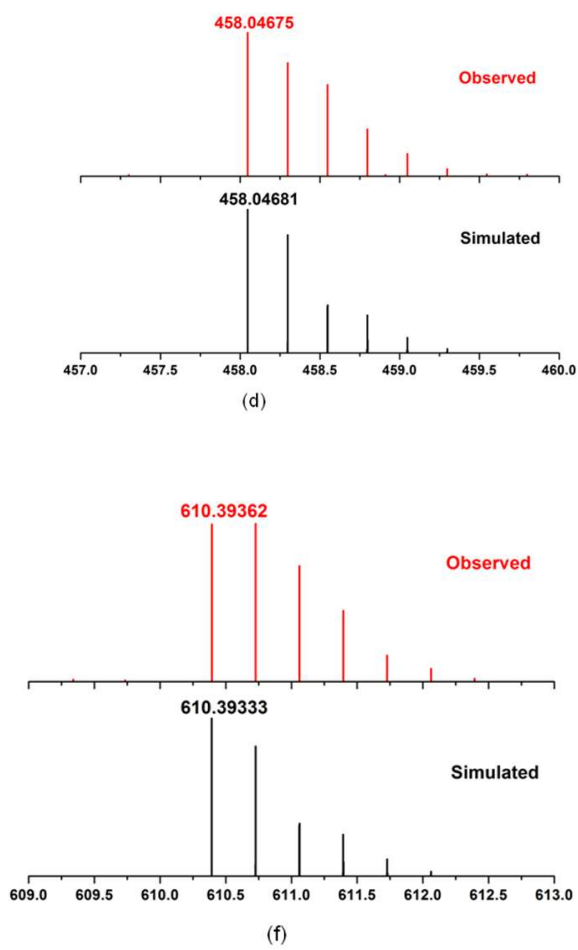

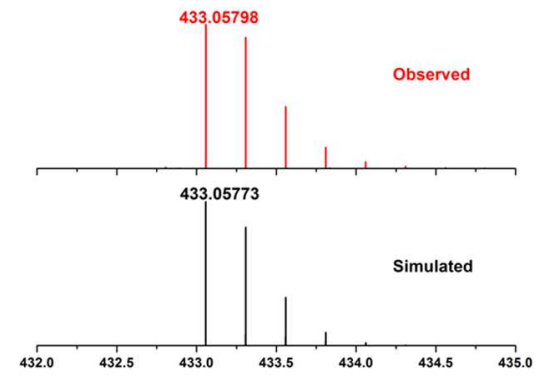

(c)
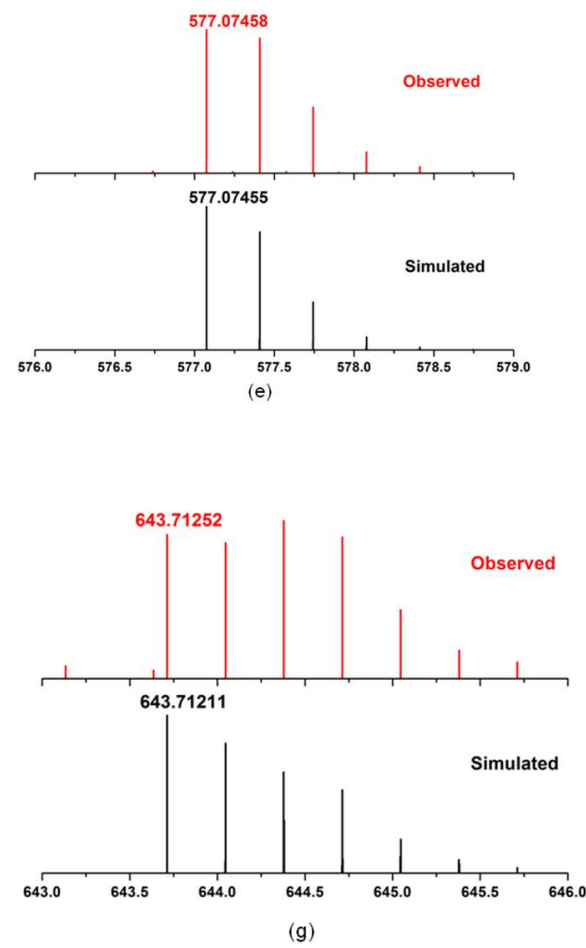

Figure S8. High resolution ESI-MS of 6, (a) full spectrum; the observed isotope patterns (red) and simulated (black) of 6 : (b) $\left[\mathrm{Mn}_{4} \mathrm{C}_{76} \mathrm{H}_{65} \mathrm{O}_{16} \mathrm{~N}_{20}\right]^{5+}, \quad$ (c) $\left[\mathrm{Mn}_{4} \mathrm{C}_{76} \mathrm{H}_{64} \mathrm{O}_{16} \mathrm{~N}_{20}\right]^{4+}$, (d) $\left[\mathrm{Mn}_{4} \mathrm{C}_{76} \mathrm{H}_{65} \mathrm{O}_{16} \mathrm{~N}_{20}\left(\mathrm{ClO}_{4}\right)\right]^{4+}, \quad$ (e) $\left[\mathrm{Mn}_{4} \mathrm{C}_{76} \mathrm{H}_{63} \mathrm{O}_{16} \mathrm{~N}_{20}\right]^{3+}, \quad$ (f) $\quad\left[\mathrm{Mn}_{4} \mathrm{C}_{76} \mathrm{H}_{64} \mathrm{O}_{16} \mathrm{~N}_{20}\left(\mathrm{ClO}_{4}\right)\right]^{3+}, \quad$ (g) $\left[\mathrm{Mn}_{4} \mathrm{C}_{76} \mathrm{H}_{65} \mathrm{O}_{16} \mathrm{~N}_{20}\left(\mathrm{ClO}_{4}\right)_{2}\right]^{3+}$. 


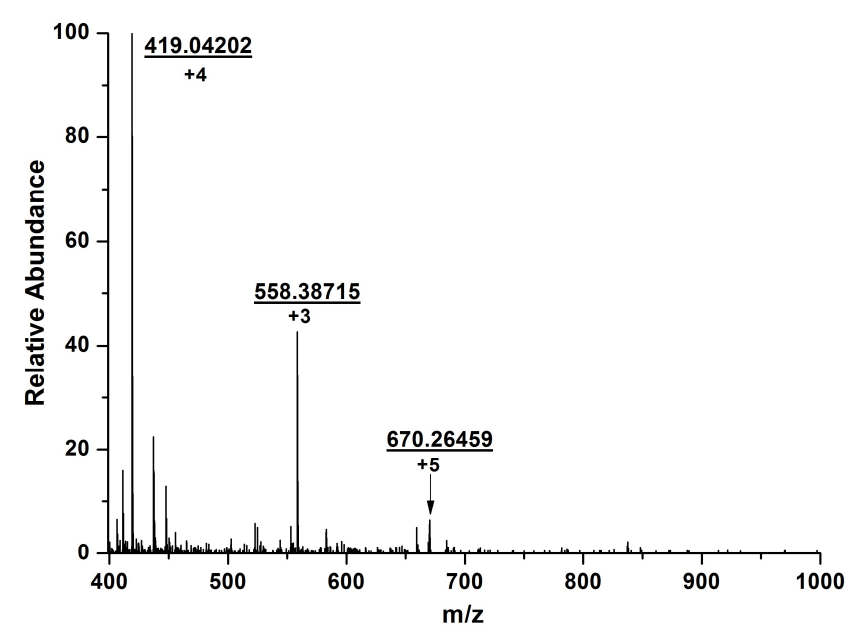

(a)

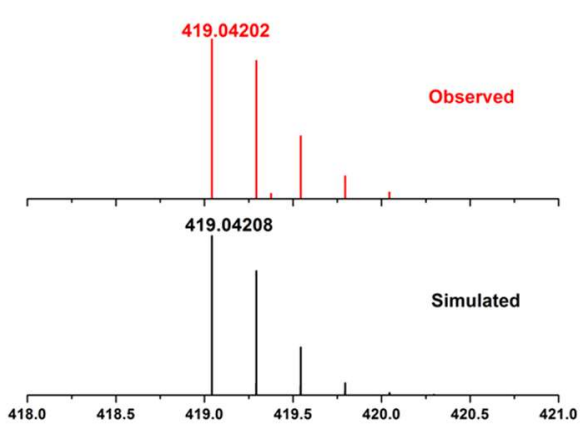

(b)
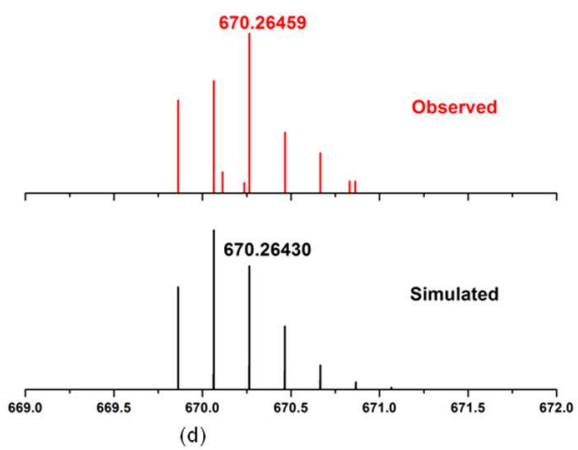

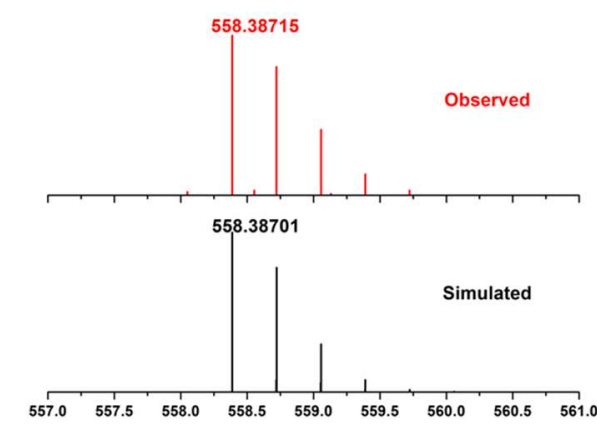

(c)

Figure S9. High resolution ESI-MS of 7, (a) full spectrum; the observed isotope patterns (red) and simulated (black) of 7: (b) $\left[\mathrm{Mn}_{4} \mathrm{C}_{72} \mathrm{H}_{56} \mathrm{O}_{16} \mathrm{~N}_{20}\right]^{4+}$, (c) $\left[\mathrm{Mn}_{4} \mathrm{C}_{72} \mathrm{H}_{55} \mathrm{O}_{16} \mathrm{~N}_{20}\right]^{3+}$, (d) $\left[\mathrm{Mn}_{8} \mathrm{C}_{144} \mathrm{H}_{109} \mathrm{O}_{32} \mathrm{~N}_{40}\right]^{5+}$. 


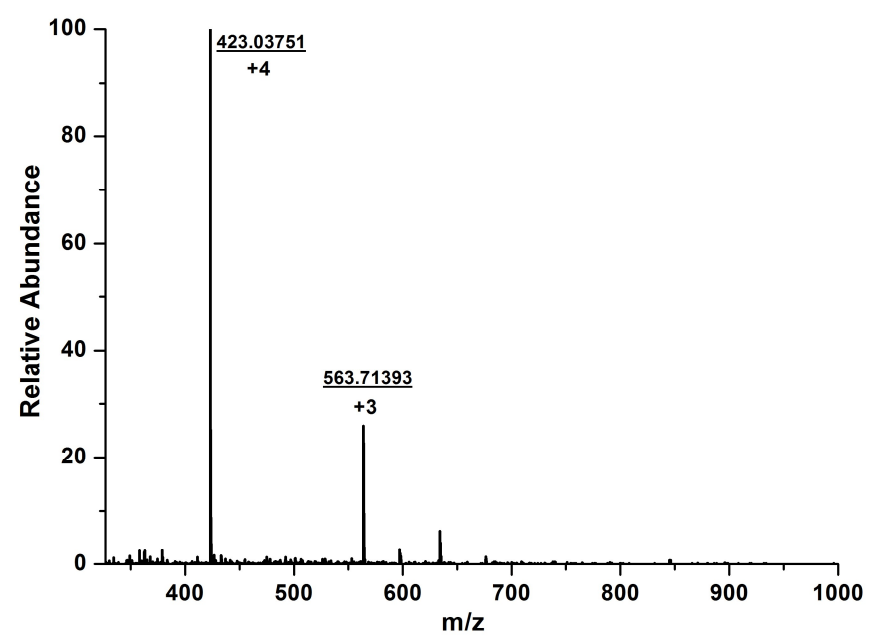

(a)

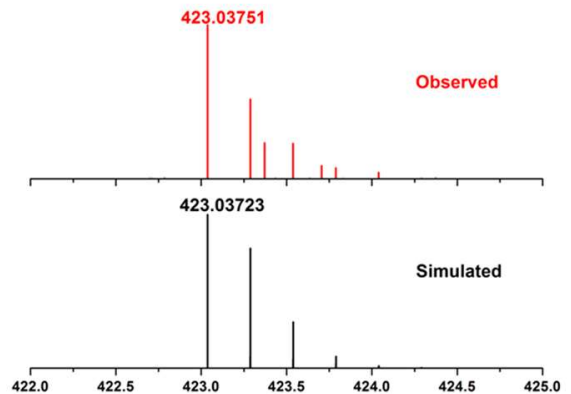

(b)

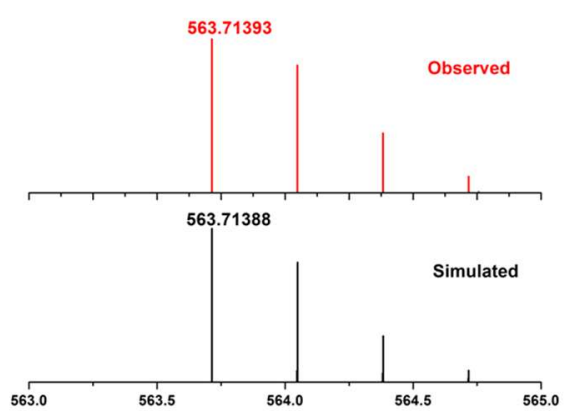

(c)

Figure S10. High resolution ESI-MS of 8, (a) full spectrum; the observed isotope patterns (red) and simulated (black) of 8: (b) $\left[\mathrm{Co}_{4} \mathrm{C}_{72} \mathrm{H}_{56} \mathrm{O}_{16} \mathrm{~N}_{20}\right]^{4+}$, (c) $\left[\mathrm{Co}_{4} \mathrm{C}_{72} \mathrm{H}_{55} \mathrm{O}_{16} \mathrm{~N}_{20}\right]^{3+}$.

6. NMR analysis of metallacycles

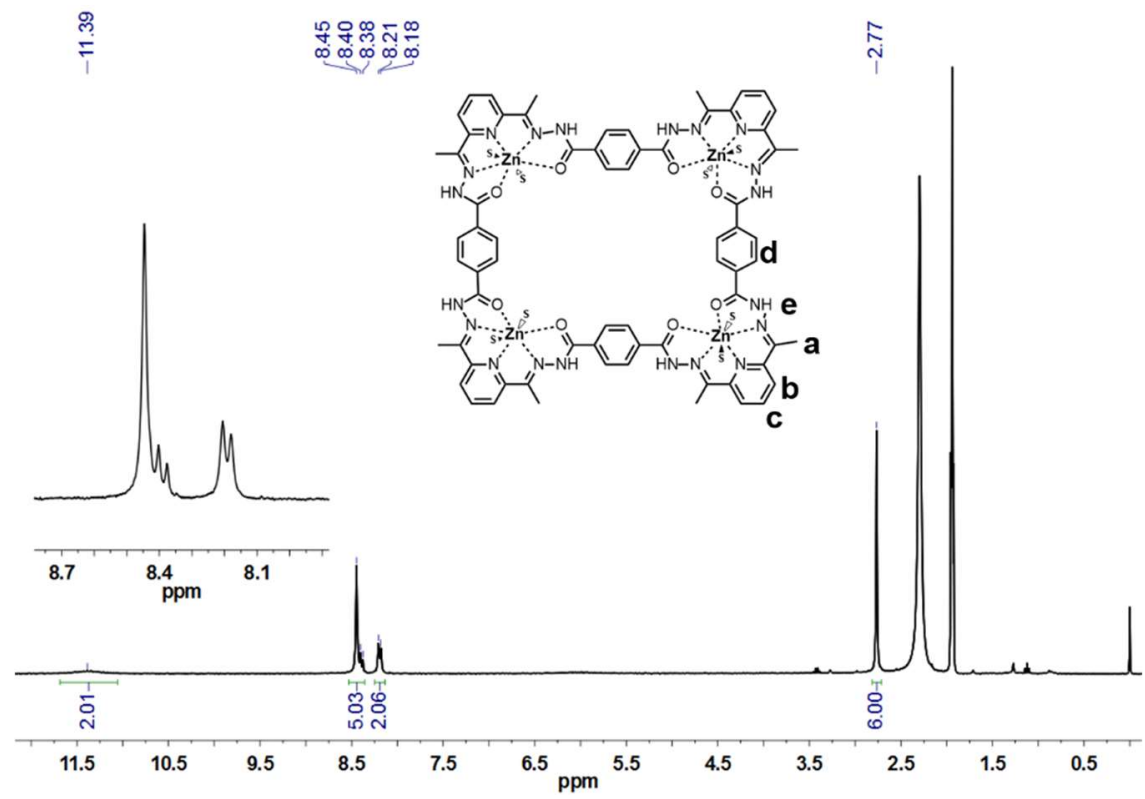

Figure S11. ${ }^{1} \mathrm{H}$ NMR spectrum and assignment of $4\left(300 \mathrm{MHz}, \mathrm{CD}_{3} \mathrm{CN}, 298 \mathrm{~K}\right)$. 


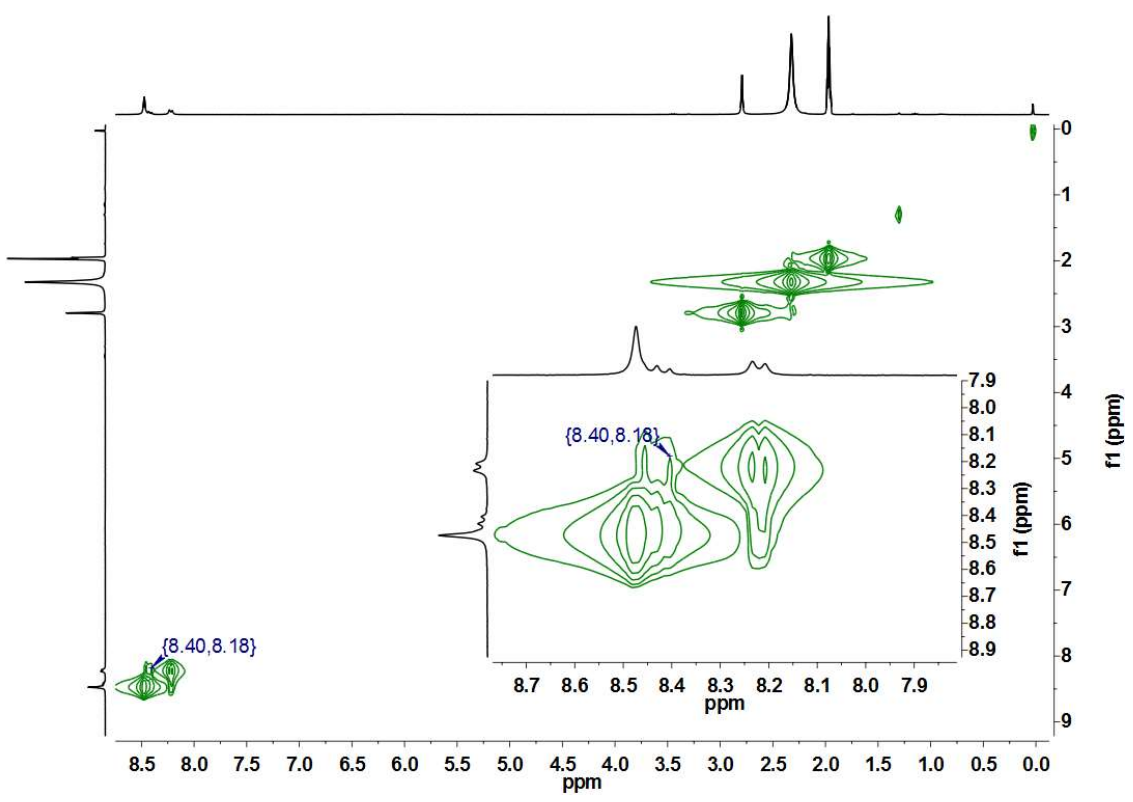

Figure S12. ${ }^{1} \mathrm{H}-{ }^{1} \mathrm{H}$ COSY spectrum of 4 (300 MHz, $\left.\mathrm{CD}_{3} \mathrm{CN}, 298 \mathrm{~K}\right)$.

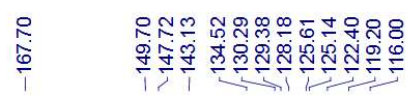

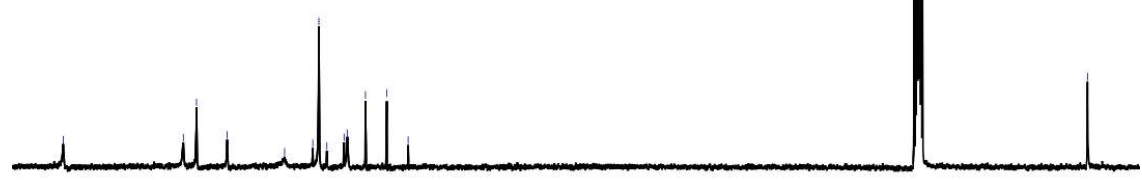

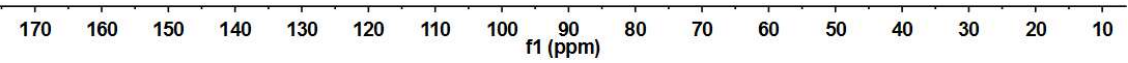

Figure S13. ${ }^{13} \mathrm{C}$ NMR spectrum of 4 (101 MHz, DMSO-d 6 , $\left.298 \mathrm{~K}\right)$. 


\section{Crystallographic data results}

Table S1. Crystallographic data of 1-3.

\begin{tabular}{|c|c|c|c|}
\hline & $1^{\prime}$ & 2 , & 3 \\
\hline CCDC number & 1911744 & 1911745 & 1911747 \\
\hline Empirical formula & $\mathrm{C}_{82} \mathrm{H}_{108} \mathrm{Mn}_{4} \mathrm{~N}_{20} \mathrm{O}_{22}$ & $\mathrm{C}_{166} \mathrm{H}_{174} \mathrm{Mn}_{4} \mathrm{~N}_{38} \mathrm{O}_{16}$ & $\mathrm{C}_{90} \mathrm{H}_{92} \mathrm{Cl}_{6} \mathrm{Co}_{4} \mathrm{~N}_{24} \mathrm{O}_{38}$ \\
\hline Formula weight & 1945.64 & 3177.18 & 2558.23 \\
\hline Temperature/K & 150.15 & $120(0)$ & $150.00(10)$ \\
\hline Crystal system & monoclinic & monoclinic & monoclinic \\
\hline Space group & $\mathrm{P} 2{ }_{1} / \mathrm{n}$ & $\mathrm{P} 2{ }_{1} / \mathrm{n}$ & $\mathrm{C} 2 / \mathrm{c}$ \\
\hline $\mathrm{a} / \AA$ & $7.66791(14)$ & $14.5195(7)$ & $47.019(2)$ \\
\hline $\mathrm{b} / \AA$ & $35.5294(6)$ & $47.357(2)$ & $8.0598(3)$ \\
\hline $\mathrm{c} / \AA ̊$ & $18.9331(3)$ & $14.8983(7)$ & $41.8518(19)$ \\
\hline$\alpha /^{\circ}$ & 90.00 & 90.00 & 90 \\
\hline$\beta /{ }^{\circ}$ & $90.5243(17)$ & $101.3080(10)$ & $123.828(4)$ \\
\hline$\gamma /{ }^{\circ}$ & 90.00 & 90 & 90 \\
\hline Volume $/ \AA^{3}$ & $5157.84(16)$ & $10045.3(8)$ & $13175.2(11)$ \\
\hline Z & 2 & 2 & 4 \\
\hline$\rho_{\text {calc }} \mathrm{g} / \mathrm{cm}^{3}$ & 1.253 & 1.050 & 1.290 \\
\hline$\mu / \mathrm{mm}^{-1}$ & 4.498 & 0.306 & 5.674 \\
\hline $\mathrm{F}(000)$ & 2032.0 & 3328.0 & 5224.0 \\
\hline Crystal size $/ \mathrm{mm}^{3}$ & $0.55 \times 0.32 \times 0.3$ & $0.161 \times 0.136 \times 0.120$ & $\begin{array}{c}0.150 \times 0.050 \times \\
0.040\end{array}$ \\
\hline Radiation & $\operatorname{CuK} \alpha(\lambda=1.54184)$ & $\operatorname{MoK} \alpha(\lambda=0.71073)$ & $\operatorname{CuK} \alpha(\lambda=1.54178)$ \\
\hline $\begin{array}{l}2 \Theta \text { range for data } \\
\text { collection } /{ }^{\circ}\end{array}$ & 8.8 to 148.2 & 4.414 to 50.848 & 7.52 to 130.968 \\
\hline Index ranges & $\begin{array}{l}-9 \leq \mathrm{h} \leq 6,-43 \leq \mathrm{k} \leq \\
42 \\
-22 \leq 1 \leq 23\end{array}$ & $\begin{array}{l}-17 \leq \mathrm{h} \leq 16,-56 \leq \mathrm{k} \leq \\
57,-14 \leq 1 \leq 17\end{array}$ & $\begin{array}{l}-40 \leq \mathrm{h} \leq 54,-6 \leq \mathrm{k} \leq \\
9 \\
-48 \leq 1 \leq 46\end{array}$ \\
\hline Reflections collected & 22060 & 81002 & 34165 \\
\hline Independent reflections & $\begin{array}{c}10194\left[\mathrm{R}_{\text {int }}=\right. \\
0.0329 \\
\left.\mathrm{R}_{\text {sigma }}=0.0410\right]\end{array}$ & $\begin{array}{c}18260\left[\mathrm{R}_{\text {int }}=0.0406,\right. \\
\left.\quad \mathrm{R}_{\text {sigma }}=0.0465\right]\end{array}$ & $\begin{array}{c}11078\left[\mathrm{R}_{\text {int }}=0.0737,\right. \\
\left.\mathrm{R}_{\text {sigma }}=0.1003\right]\end{array}$ \\
\hline Data/restraints/parameters & $10194 / 0 / 582$ & $18260 / 63 / 998$ & $11078 / 43 / 684$ \\
\hline Goodness-of-fit on $\mathrm{F}^{2}$ & 1.048 & 1.065 & 1.228 \\
\hline Final $R$ indexes $[\mathrm{I}>=2 \sigma$ & $\mathrm{R}_{1}=0.0525, \mathrm{wR}_{2}=$ & $\mathrm{R}_{1}=0.0793, \mathrm{wR}_{2}=$ & $\mathrm{R}_{1}=0.1293, \mathrm{wR}_{2}=$ \\
\hline (I)] & 0.1446 & 0.2406 & 0.3523 \\
\hline Final $\mathrm{R}$ indexes [all data] & $\begin{array}{l}\mathrm{R}_{1}=0.0573, \mathrm{wR}_{2}= \\
0.1488\end{array}$ & $\begin{array}{l}\mathrm{R}_{1}=0.1036, \mathrm{wR}_{2}= \\
0.2541\end{array}$ & $\begin{array}{l}\mathrm{R}_{1}=0.1740, \mathrm{wR}_{2}= \\
0.3894\end{array}$ \\
\hline $\begin{array}{l}\text { Largest diff. peak/hole / } \\
\mathrm{e}^{-} \AA^{-3}\end{array}$ & $1.86 /-0.57$ & $0.63 /-0.71$ & $1.14 /-1.05$ \\
\hline
\end{tabular}


Table S2. Crystallographic data of 4 and 6.

\begin{tabular}{|c|c|c|}
\hline & 4 & $6^{\prime}$ \\
\hline CCDC number & 1970657 & 1911746 \\
\hline Empirical formula & $\mathrm{C}_{37} \mathrm{H}_{34} \mathrm{~F}_{3} \mathrm{~N}_{10} \mathrm{O}_{10} \mathrm{SZn}_{2}$ & $\mathrm{C}_{136} \mathrm{H}_{94} \mathrm{Mn}_{4} \mathrm{~N}_{32} \mathrm{O}_{18}$ \\
\hline Formula weight & 998.54 & 2684.19 \\
\hline Temperature/K & 119.99 & 120.02 \\
\hline Crystal system & monoclinic & triclinic \\
\hline Space group & $\mathrm{P} 21 / \mathrm{m}$ & P-1 \\
\hline $\mathrm{a} / \AA$ & $14.8683(17)$ & $10.8346(4)$ \\
\hline $\mathrm{b} / \AA ̊$ & $35.964(4)$ & $16.7939(6)$ \\
\hline $\mathrm{c} / \AA$ & $15.7673(18)$ & $19.7458(7)$ \\
\hline$\alpha /{ }^{\circ}$ & 90 & $107.577(2)$ \\
\hline$\beta /{ }^{\circ}$ & $115.880(3)$ & $92.476(2)$ \\
\hline$\gamma /{ }^{\circ}$ & 90 & $97.574(2)$ \\
\hline Volume $/ \AA^{3}$ & $7585.6(15)$ & $3382.1(2)$ \\
\hline Z & 4 & 1 \\
\hline$\rho_{\text {calc }} \mathrm{g} / \mathrm{cm}^{3}$ & 0.874 & 1.318 \\
\hline$\mu / \mathrm{mm}^{-1}$ & 0.705 & 0.441 \\
\hline $\mathrm{F}(000)$ & 2036.0 & 1378.0 \\
\hline Crystal size $/ \mathrm{mm}^{3}$ & $0.205 \times 0.184 \times 0.15$ & $0.113 \times 0.059 \times 0.043$ \\
\hline Radiation & $\operatorname{MoK} \alpha(\lambda=0.71073)$ & $\operatorname{MoK} \alpha(\lambda=0.71073)$ \\
\hline $2 \Theta$ range for data collection $/^{\circ}$ & 3.044 to 55.178 & 4.344 to 55.162 \\
\hline Index ranges & $\begin{array}{c}-16 \leq \mathrm{h} \leq 19,-46 \leq \mathrm{k} \leq 46 \\
-20 \leq 1 \leq 16\end{array}$ & $\begin{array}{c}-14 \leq \mathrm{h} \leq 14,-21 \leq \mathrm{k} \leq 21 \\
-25 \leq 1 \leq 25\end{array}$ \\
\hline Reflections collected & 62698 & 96194 \\
\hline Independent reflections & $\begin{array}{c}17661\left[\mathrm{R}_{\text {int }}=0.1014,\right. \\
\left.\mathrm{R}_{\text {sigma }}=0.1533\right]\end{array}$ & $\begin{array}{c}15567\left[\mathrm{R}_{\text {int }}=0.0934,\right. \\
\left.\mathrm{R}_{\text {sigma }}=0.0711\right]\end{array}$ \\
\hline Data/restraints/parameters & $17661 / 152 / 540$ & $15567 / 400 / 795$ \\
\hline Goodness-of-fit on $\mathrm{F}^{2}$ & 1.329 & 1.094 \\
\hline Final $\mathrm{R}$ indexes $[\mathrm{I}>=2 \sigma(\mathrm{I})]$ & $\mathrm{R}_{1}=0.1471, \mathrm{wR}_{2}=0.4230$ & $\mathrm{R}_{1}=0.0817, \mathrm{wR}_{2}=0.2608$ \\
\hline Final $\mathrm{R}$ indexes [all data] & $\mathrm{R}_{1}=0.2173, \mathrm{wR}_{2}=0.4519$ & $\mathrm{R}_{1}=0.1260, \mathrm{wR}_{2}=0.2928$ \\
\hline Largest diff. peak/hole / e $\AA^{-3}$ & $2.70 /-0.81$ & $2.44 /-1.21$ \\
\hline
\end{tabular}

$\mathbf{R}_{1}=\sum|| F_{O}|-| F_{C}|| / \sum\left|F_{O}\right| ; \mathbf{w R}_{2}=\left[\sum \mathbf{w}\left(F_{O}^{2}-F_{c}^{2}\right)^{2} / \sum \mathbf{w}\left(F_{o}^{2}\right)^{2}\right]^{1 / 2}$.

\subsection{Response to the alerts in the crystallographic data.}

Response to the alerts involved in the crystallographic data of 1' (CCDC 1911744):

PLAT420_ALERT_2_B D-H Without Acceptor O7 --H7. Please Check

PLAT420_ALERT_2_B D-H Without Acceptor O9 --H9. Please Check

PLAT420_ALERT_2_B D-H Without Acceptor O11 --H11. Please Check

All these alerts are because of the missing of H-Bond acceptors of methanol molecules, while the acceptors of weak X-ray diffraction were found highly disordered and were squeezed by Platon. ${ }^{\text {S5 }}$

PLAT990_ALERT_1_B Deprecated .res/.hkl Input Style SQUEEZE Job ...! Note

This alert comes from the use of squeeze program of Platon software.

Response to the alerts involved in the crystallographic data of 2' (CCDC 1911745):

PLAT990_ALERT_1_B Deprecated .res/. hkl Input Style SQUEEZE Job ...! Note 
This alert comes from the use of squeeze program of Platon software. ${ }^{\mathrm{S} 5}$

Response to the alerts involved in the crystallographic data of 3 (CCDC 1911747):

PLAT084_ALERT_3_B High wR2 Value (i.e. > 0.25) …………... 0.39 Report

This alert comes from the poor quality of the crystal. Multiple attempts of crystal preparations and data collection did not succeed. This poor data quality was common in this class of metallacycles with large spaces filled with disordered anions and solvent molecules. However, the data quality doesn't affect the discussion of structure.

PLAT420_ALERT_2_B D-H Without Acceptor O3 --H3A. Please Check

PLAT420_ALERT_2_B D-H Without Acceptor O6 --H6B. Please Check

These alerts come from the missing of $\mathrm{H}$-Bond acceptors of $\mathrm{H}_{2} \mathrm{O}$ molecules that coordinated to the $\mathrm{Co}(\mathrm{II})$ ions, while the acceptors of weak X-ray diffraction were found highly disordered and were squeezed by Platon. ${ }^{\text {S5 }}$

PLAT990_ALERT_1_B Deprecated .res/. hkl Input Style SQUEEZE Job ...! Note

This alert comes from the use of squeeze program of Platon software. ${ }^{\mathrm{S} 5}$

Response to the alerts involved in the crystallographic data of 4 (CCDC 1911747):

PLAT084_ALERT_3_B High wR2 Value (i.e. > 0.25) 0.45 Report

The explanation of this alert is similar to the alert involved in the data of 3 .

PLAT420_ALERT_2_B D-H Without Acceptor O9 -H9B. Please Check

This alert comes from the missing of $\mathrm{H}-\mathrm{B}$-nd acceptors of $\mathrm{H}_{2} \mathrm{O}$ molecules that coordinated to the $\mathrm{Zn}$ (II) ions, while the acceptors of weak X-ray diffraction were found highly disordered and were squeezed by Platon. s5 $^{2}$ 
(a)

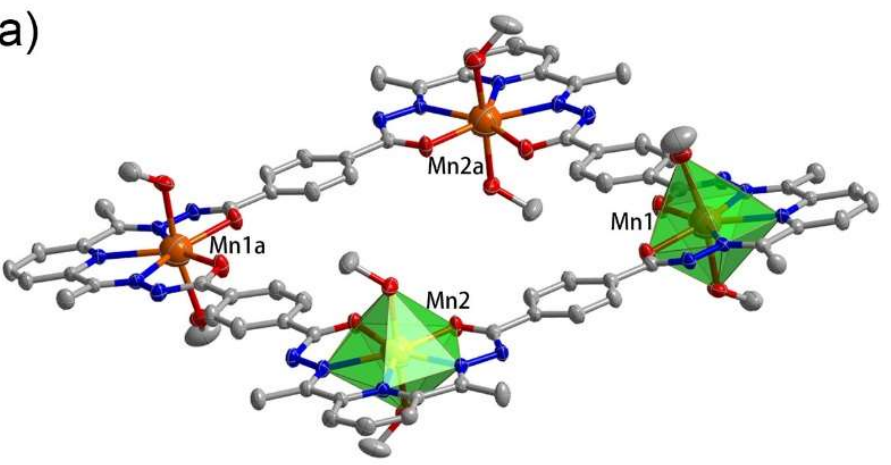

(b)

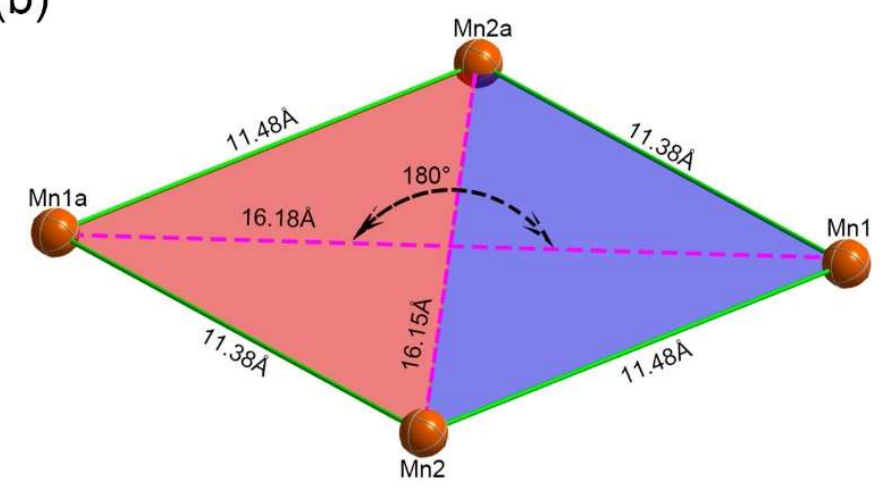

Figure S14. (a) ORTEP drawing of 1' with $60 \%$ thermal ellipsoids. All hydrogen atoms and solvents were omitted for clarity (C: gray, N: blue, O: red, Mn: orange). Pentagonal bipyramidal coordination spheres are highlighted in green. (b) Simplified structural model of $\mathbf{1}$ '.
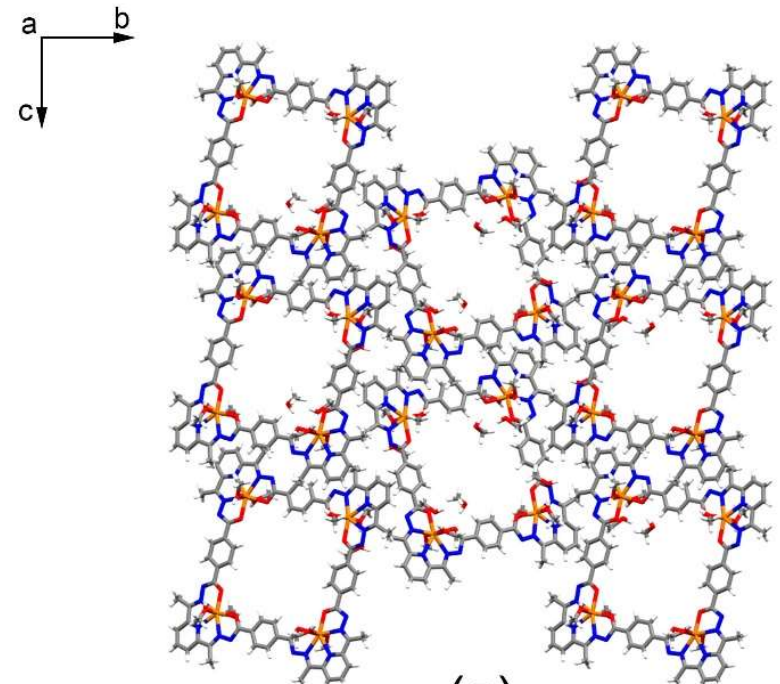

(a)
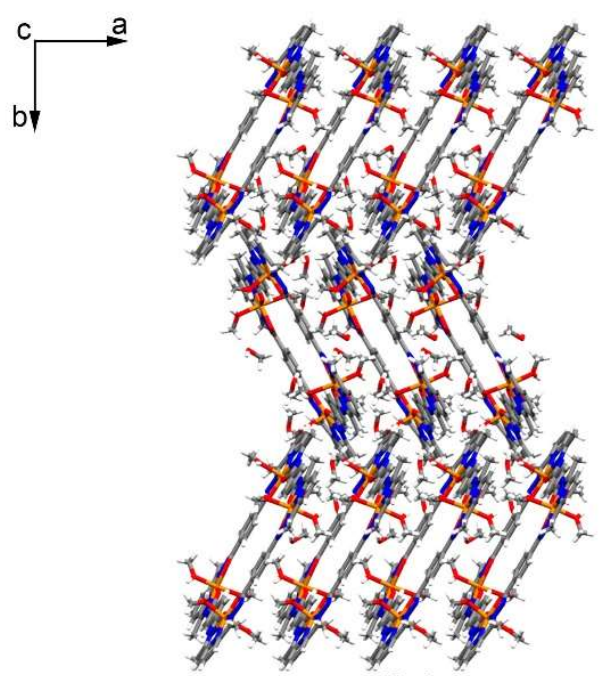

(b)

Figure S15. Packing diagram of 1' along the a-axis (a) and c-axis (b), respectively. 
Table S3. Selected Bond Distances ( $\AA$ ) and Angles (deg) for 1'.

\begin{tabular}{|c|c|c|c|}
\hline \multicolumn{4}{|c|}{ Bond lengths } \\
\hline Bond & Length $(\AA)$ & Bond & Length $(\AA)$ \\
\hline Mn1-O1 & $2.2036(18)$ & $\mathrm{Mn} 2-\mathrm{O} 5$ & $2.2219(19)$ \\
\hline $\mathrm{Mn} 1-\mathrm{O} 2$ & $2.2476(18)$ & Mn2-O6 & $2.2107(18)$ \\
\hline Mn1-O3 & $2.2270(19)$ & $\mathrm{Mn2-O7}$ & $2.258(2)$ \\
\hline Mn1-O4 & $2.2426(19)$ & $\mathrm{Mn} 2-\mathrm{O} 8$ & $2.2327(19)$ \\
\hline Mn1-N2 & $2.275(2)$ & Mn2-N9 & $2.318(2)$ \\
\hline Mn1-N3 & $2.329(2)$ & Mn2-N8 & $2.342(2)$ \\
\hline Mn1-N4 & $2.289(2)$ & Mn2-N7 & $2.282(2)$ \\
\hline \multicolumn{4}{|c|}{ Bond angle } \\
\hline Bond angle & Degree $\left(^{\circ}\right)$ & Bond angle & Degree $\left(^{\circ}\right)$ \\
\hline O1-Mn1-N4 & $69.56(7)$ & O5-Mn2-N7 & $69.42(7)$ \\
\hline $\mathrm{O} 2-\mathrm{Mn} 1-\mathrm{N} 2$ & $69.10(7)$ & O5-Mn2-O6 & $87.75(7)$ \\
\hline $\mathrm{O} 1-\mathrm{Mn} 1-\mathrm{O} 2$ & $86.27(7)$ & O6-Mn2-N9 & $68.63(7)$ \\
\hline N2-Mn1-N3 & $67.55(7)$ & O7-Mn2-O8 & $168.86(8)$ \\
\hline N4-Mn1-N3 & $67.68(7)$ & N7-Mn2-N8 & $67.70(7)$ \\
\hline O3-Mn1-O4 & $171.56(8)$ & N8-Mn2-N9 & $66.99(7)$ \\
\hline
\end{tabular}




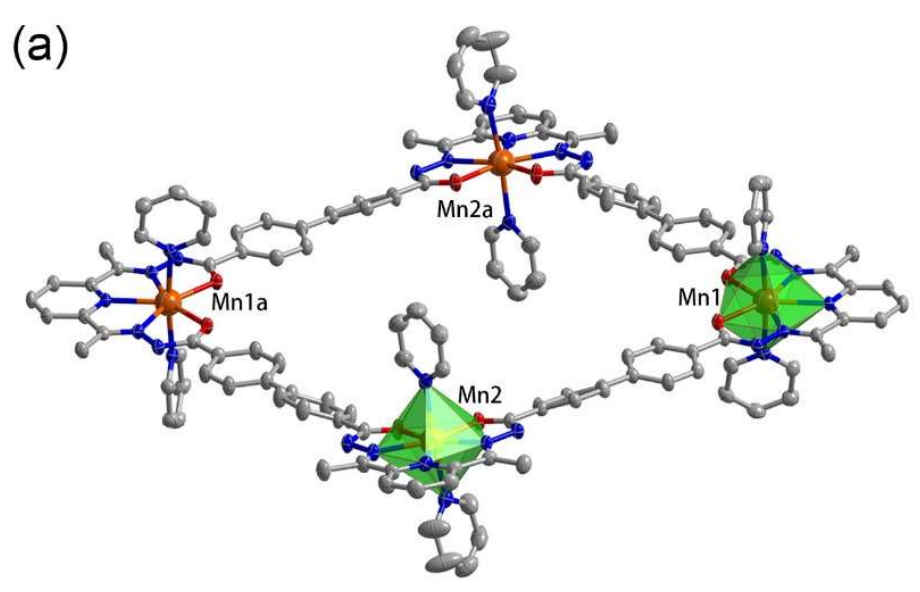

(b)

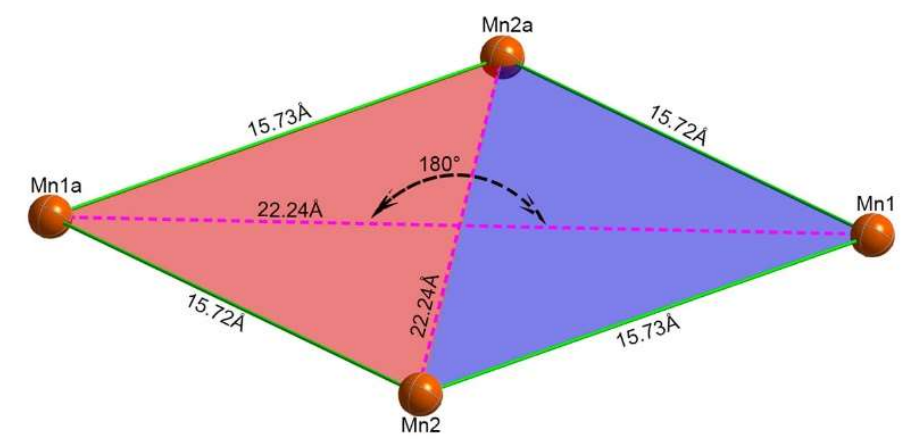

Figure S16. (a) ORTEP drawing of 2' with $40 \%$ thermal ellipsoids. All hydrogen atoms and solvents were omitted for clarity (C: gray, N: blue, O: red, Mn: orange). Pentagonal bipyramidal coordination spheres are highlighted in green. (b) Simplified structural model of 2'.
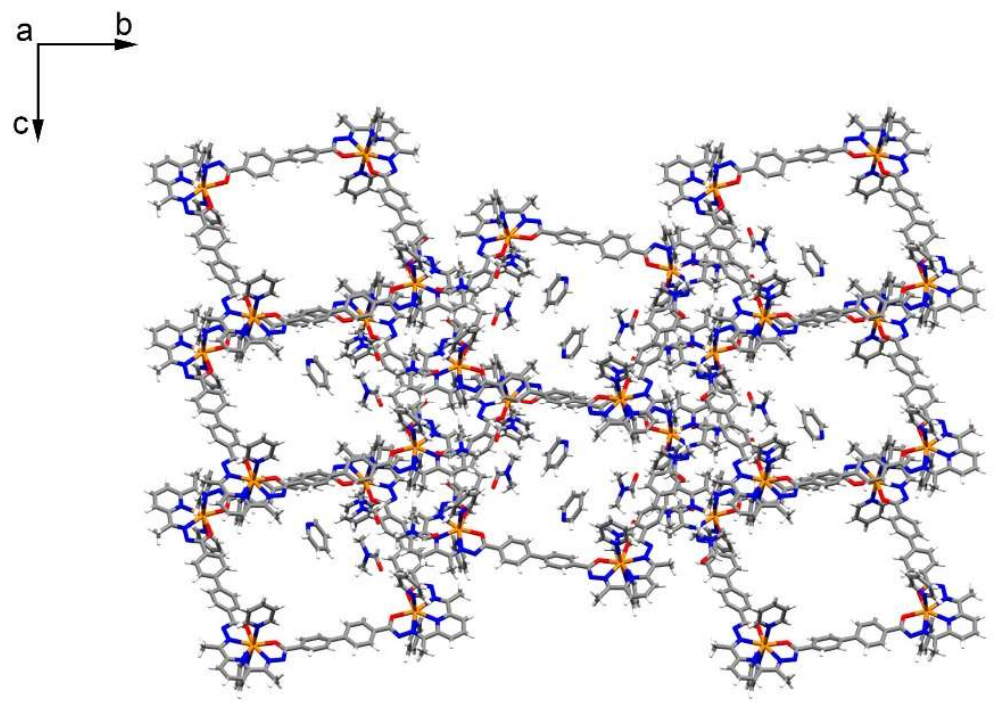

(a)
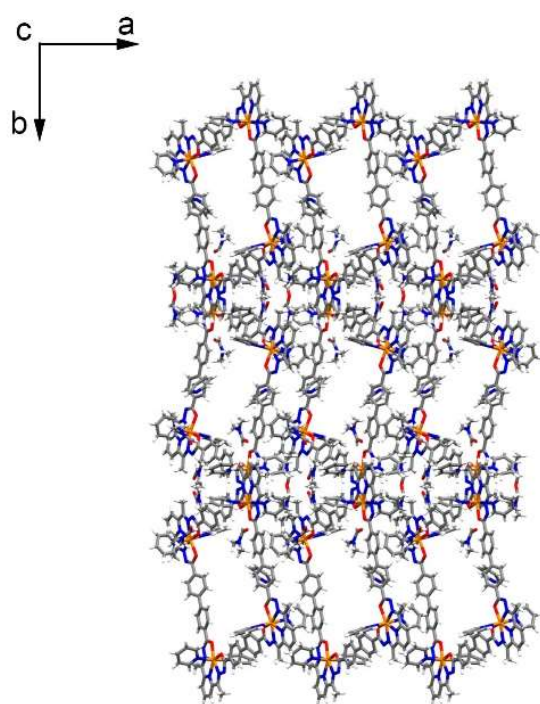

(b)

Figure S17. Packing diagram of 2' along the a-axis (a) and c-axis (b), respectively. 
Table S4. Selected Bond Distances ( $\AA$ ) and Angles (deg) for 2'.

\begin{tabular}{|c|c|c|c|}
\hline \multicolumn{4}{|c|}{ Bond lengths } \\
\hline Bond & Length $(\AA)$ & Bond & Length $(\AA)$ \\
\hline Mn1-O1 & $2.222(2)$ & Mn2-O3 & $2.232(3)$ \\
\hline Mn1-O2 & $2.225(3)$ & Mn2-O4 & $2.217(3)$ \\
\hline Mn1-N2 & $2.287(3)$ & Mn2-N11 & $2.305(3)$ \\
\hline Mn1-N3 & $2.332(3)$ & Mn2-N10 & $2.320(3)$ \\
\hline Mn1-N4 & $2.314(3)$ & Mn2-N9 & $2.289(3)$ \\
\hline Mn1-N6 & $2.281(3)$ & Mn2-N13 & $2.322(4)$ \\
\hline Mn1-N7 & $2.284(3)$ & Mn2-N14 & $2.333(4)$ \\
\hline \multicolumn{4}{|c|}{ Bond angle } \\
\hline Bond angle & Degree $\left(^{\circ}\right)$ & Bond angle & Degree $\left(^{\circ}\right)$ \\
\hline $\mathrm{O} 1-\mathrm{Mn} 1-\mathrm{O} 2$ & $86.94(9)$ & O3-Mn2-N9 & $68.98(11)$ \\
\hline $\mathrm{O} 1-\mathrm{Mn} 1-\mathrm{N} 2$ & $69.14(10)$ & $\mathrm{O} 4-\mathrm{Mn} 2-\mathrm{O} 3$ & $86.59(10)$ \\
\hline N2-Mn1-N3 & $67.56(11)$ & O4-Mn2-N11 & $69.16(10)$ \\
\hline N4-Mn1-N3 & $67.51(11)$ & N9-Mn2-N10 & $68.02(11)$ \\
\hline $\mathrm{O} 2-\mathrm{Mn} 1-\mathrm{N} 4$ & $68.87(10)$ & N11-Mn2-N10 & $67.34(11)$ \\
\hline N6-Mn1-N7 & $173.92(11)$ & N13-Mn2-N14 & $175.10(13)$ \\
\hline
\end{tabular}


(a)

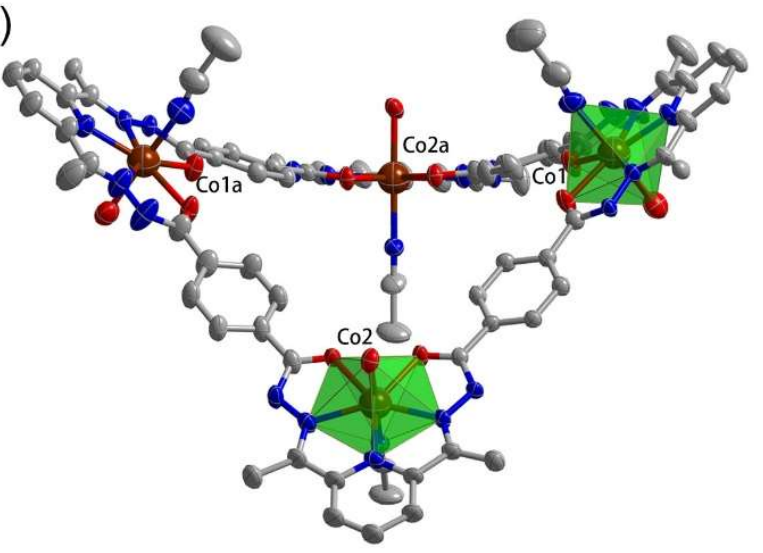

(b)

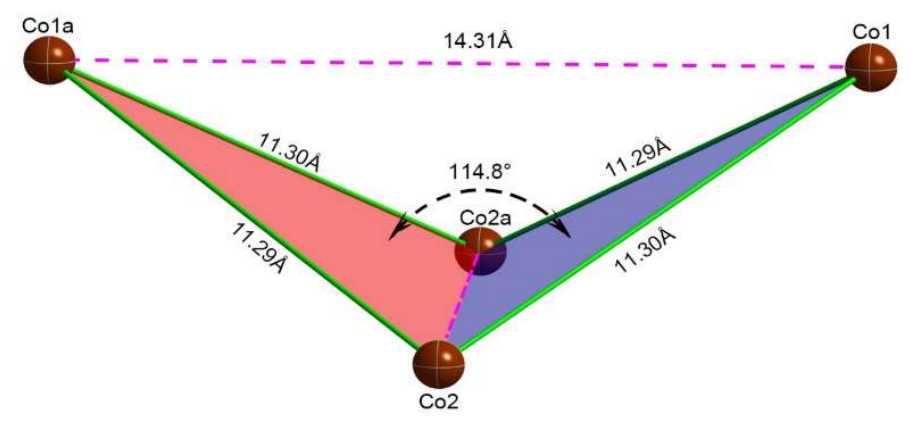

Figure S18. (a) ORTEP drawing of $\mathbf{3}$ with $40 \%$ thermal ellipsoids. All hydrogen atoms, solvents and counter ions were omitted for clarity (C: gray, N: blue, O: red, Co: orange red). Pentagonal bipyramidal coordination spheres are highlighted in green. (b) Simplified structural model of $\mathbf{3}$.

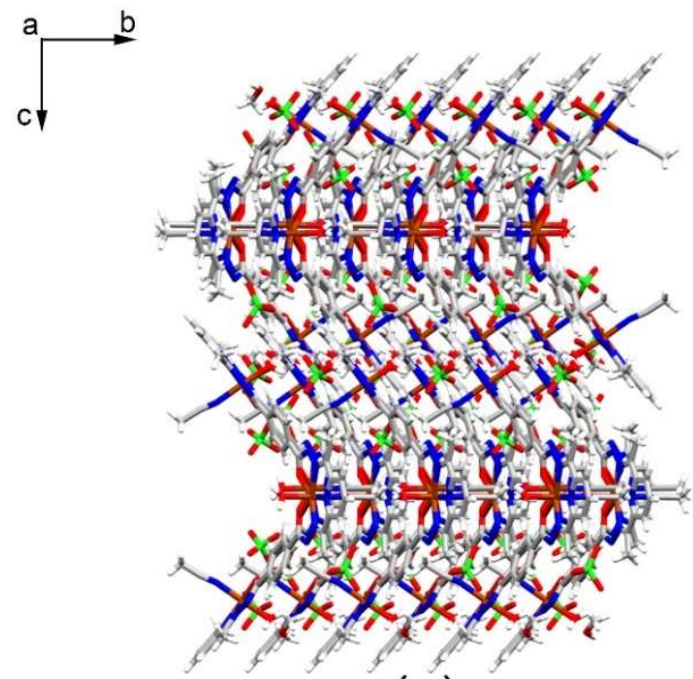

(a)

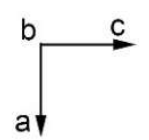

$c$

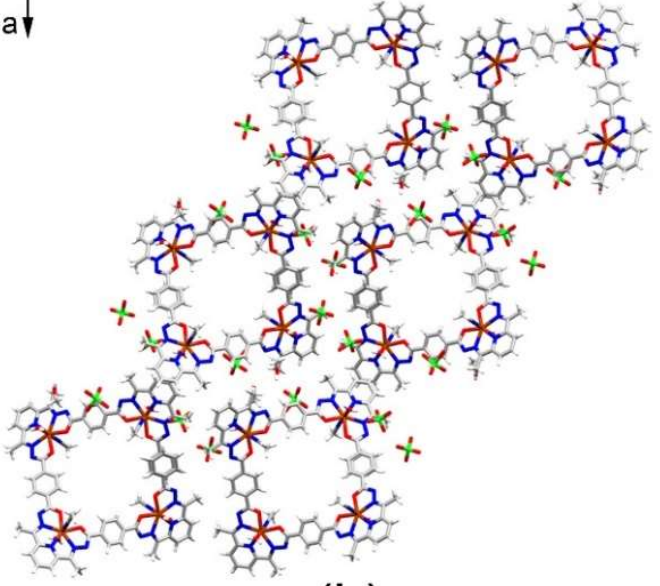

(b)

Figure S19. Packing diagram of 3 along the a-axis (a) and b-axis (b), respectively. 
Table S5. Selected Bond Distances $(\AA)$ and Angles (deg) for 3.

\begin{tabular}{|c|c|c|c|}
\hline \multicolumn{4}{|c|}{ Bond lengths } \\
\hline Bond & Length $(\AA)$ & Bond & Length $(\AA)$ \\
\hline Co1-O1 & $2.174(7)$ & $\mathrm{Co} 2-\mathrm{O} 5$ & $2.156(6)$ \\
\hline $\mathrm{Co} 1-\mathrm{O} 2$ & $2.184(6)$ & $\mathrm{Co} 2-\mathrm{O} 4$ & $2.194(6)$ \\
\hline Co1-O3 & $2.149(9)$ & $\mathrm{Co} 2-\mathrm{O} 6$ & $2.114(7)$ \\
\hline Co1-N2 & $2.194(9)$ & Co2-N10 & $2.183(7)$ \\
\hline Co1-N4 & $2.172(7)$ & $\mathrm{Co} 2-\mathrm{N} 8$ & $2.176(7)$ \\
\hline Co1-N6 & $2.116(12)$ & $\mathrm{Co} 2-\mathrm{N} 12$ & $2.106(9)$ \\
\hline Co1-N3 & $2.147(4)$ & Co2-N9 & $2.131(4)$ \\
\hline \multicolumn{4}{|c|}{ Bond angle } \\
\hline Bond angle & Degree $\left(^{\circ}\right)$ & Bond angle & Degree $\left(^{\circ}\right)$ \\
\hline O1-Co1-O2 & $75.3(2)$ & N8-Co2-O4 & $71.5(2)$ \\
\hline O1-Co1-N2 & $71.5(3)$ & N9-Co2-N10 & $70.8(3)$ \\
\hline N4-Co1-O2 & $72.1(3)$ & N9-Co2-N8 & $71.0(2)$ \\
\hline N3-Co1-N2 & $70.9(3)$ & $\mathrm{O} 5-\mathrm{Co} 2-\mathrm{O} 4$ & $76.0(2)$ \\
\hline N3-Co1-N4 & $70.5(2)$ & O5-Co2-N10 & $70.8(3)$ \\
\hline N6-Co1-O3 & $177.9(4)$ & N12-Co2-O6 & $177.6(3)$ \\
\hline
\end{tabular}


(a)

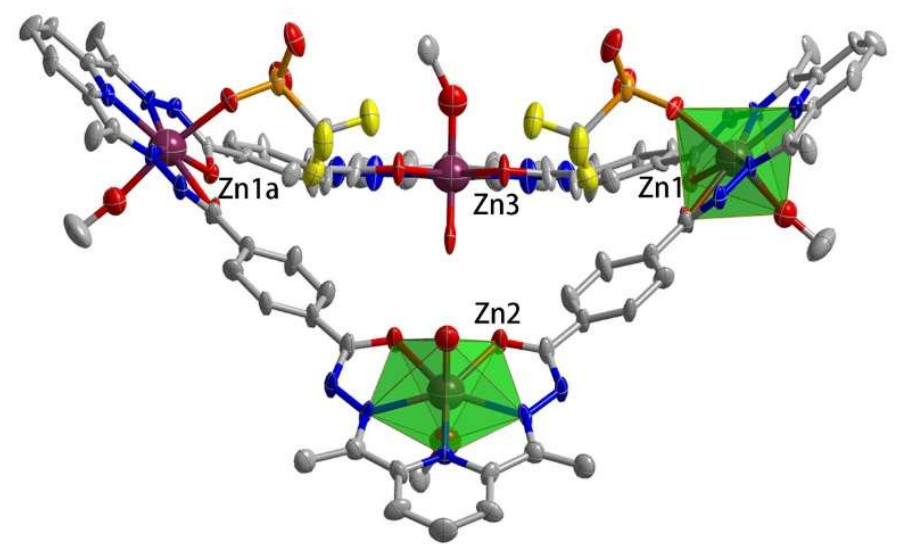

(b)

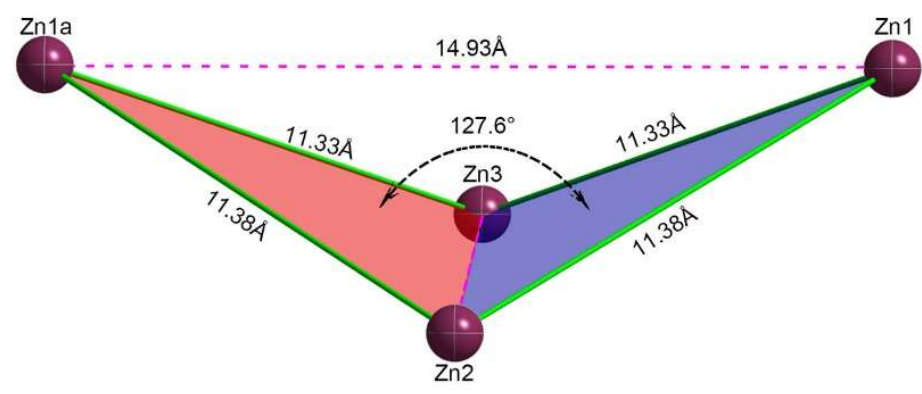

Figure S20. (a) ORTEP drawing of 4 with 30\% thermal ellipsoids. All hydrogen atoms and solvents were omitted for clarity (C: gray, N: blue, O: red, Zn: plum). Pentagonal bipyramidal coordination spheres are highlighted in green. (b) Simplified structural model of 4.

$\stackrel{\mathrm{b}}{\longrightarrow}$

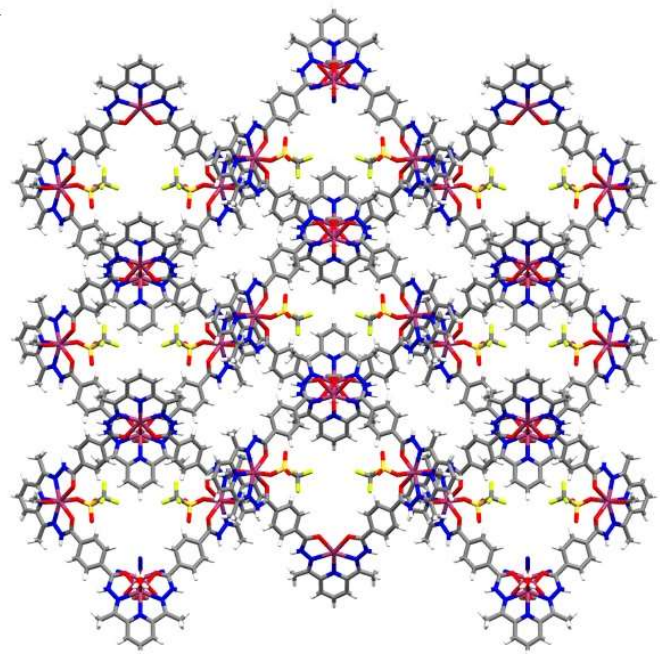

(a)

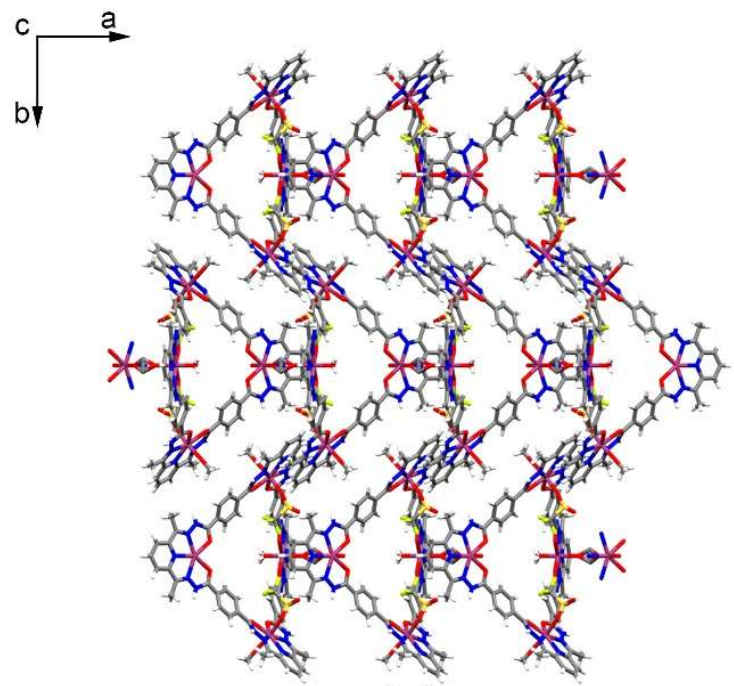

(b)

Figure S21. Packing diagram of $\mathbf{4}$ along the a-axis (a) and c-axis (b), respectively. 
Table S6. Selected Bond Distances ( $\AA$ ) and Angles (deg) for 4.

\begin{tabular}{|c|c|c|c|c|c|}
\hline \multicolumn{6}{|c|}{ Bond lengths } \\
\hline Bond & Length $(\AA)$ & Bond & Length $(\AA)$ & Bond & Length $(\AA)$ \\
\hline $\mathrm{Zn} 1-\mathrm{O} 2$ & $2.211(6)$ & $\mathrm{Zn} 2-\mathrm{O}^{\# 3}$ & $2.212(7)$ & $\mathrm{Zn} 3-\mathrm{N9} 9^{\# 1}$ & $2.208(10)$ \\
\hline Zn1-O1 & $2.243(6)$ & $\mathrm{Zn} 2-\mathrm{O} 7$ & $2.212(7)$ & $\mathrm{Zn} 3-\mathrm{O}^{\# 2}$ & $2.225(7)$ \\
\hline $\mathrm{Zn} 1-\mathrm{O} 4$ & $2.109(6)$ & $\mathrm{Zn} 2-\mathrm{N}^{\# 3}$ & $2.190(8)$ & $\mathrm{Zn} 3-\mathrm{O}^{\# 1}$ & $2.225(7)$ \\
\hline $\mathrm{Zn} 1-\mathrm{N} 2$ & $2.203(8)$ & Zn2-N7 & $2.190(8)$ & $\mathrm{Zn3}-\mathrm{N} 10^{\# 1}$ & $2.224(7)$ \\
\hline Zn1-N4 & $2.203(7)$ & $\mathrm{Zn} 2-\mathrm{O} 10$ & $2.051(17)$ & 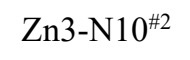 & $2.224(7)$ \\
\hline $\mathrm{Zn} 1-\mathrm{N} 3$ & $2.179(4)$ & $\mathrm{Zn} 2-\mathrm{N} 8$ & $2.226(14)$ & $\mathrm{Zn3}-\mathrm{O} 12$ & $2.181(16)$ \\
\hline $\mathrm{Zn} 1-\mathrm{O} 3$ & $2.069(10)$ & Zn2-O9 & $1.826(13)$ & $\mathrm{Zn3-O11}$ & $2.117(9)$ \\
\hline \multicolumn{6}{|c|}{ Bond angle } \\
\hline Bond angle & Degree $\left(^{\circ}\right)$ & Bond angle & Degree $\left(^{\circ}\right)$ & Bond angle & Degree $\left(^{\circ}\right)$ \\
\hline $\mathrm{N} 3-\mathrm{Zn} 1-\mathrm{N} 2$ & $69.6(2)$ & $\mathrm{O} 7-\mathrm{Zn} 2-\mathrm{N} 7$ & $72.1(3)$ & $\begin{array}{c}\text { N9 }{ }^{\# 1}-Z n 3- \\
N 10^{\# 1}\end{array}$ & $70.27(19)$ \\
\hline $\mathrm{N} 3-\mathrm{Zn} 1-\mathrm{N} 4$ & $70.1(2)$ & $\mathrm{O} 7-\mathrm{Zn} 2-\mathrm{O}^{\# 3}$ & $77.6(3)$ & $\begin{array}{c}\text { N9 }{ }^{\# 1}-\mathrm{Zn} 3- \\
\mathrm{N} 10^{\# 2}\end{array}$ & $70.27(19)$ \\
\hline $\mathrm{N} 2-\mathrm{Zn} 1-\mathrm{O} 1$ & $70.0(3)$ & N7-Zn2-N8 & $69.1(2)$ & $\begin{array}{c}\mathrm{O} 8^{\# 1}-\mathrm{Zn} 3- \\
\mathrm{O} 8^{\# 2}\end{array}$ & $77.3(4)$ \\
\hline $\mathrm{O} 3-\mathrm{Zn} 1-\mathrm{O} 4$ & $172.8(3)$ & $\mathrm{N} 8-\mathrm{Zn} 2-\mathrm{N} 7^{\# 3}$ & $69.1(2)$ & $\begin{array}{c}\mathrm{N} 10^{\# 1}-\mathrm{Zn} 3- \\
\mathrm{O} 8^{\# 1}\end{array}$ & $71.1(3)$ \\
\hline $\mathrm{N} 4-\mathrm{Zn} 1-\mathrm{O} 2$ & $70.9(2)$ & $\begin{array}{c}\mathrm{N}^{\# 3}-\mathrm{Zn} 2- \\
\mathrm{O}^{\# 3}\end{array}$ & $72.1(3)$ & $\begin{array}{c}\mathrm{N} 10^{\# 2}-\mathrm{Zn} 3- \\
\mathrm{O}^{\# 2}\end{array}$ & $71.1(3)$ \\
\hline $\mathrm{O} 2-\mathrm{Zn} 1-\mathrm{O} 1$ & $79.5(2)$ & O9-Zn-O10 & $170.6(5)$ & $\begin{array}{c}\text { O11-Zn3- } \\
\text { O12 }\end{array}$ & $177.7(5)$ \\
\hline
\end{tabular}

Symmetry transformations used to generate equivalent atoms:

\#1: $-1+\mathrm{X},+\mathrm{Y},-1+\mathrm{Z}$; \#2: -1+X, 3/2-Y, -1+Z; \#3: +X, 3/2-Y, +Z.

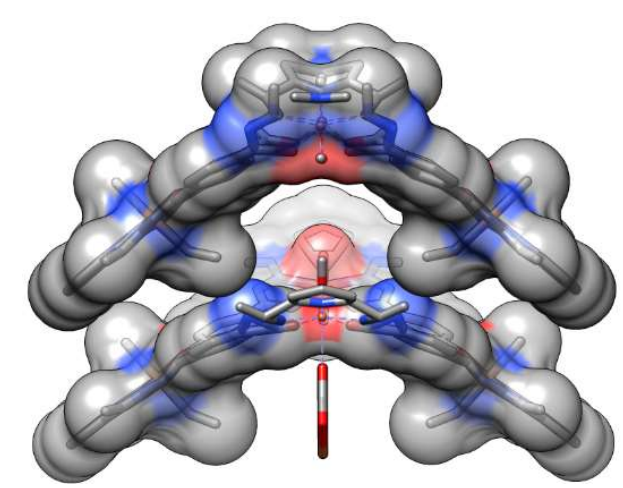

Figure S22. The preliminary analysis crystal structure of $\mathbf{5 .}$ 
(a)

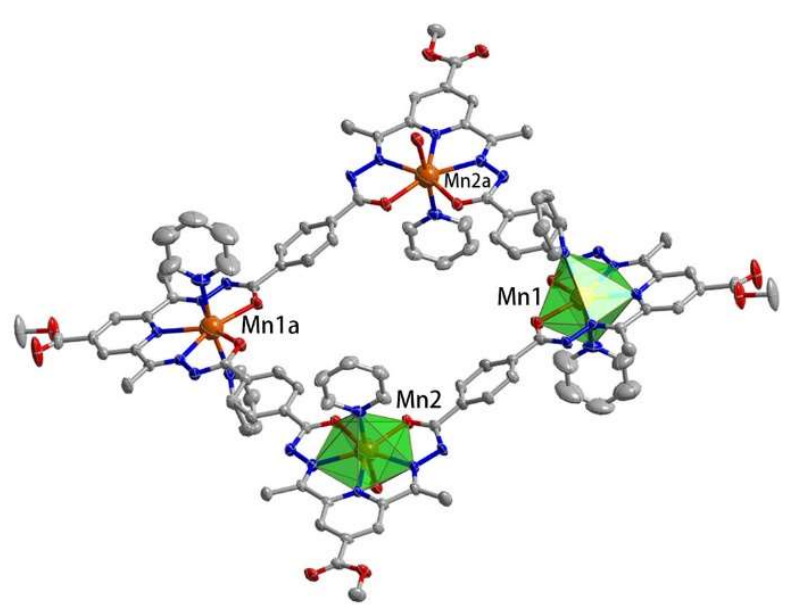

(b)

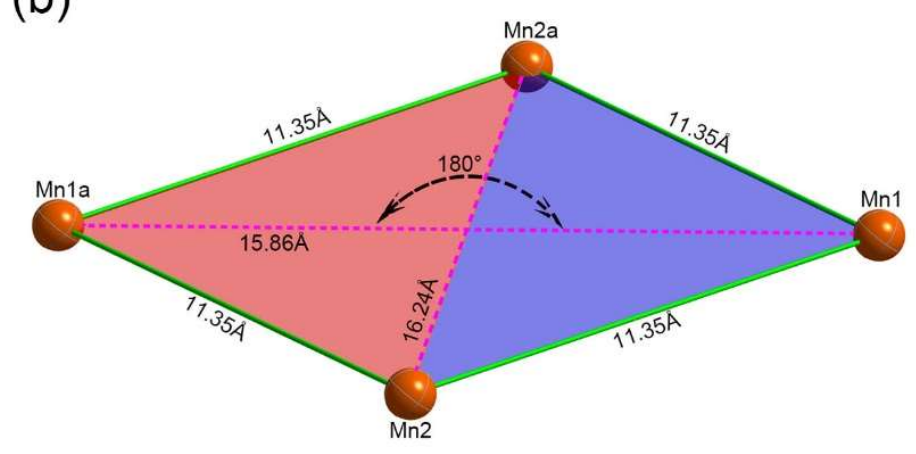

Figure S23. (a) ORTEP drawing of 6' with $60 \%$ thermal ellipsoids. All hydrogen atoms and solvents were omitted for clarity (C: gray, N: blue, O: red, Mn: orange). Pentagonal bipyramidal coordination spheres are highlighted in green. (b) Simplified structural model of 6'.

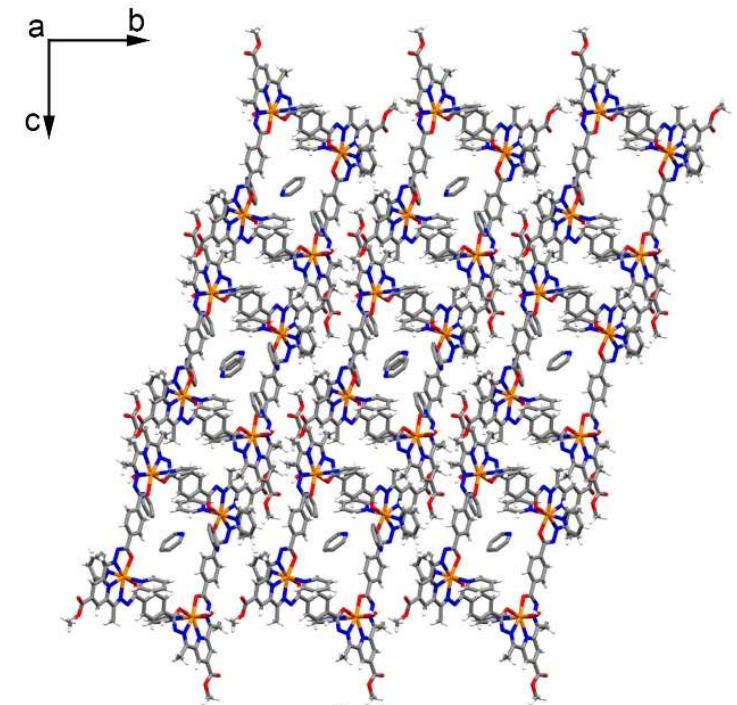

(a)<smiles>[CH]CBr</smiles>

\section{$\stackrel{a}{a}$}

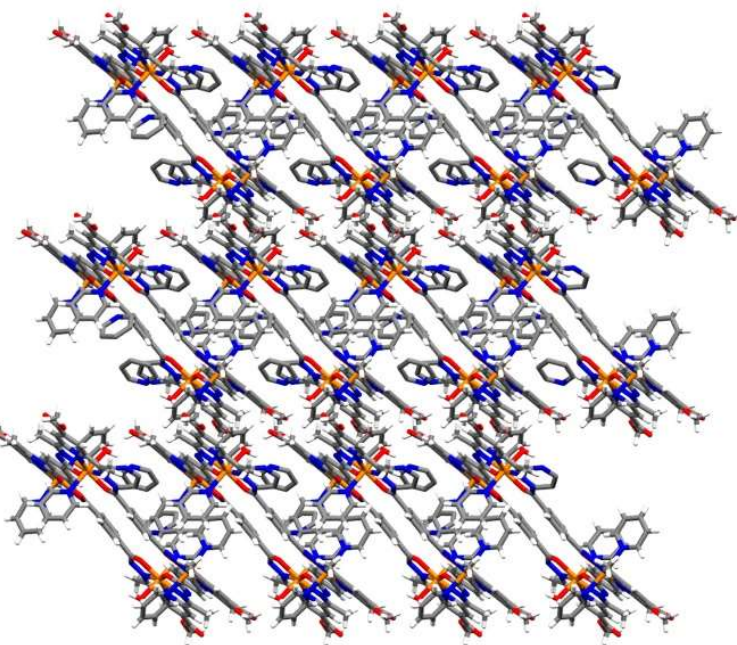

(b)

Figure S24. Packing diagram of 6' along the a-axis (a) and c-axis (b), respectively. 
Table S7. Selected Bond Distances $(\AA)$ and Angles (deg) for 6'.

\begin{tabular}{|c|c|c|c|}
\hline \multicolumn{4}{|c|}{ Bond lengths } \\
\hline Bond & Length $(\AA)$ & Bond & Length $(\AA)$ \\
\hline $\mathrm{Mn} 1-\mathrm{O} 2$ & $2.175(3)$ & $\mathrm{Mn} 2-\mathrm{O} 7$ & $2.200(3)$ \\
\hline Mn1-O1 & $2.229(3)$ & Mn2-O6 & $2.239(3)$ \\
\hline Mn1-O3 & $2.255(3)$ & Mn2-N9 & $2.345(3)$ \\
\hline Mn1-N4 & $2.317(3)$ & Mn2-N10 & $2.313(3)$ \\
\hline Mn1-N3 & $2.333(3)$ & Mn2-N12 & $2.311(4)$ \\
\hline Mn1-N2 & $2.290(3)$ & $\mathrm{Mn} 2-\mathrm{N} 8$ & $2.315(3)$ \\
\hline Mn1-N6 & $2.303(4)$ & $\mathrm{Mn} 2$-N13 & $2.278(3)$ \\
\hline \multicolumn{4}{|c|}{ Bond angle } \\
\hline Bond angle & Degree $\left({ }^{\circ}\right)$ & Bond angle & Degree $\left(^{\circ}\right)$ \\
\hline O1-Mn1-N2 & $69.19(11)$ & O7-Mn2-N10 & $69.41(11)$ \\
\hline O2-Mn1-O1 & $86.33(10)$ & O7-Mn2-O6 & $88.05(11)$ \\
\hline N4-Mn1-N3 & $67.14(11)$ & N8-Mn2-N9 & $67.16(11)$ \\
\hline N2-Mn1-N3 & $67.98(11)$ & O6-Mn2-N8 & $68.67(11)$ \\
\hline O2-Mn1-N4 & $69.37(11)$ & N10-Mn2-N9 & $66.81(12)$ \\
\hline O3-Mn1-N6 & $177.01(12)$ & N13-Mn2-N12 & $172.23(13)$ \\
\hline
\end{tabular}

Table S8. Results of Continuous Shape Measures calculation using SHAPE with the closest geometries for each seven-coordinate metal centers in asymmetry unit are highlighted in red.
S H A P E v 2.1
Continuous Shape Measures calculation

(c) 2013 Electronic Structure Group, Universitat de Barcelona

$\begin{array}{lll}\text { HP-7 } & 1 \mathrm{D}_{7 \mathrm{~h}} & \text { Heptagon } \\ \text { HPY-7 } & 2 \mathrm{C}_{6 \mathrm{v}} & \text { Hexagonal pyramid } \\ \text { PBPY-7 } & 3 \mathrm{D}_{5 \mathrm{~h}} & \text { Pentagonal bipyramid } \\ \text { COC-7 } & 4 \mathrm{C}_{3 \mathrm{v}} & \text { Capped octahedron } \\ \text { CTPR-7 } & 5 \mathrm{C}_{2 \mathrm{v}} & \text { Capped trigonal prism } \\ \text { JPBPY-7 } & 6 \mathrm{D}_{5 \mathrm{~h}} & \text { Johnson pentagonal bipyramid J13 } \\ \text { JETPY-7 } & 7 \mathrm{C}_{3 \mathrm{v}} & \text { Johnson elongated triangular pyramid J7 }\end{array}$




\begin{tabular}{cccccccc}
\hline complex & HP-7 & HPY-7 & PBPY-7 & COC-7 & CTPR-7 & JPBPY-7 & JETPY-7 \\
\hline $\mathbf{1}$ & 32.084, & 20.324, & $\mathbf{0 . 7 8 6}$, & 6.628, & 5.097, & 4.144, & $20.694,20.012$ \\
& 31.897 & 19.924 & $\mathbf{1 . 0 8 5}$ & 6.183 & 4.446 & 4.453 & \\
$\mathbf{2}$ & 33.269, & 21.447, & $\mathbf{0 . 5 3 2}$, & 7.250, & 5.703, & 4.322, & $21.605,22.227$ \\
& 33.687 & 23.038 & $\mathbf{0 . 6 3 1}$ & 6.992 & 5.249 & 4.593 & \\
$\mathbf{3}$ & 34.519, & 25.267, & $\mathbf{0 . 1 0 8}$, & 7.355, & 5.582, & 3.301, & $23.989,23.902$ \\
& 34.067 & 24.568 & $\mathbf{0 . 1 0 7}$ & 7.735 & 5.960 & 3.268 & \\
$\mathbf{4}$ & 33.419, & 22.846, & $\mathbf{0 . 1 4 0 ,}$ & 6.818, & 5.343, & 2.913, & $22.804,24.096$, \\
& 34.361, & 24.336, & $\mathbf{0 . 3 9 7}$, & 7.601, & 5.994, & 2.956, & 22.997 \\
& 31.952 & 20.099 & $\mathbf{1 . 0 7 8}$ & 7.825 & 6.381 & 1.612 & \\
\multirow{2}{*}{$\boldsymbol{*}$} & 32.776, & 21.030, & $\mathbf{0 . 4 7 8}$, & 6.570, & 5.031, & 4.565, & $21.139,22.384$ \\
& 33.281 & 22.270 & $\mathbf{0 . 8 2 2}$ & 7.454 & 5.988 & 4.217 & \\
\hline
\end{tabular}

Table S9. summary parameters of metallacycles 1-4 and 6.

\begin{tabular}{ccccc}
\hline metallacycles & angle $(\alpha)^{\circ}$ & $\begin{array}{c}\text { distance between } \\
\text { the adjacent metal } \\
\text { centers }(\AA)\end{array}$ & $\begin{array}{c}\text { distance between } \\
\text { the opposite metal } \\
\text { centers }(\AA)\end{array}$ & angle (M-M-M) ${ }^{\circ}$ \\
\hline $\mathbf{1}$ & $89.03,88.58$ & $11.38,11.48$ & $16.15,16.18$ & $89.87,90.13$ \\
$\mathbf{2}$ & $89.21,88.82$ & $15.72,15.73$ & 22.24 & 90 \\
$\mathbf{3}$ & $81.17,80.00$ & $11.29,11.30$ & $14.31,14.91$ & $78.60,82.54$ \\
$\mathbf{4}$ & $84.17,85.96$, & $11.33,11.38$ & $14.93,15.45$ & $82.00,82.36$, \\
& 88.02 & & & 85.82 \\
$\mathbf{6}$ & $87.04,89.65$ & 11.35 & $15.86,16.24$ & $88.66,91.34$ \\
\hline
\end{tabular}


8. Thermogravimetric analysis and UV-Vis spectra.

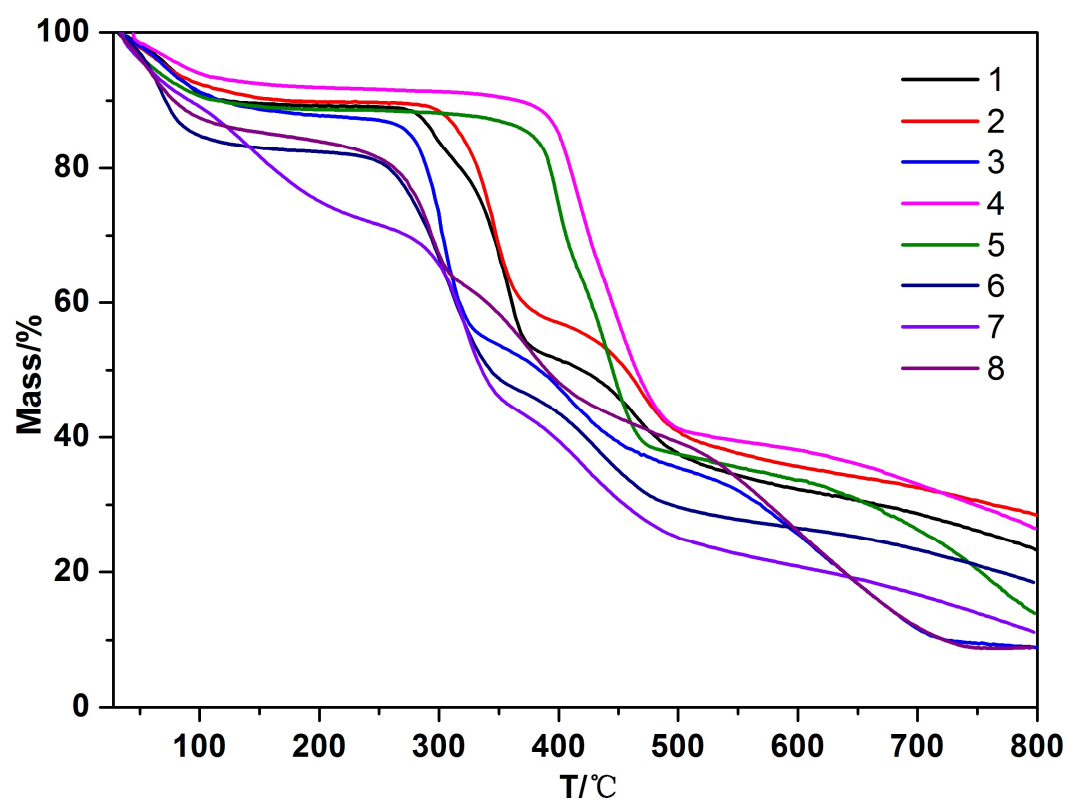

Figure S25. Thermogravimetric curves of 1-8.

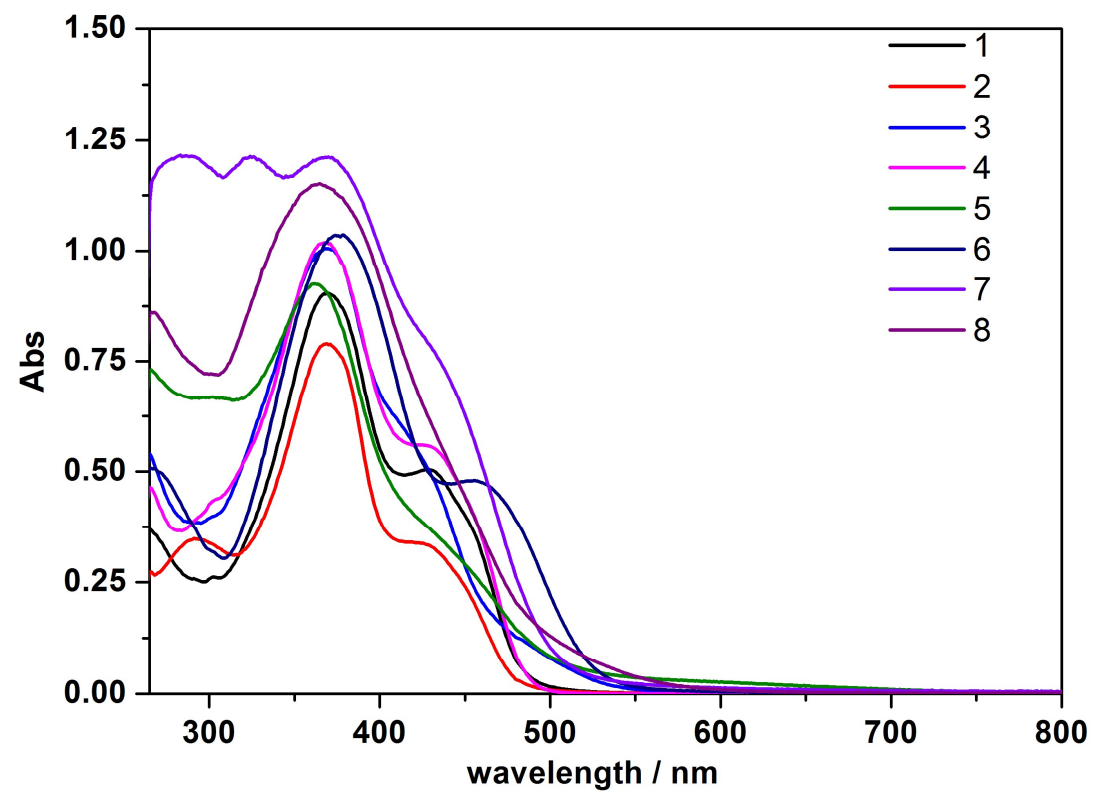

Figure S26. UV-Vis spectra of 1-8 $(6.67 \mu \mathrm{M})$ in DMF. 


\section{Characterization of aerogels.}
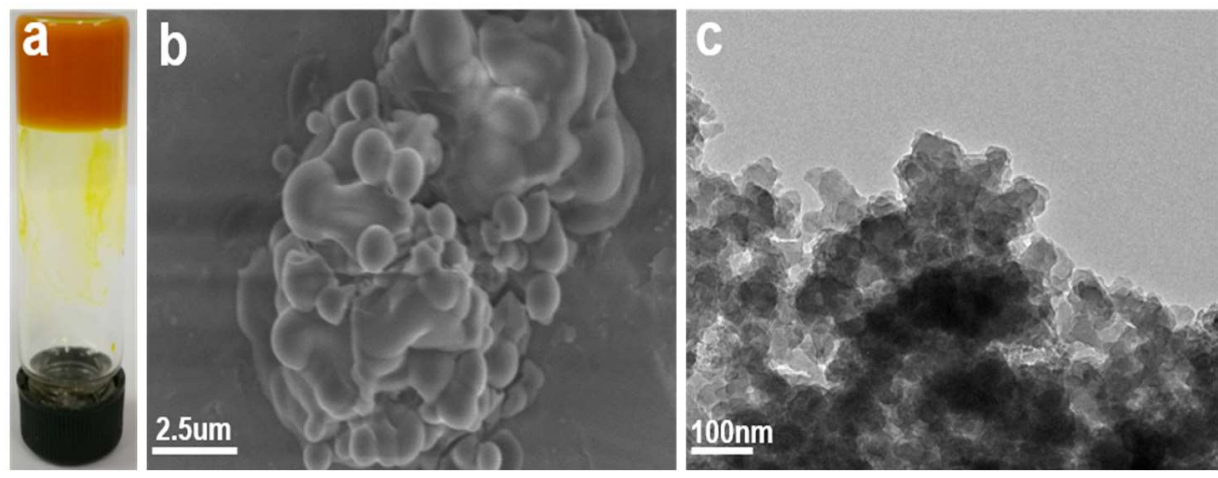

Figure S27. a) Photographic, b) SEM and c) TEM images of 7-Al.

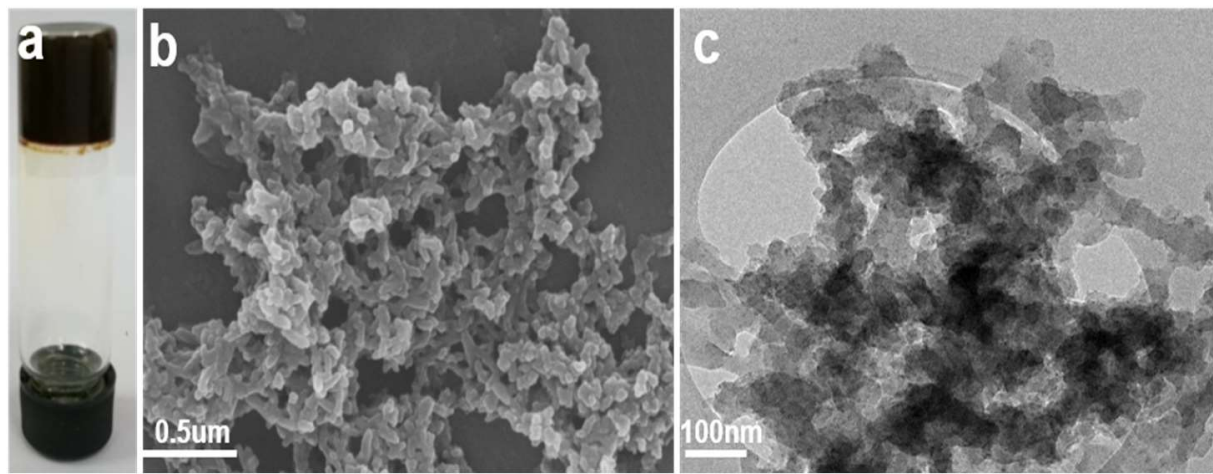

Figure S28. a) Photographic, b) SEM and c) TEM images of 7-Fe.
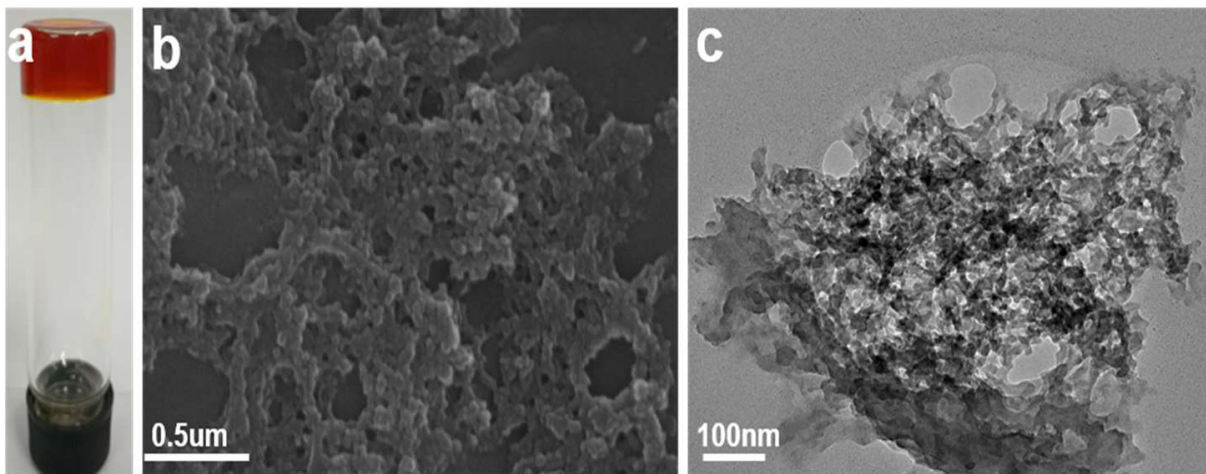

Figure S29. a) Photographic, b) SEM and c) TEM images of 8-Al. 


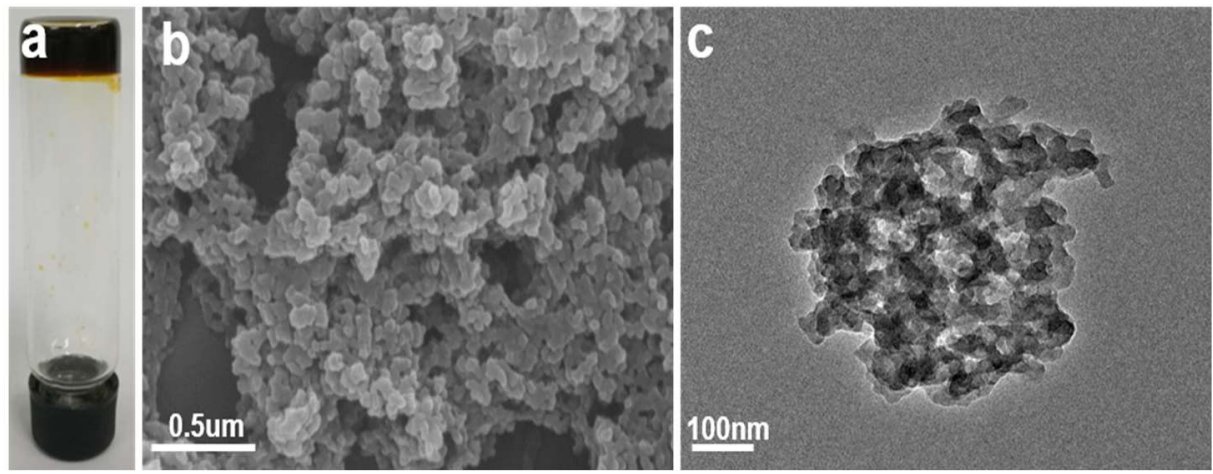

Figure S30. a) Photographic, b) SEM and c) TEM images of 8-Fe.

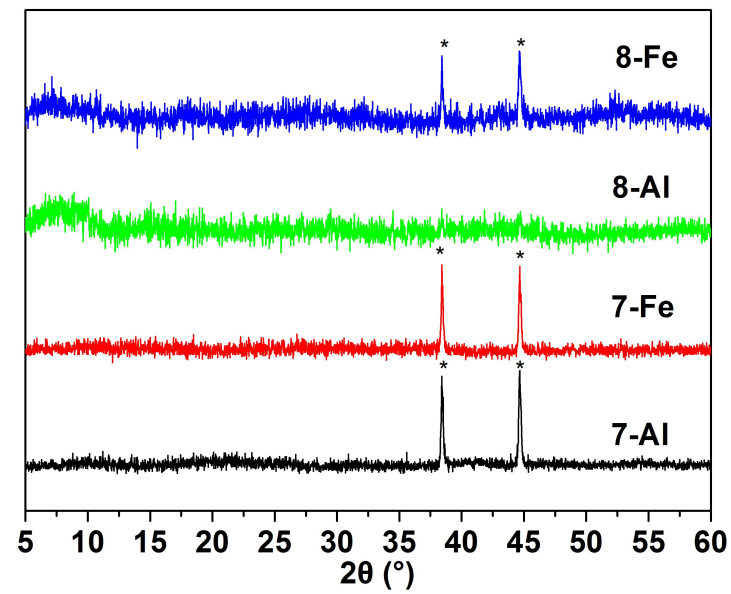

Figure S31. PXRD patterns of the corresponding aerogels, the peaks marked with * were attributed to silicon carrier.
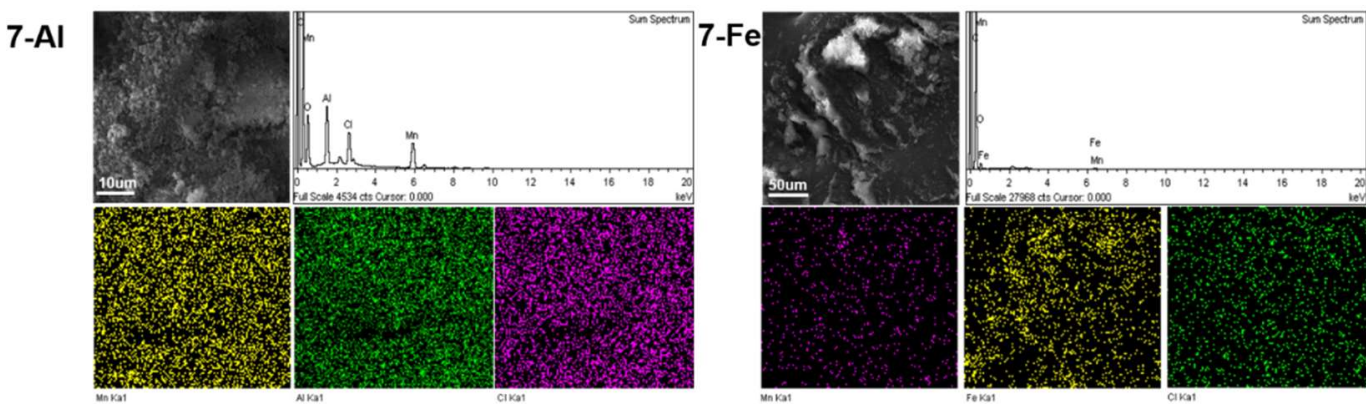

8-Al
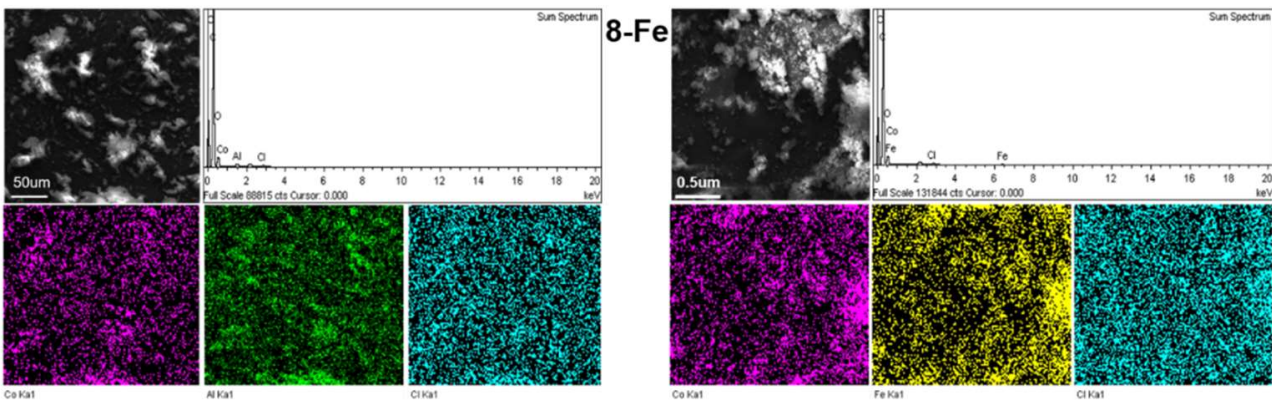

Figure S32. SEM energy dispersive X-ray spectra and elemental mapping of the corresponding aerogels. 
Table S10. Porosity properties of the aerogels.

\begin{tabular}{ccccc}
\hline Aerogel & $\mathrm{SA}_{\mathrm{BET}}{ }^{\mathrm{a})} / \mathrm{m}^{2} \mathrm{~g}^{-1}$ & $\mathrm{~V}_{\mathrm{t}} \mathrm{b} / \mathrm{cm}^{3} \mathrm{~g}^{-1}$ & $\mathrm{~V}_{\text {micro }} \mathrm{c} / \mathrm{cm}^{3} \mathrm{~g}^{-1}$ & $\mathrm{~V}_{\text {meso }}{ }^{\mathrm{d})} / \mathrm{cm}^{3} \mathrm{~g}^{-1}$ \\
\hline 7-Al & 61 & 0.32 & -- & 0.32 \\
7-Fe & 226 & 0.54 & 0.04 & 0.49 \\
8-Al & 207 & 0.96 & -- & 0.96 \\
8-Fe & 122 & 0.60 & -- & 0.60 \\
\hline
\end{tabular}

SABET is the BET specific surface area in the units of $\left.\mathrm{m}^{2} \mathrm{~g}^{-1} . \mathrm{b}\right) \mathrm{V}_{\mathrm{t}}$ is the total specific pore volume. c) $\mathrm{V}_{\text {micro }}$ is the specific micropore volume calculated using the t-plot method. d) $V_{\text {meso }}$ is the specific mesopore volume calculated using the BJH method.

\section{0. $\mathrm{CO}_{2}$ capture and conversion.}

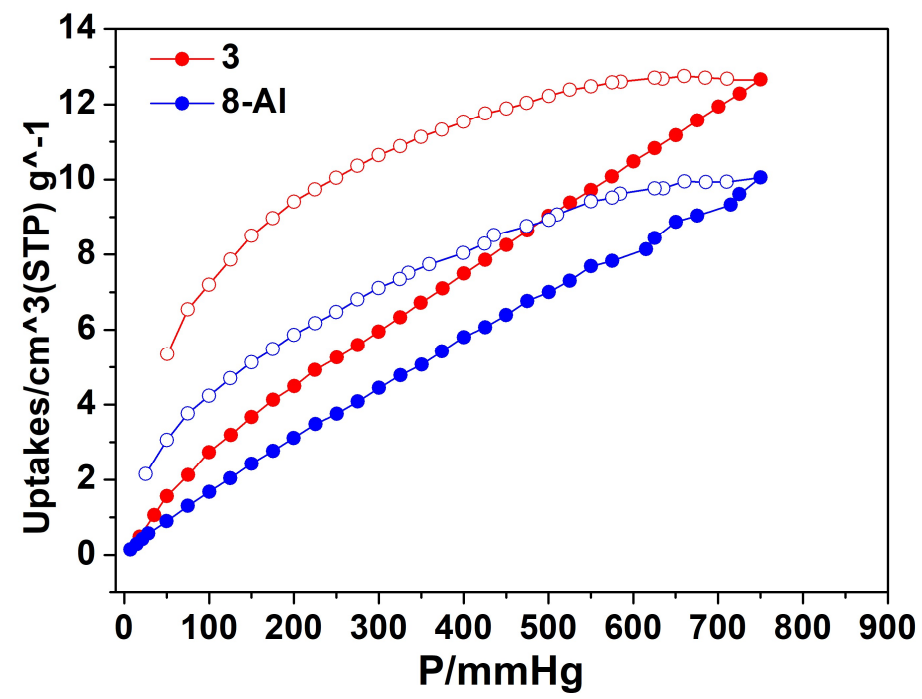

Figure S33. $\mathrm{CO}_{2}$ isotherm curves measured at $298 \mathrm{~K}$ of $\mathbf{3}$ and $\mathbf{8 - A l}$ (solid circles for sorption and open circles for desorption).

\section{General procedure for catalytic reactions}

The reaction was typically carried out in in a $10 \mathrm{~mL}$ Schlenk tube using the epoxide ( $25 \mathrm{mmol})$ with $\mathrm{CO}_{2}$ purged at $1 \mathrm{~atm}$ under solvent free environment at room temperature catalyzed by the selected catalyst (Table S11) and co-catalyst of tetra-n-tertbutylammonium bromide (TBAB, $0.58 \mathrm{~g}$ ) for 48 hours. Subsequently, a small amount of the reaction mixture was sampled, diluted with $\mathrm{CDCl}_{3}$ and filtered through a syringe filter with $0.22 \mu \mathrm{m}$ pore size to remove the solid catalyst. The solution was then analyzed by ${ }^{1} \mathrm{H}$ NMR spectroscopy to calculate the conversion of epoxides to cyclic carbonates. 
Table S11. Generation of various cyclic carbonates from corresponding epoxides catalyzed by PBP Co-based metallacycles or aerogel. ${ }^{\text {[a] }}$

\begin{tabular}{|c|c|c|c|c|}
\hline entry & catalysts & Epoxides & Products & Yield[\%] \\
\hline $1^{[\mathrm{b}]}$ & 3 & & & 74 \\
\hline $2^{[\mathrm{b}]}$ & 8-Al & & & 95 \\
\hline $3^{[\mathrm{d}]}$ & -- & & & 20 \\
\hline $4^{[c]}$ & 3 & & & 88 \\
\hline $5^{[\mathrm{c}]}$ & 8-Al & & & 91 \\
\hline $6^{[\mathrm{d}]}$ & -- & & & 30 \\
\hline $7^{[\mathrm{cc}]}$ & 3 & & & 58 \\
\hline $8^{[\mathrm{c}]}$ & 8-Al & & & 52 \\
\hline $9^{[\mathrm{c}]}$ & 1 & & & 45 \\
\hline $10^{[\mathrm{cc}]}$ & 4 & & & 43 \\
\hline $11^{[\mathrm{c}]}$ & 5 & & & 52 \\
\hline $12^{[\mathrm{d}]}$ & -- & & & 16 \\
\hline $13^{[\mathrm{c}]}$ & 3 & & $=0$ & 40 \\
\hline $14^{[\mathrm{c}]}$ & 8-Al & & $\tau_{0}^{0}=0$ & 37 \\
\hline $15^{[\mathrm{d}]}$ & -- & 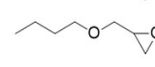 & $\tau_{0}^{0}>0$ & 11 \\
\hline $16^{[\mathrm{c}]}$ & 3 & 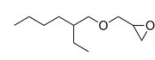 & $\tau_{0}^{0}=0$ & 10 \\
\hline $17^{[\mathrm{c}]}$ & 8-Al & $\checkmark 0$ & $r_{0}^{0}=0$ & 10 \\
\hline $18^{[\mathrm{d}]}$ & -- & $>0$ & $\tau_{0}^{0}=0$ & 6 \\
\hline
\end{tabular}

[a] Reaction conditions: epoxide $(25.0 \mathrm{mmol}), \mathrm{TBAB}(0.58 \mathrm{~g})$, under $1 \mathrm{~atm} \mathrm{CO}_{2}$ at room temperature for 48 hours; the yields were determined by ${ }^{1} \mathrm{H}$ NMR. [b] catalyst $(0.49 \%, 0.122 \mathrm{mmol}$ active site based on ICP-OES results). [c] catalyst $(0.12 \%, 0.03 \mathrm{mmol}$ active site based on ICP-OES results). [d] the same reaction conditions as in [a], but without the catalyst. 


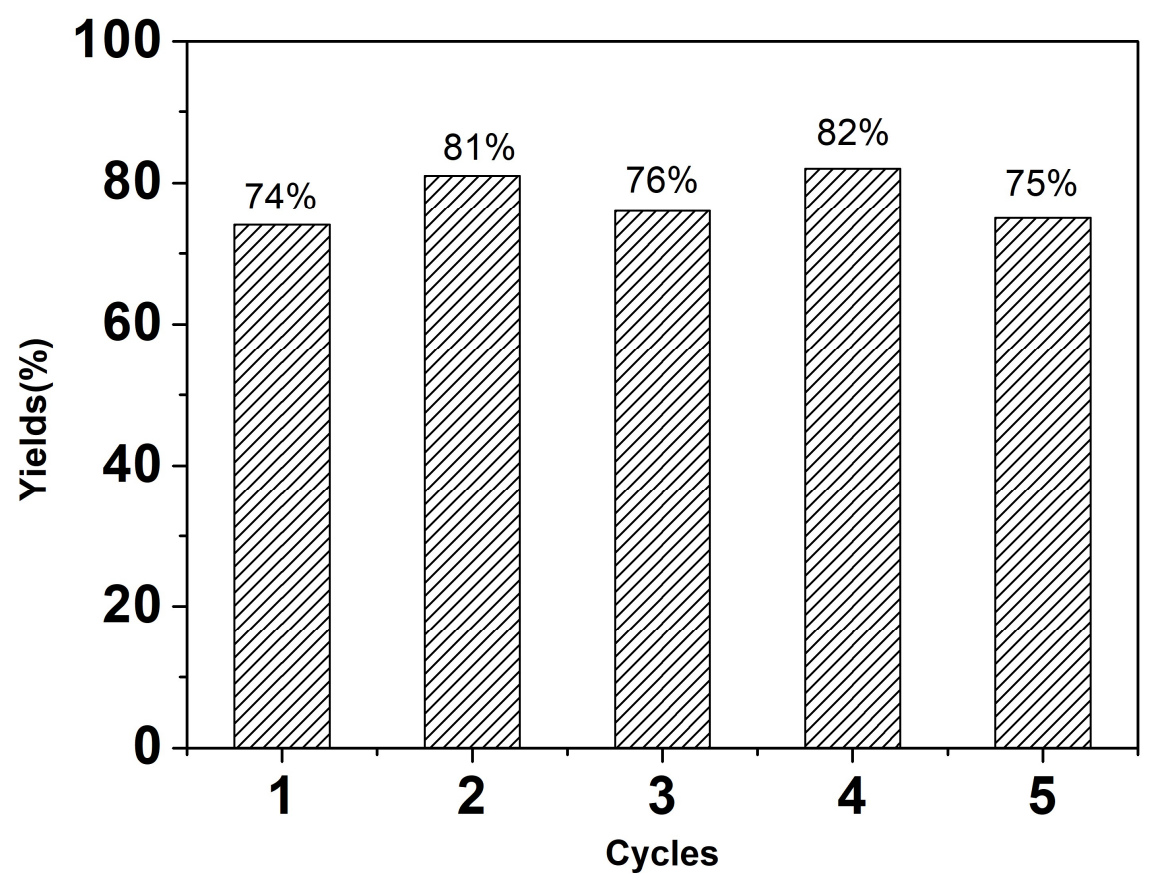

Figure S34. recyclability characterization of $\mathbf{3}$ in five cycles for catalytic $\mathrm{CO}_{2}$ cycloaddition with propylene oxide to produce propylene carbonate.

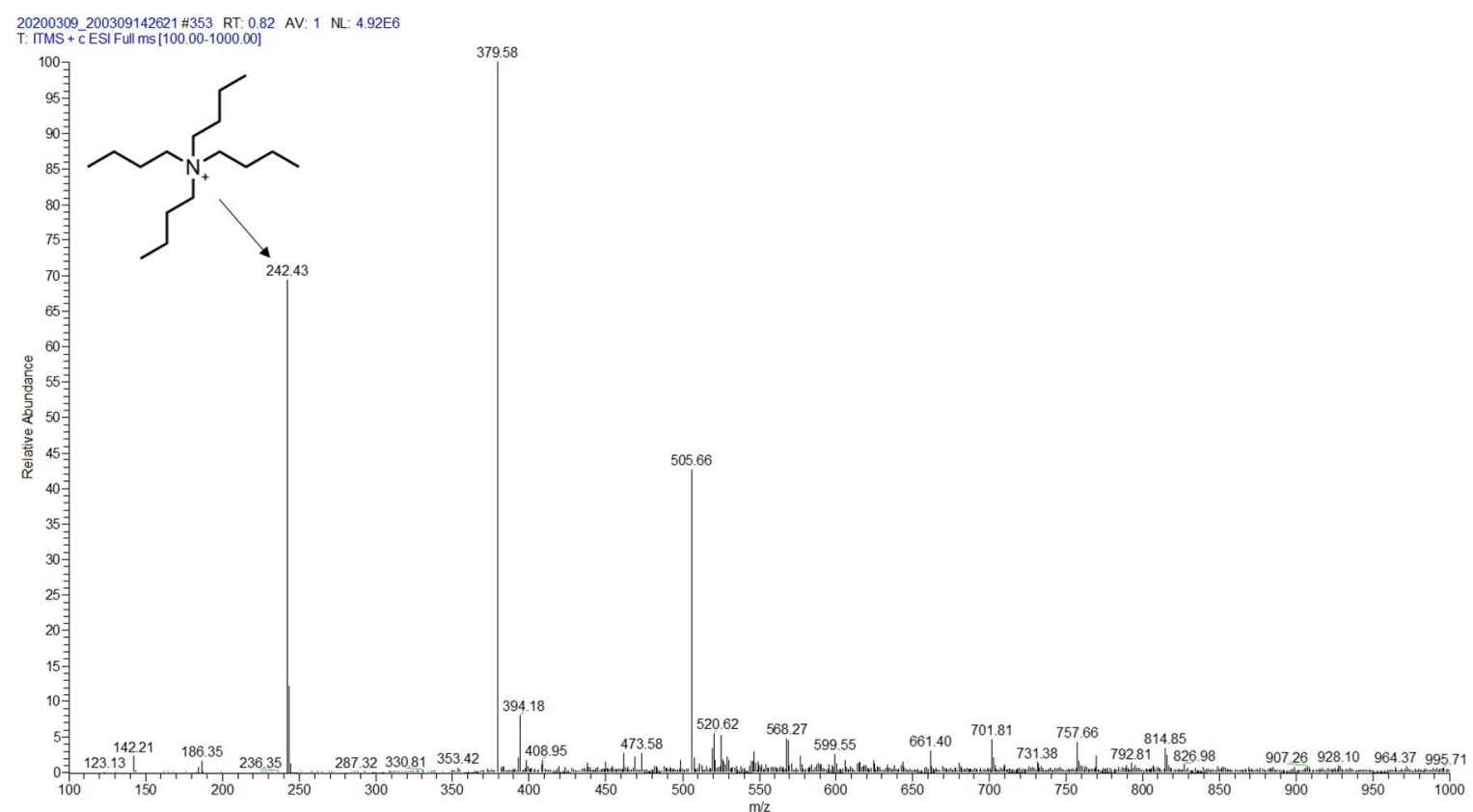

Figure S35. ESI-MS of 3 recollected after catalysis reactions matchs well with pristine 3 (Figure S5), indicating that the skeleton of $\mathbf{3}$ was retained after the catalysis reactions. 

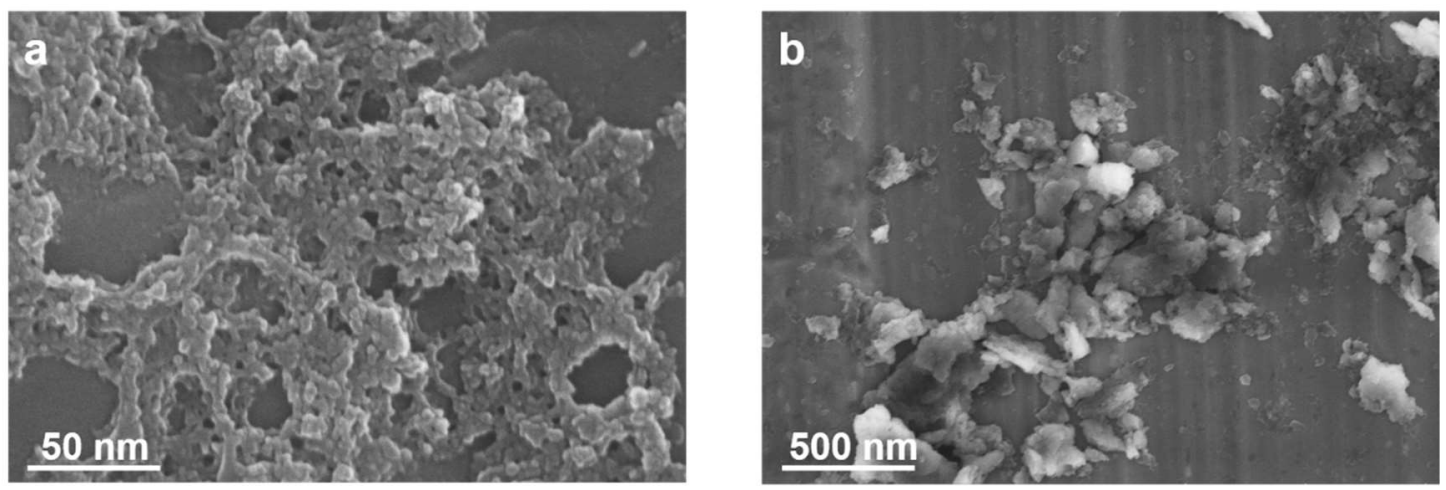

Figure S36. SEM images of 8-Al, a) before catalytic reactions, b) after catalytic reactions.

Table S12. Different epoxides coupled with $\mathrm{CO}_{2}$ catalyzed by salen-M catalysts under ambient conditions ( $1 \mathrm{~atm} \mathrm{CO}_{2}$ at room temperature).

\begin{tabular}{|c|c|c|c|c|}
\hline Entry & Epoxide & Catalyst & $\operatorname{TOF}\left(\mathrm{h}^{-1}\right)^{\mathrm{c}}$ & References \\
\hline $1^{\mathrm{a}}$ & & Co-CMP 100 & 3.5 & S6 \\
\hline $2^{\mathrm{a}}$ & 0 & Al-CMP 95.2 & 3.3 & S6 \\
\hline $3^{\mathrm{a}}$ & 0 & Cr-CMP & 3.2 & S7 \\
\hline $4^{b}$ & & Al(III)@cage & 3.7 & S8 \\
\hline \multirow[t]{3}{*}{$5^{\mathrm{a}}$} & & Salen-tBu- & 2.5 & S9 \\
\hline & & Cu(II)@MIL- & & \\
\hline & & $101(\mathrm{Cr})$ & & \\
\hline $6^{\mathrm{a}}$ & 0 & 3 & 3.2 & This work \\
\hline $7^{\mathrm{a}}$ & & 8-Al & 4.1 & This work \\
\hline $8^{a}$ & 0 & Cr-CMP & 3.1 & S7 \\
\hline $9^{b}$ & 0 & Al(III)@cage & 5.7 & 8 \\
\hline $10^{\mathrm{a}}$ & O & 8-Al & 15.8 & This work \\
\hline
\end{tabular}

[a] Reaction conditions: epoxide (25.0 mmol), TBAB (0.58 g), 48 hours.

[b] Reaction conditions: 1 mmol epoxide, 0.33 mol\% Al(III)@cage, 10 mol\% TBAB, 48 hours.

[c] Moles of products (CCs) produced per mole of active site $(\mathrm{M})$ per hour: $\mathrm{TOF}=$ moles of product/ (moles of catalyst $\mathrm{x}$ time). 


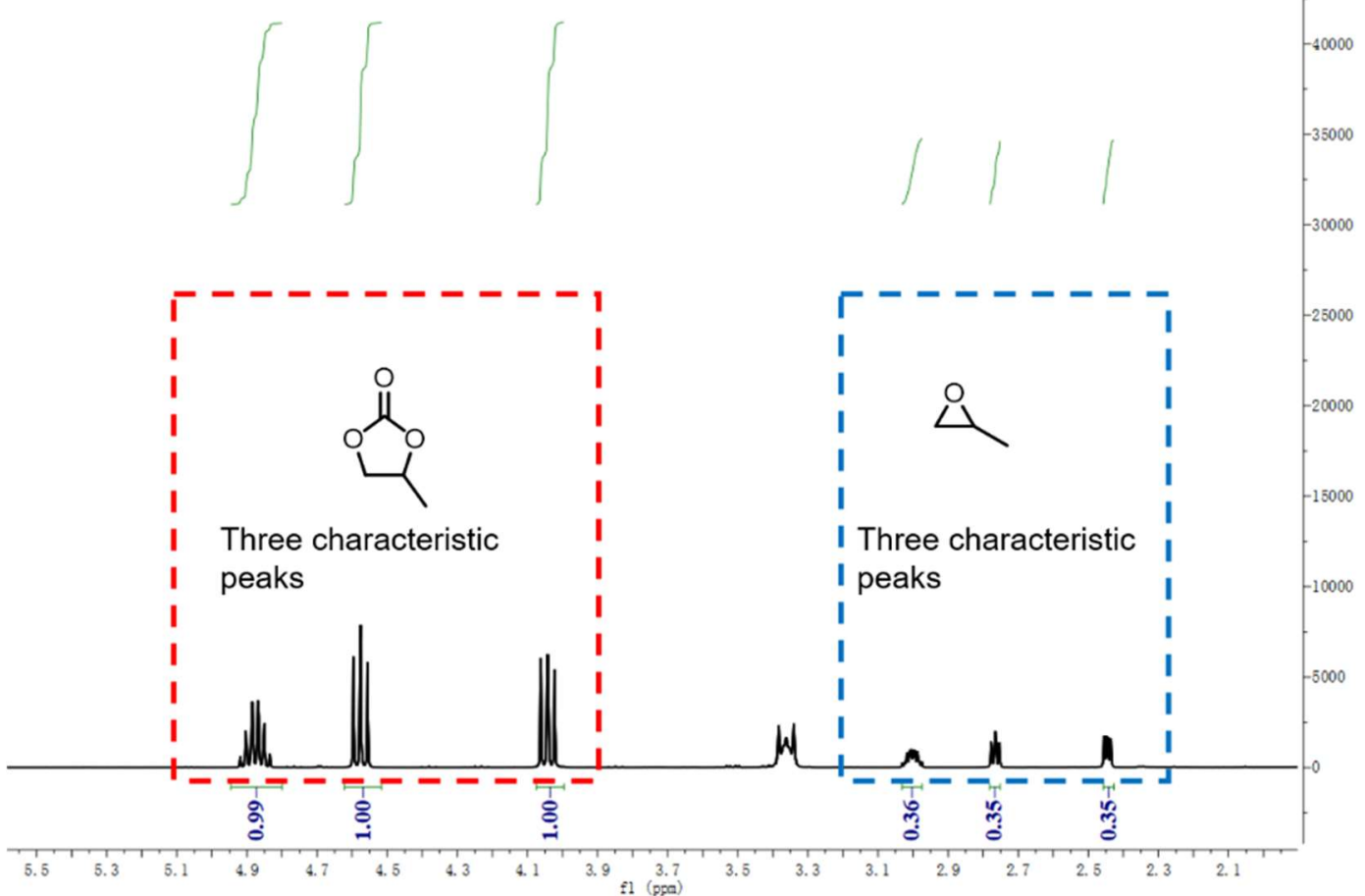

Figure S37. The ${ }^{1} \mathrm{H}-\mathrm{NMR}$ spectrum of propylene oxide and propylene carbonate by $\mathbf{3}$ in $\mathrm{CDCl}_{3}$.

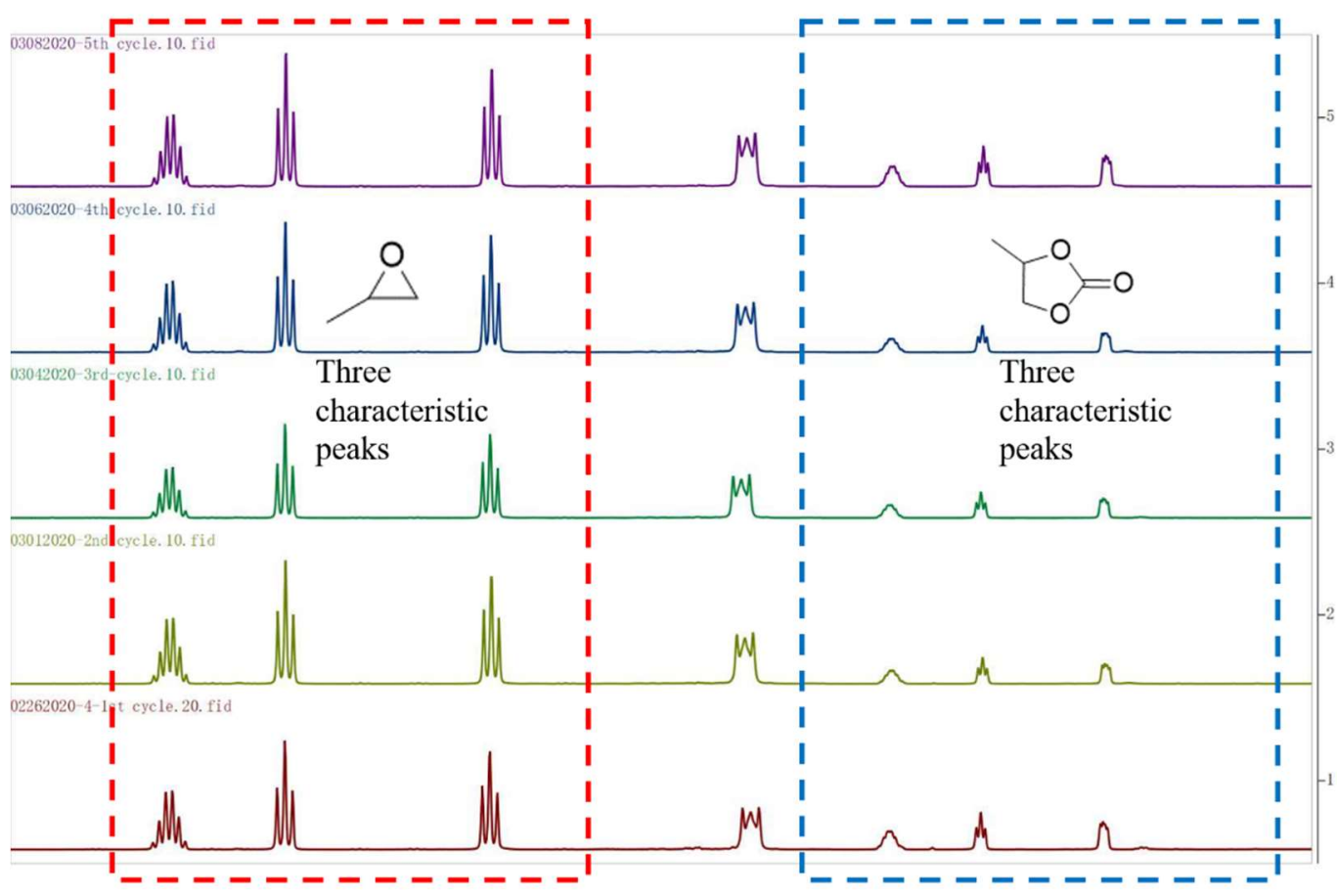

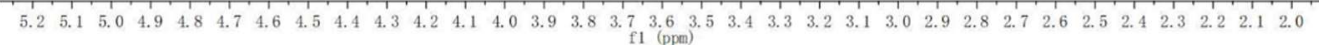

Figure S38. The multiple catalytic runs ${ }^{1} \mathrm{H}-\mathrm{NMR}$ spectrum of propylene oxide and propylene carbonate by 3 in $\mathrm{CDCl}_{3}$. 
$20180930-4-48 h$

PROTON CDC13 \{E: Idata2!1mzrxss\} lazmxss 5

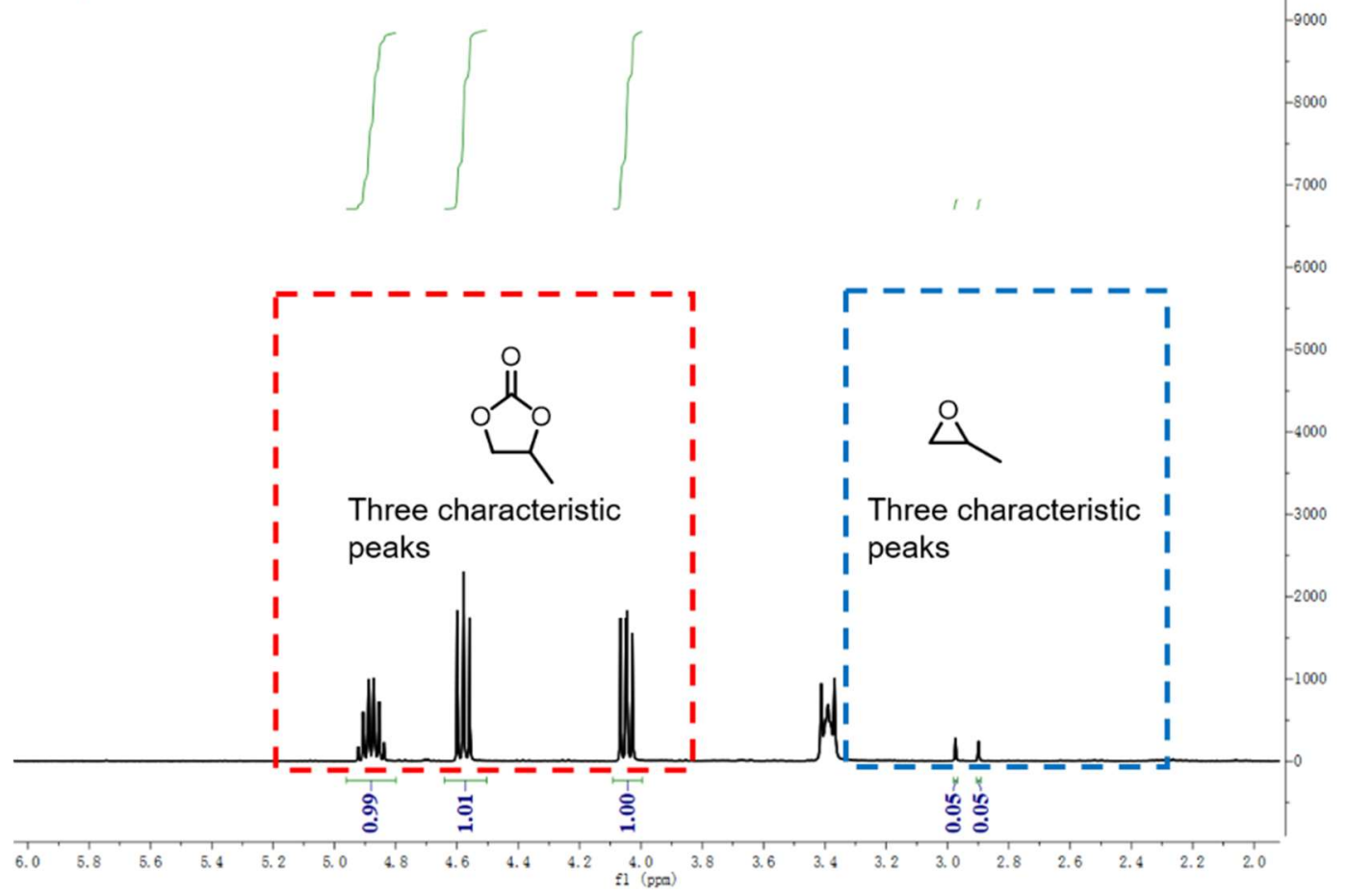

Figure S39. The 1 st cycle ${ }^{1} \mathrm{H}-\mathrm{NMR}$ spectrum of propylene oxide and propylene carbonate by $\mathbf{8}$-Al in $\mathrm{CDCl}_{3}$.

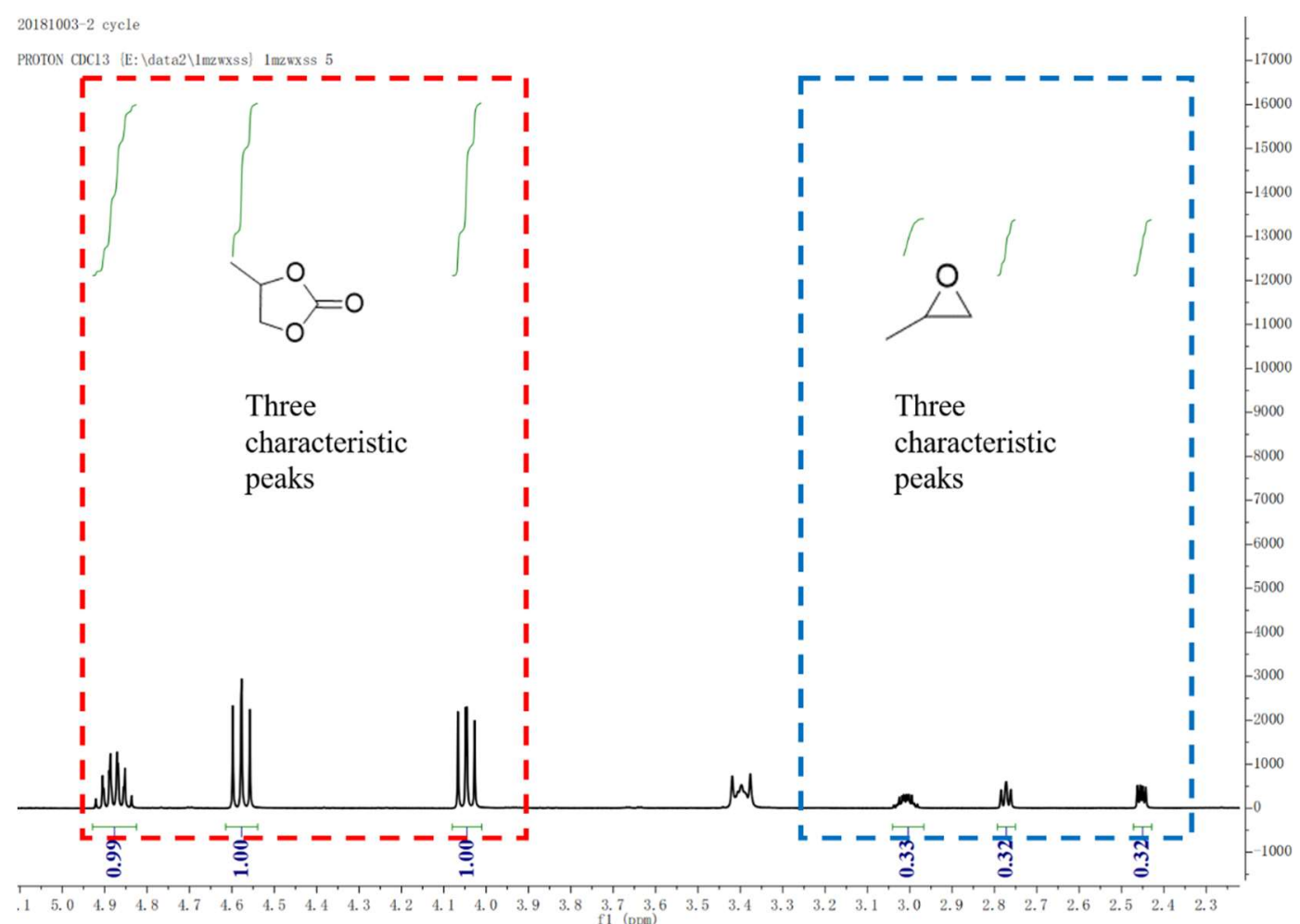

Figure S40. The 2 nd cycle ${ }^{1} \mathrm{H}-\mathrm{NMR}$ spectrum of propylene oxide and propylene carbonate by $\mathbf{8}$-Al in $\mathrm{CDCl}_{3}$. 


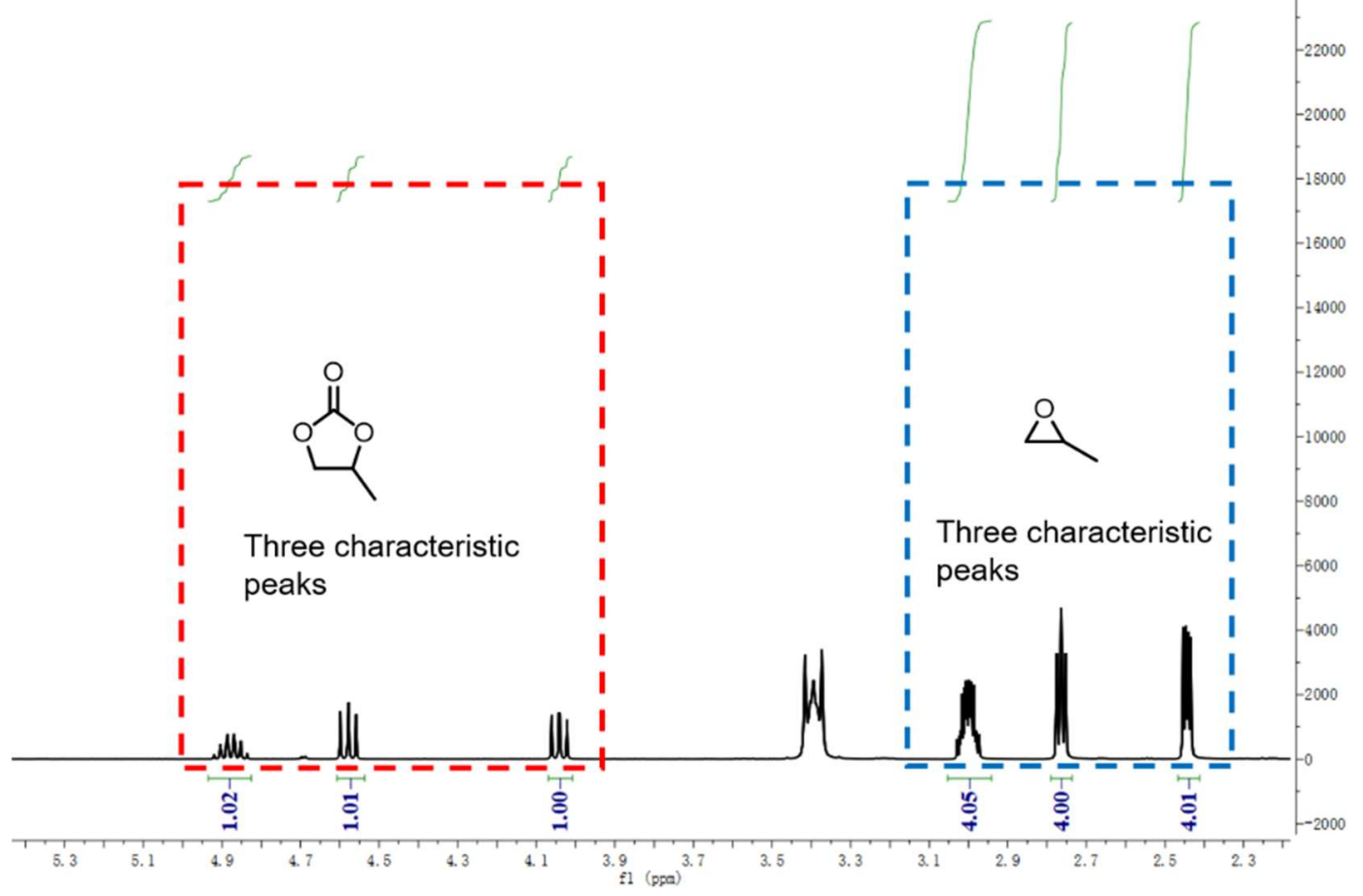

Figure S41. The ${ }^{1} \mathrm{H}-\mathrm{NMR}$ spectrum of propylene oxide and propylene carbonate by TBABr without catalyst in $\mathrm{CDCl}_{3}$.

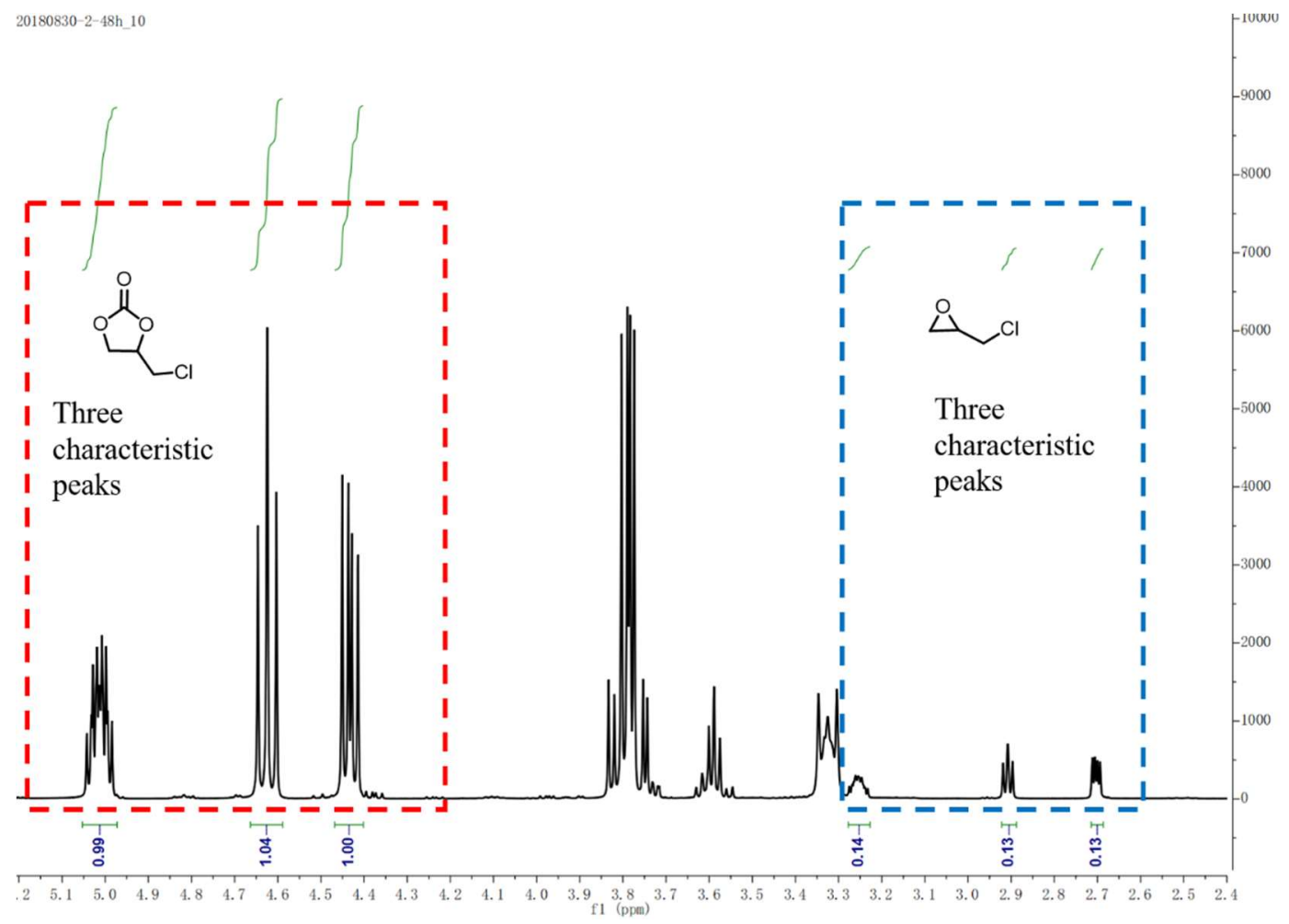

Figure S42. The ${ }^{1} \mathrm{H}-\mathrm{NMR}$ spectrum of 2-(chloromethyl) oxirane and 3-Chloropropene carbonate by 3 in $\mathrm{CDCl}_{3}$. 


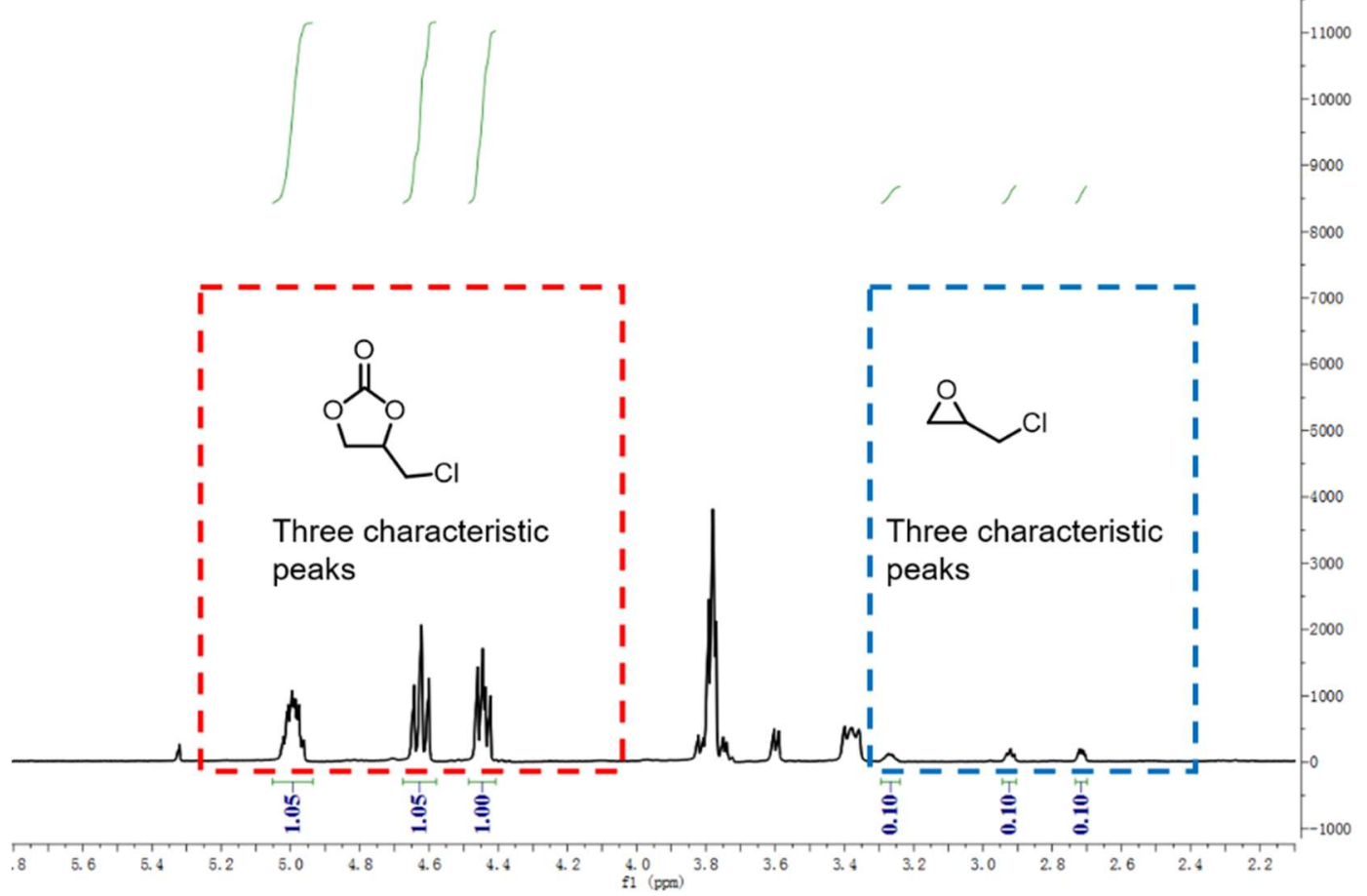

Figure S43. The ${ }^{1} \mathrm{H}-\mathrm{NMR}$ spectrum of 2-(chloromethyl) oxirane and 3-Chloropropene carbonate by 8$\mathrm{Al}$ in $\mathrm{CDCl}_{3}$.

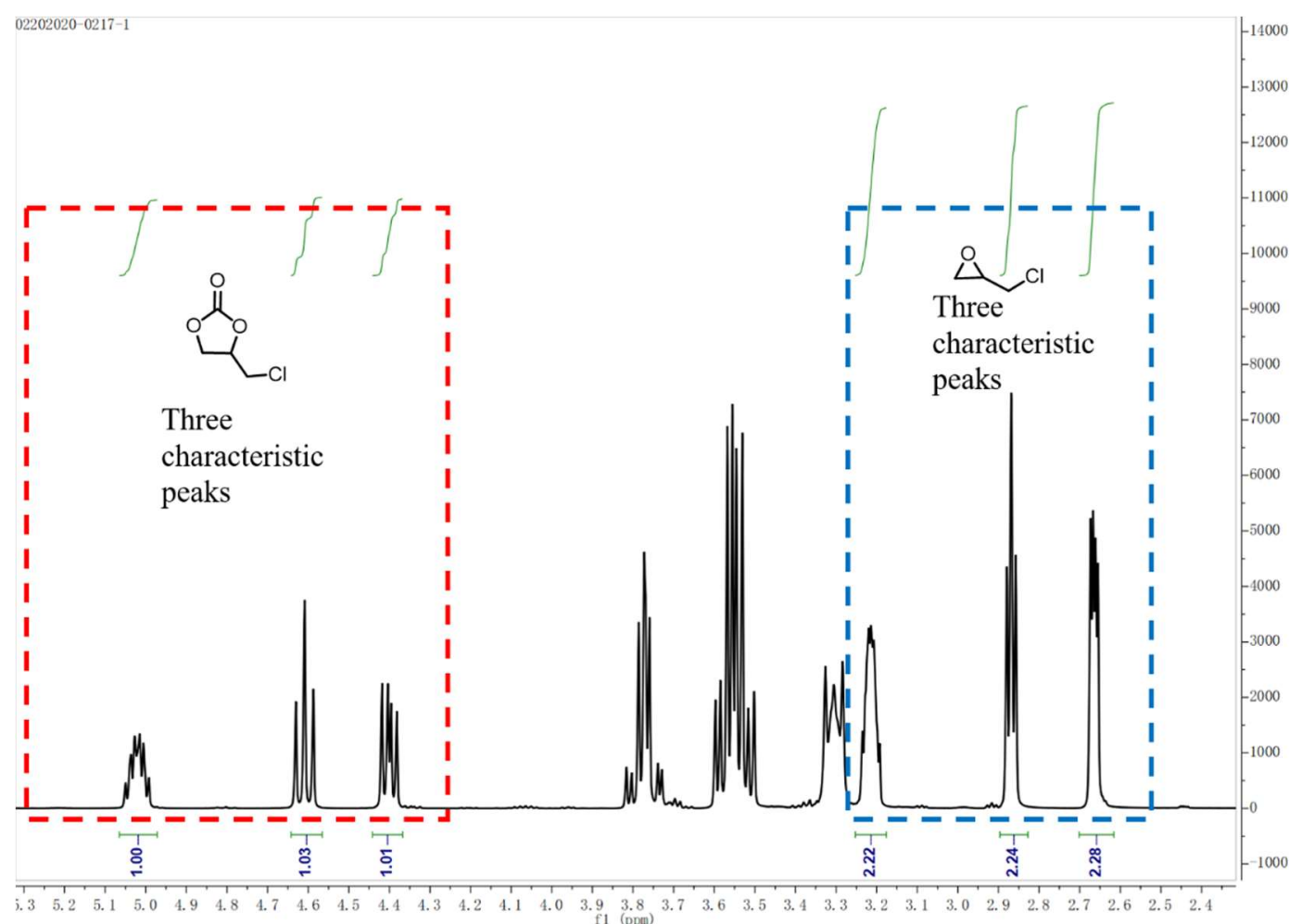

Figure S44. The ${ }^{1} \mathrm{H}-\mathrm{NMR}$ spectrum of 2-(chloromethyl) oxirane and 3-Chloropropene carbonate by TBABr without catalyst in $\mathrm{CDCl}_{3}$. 


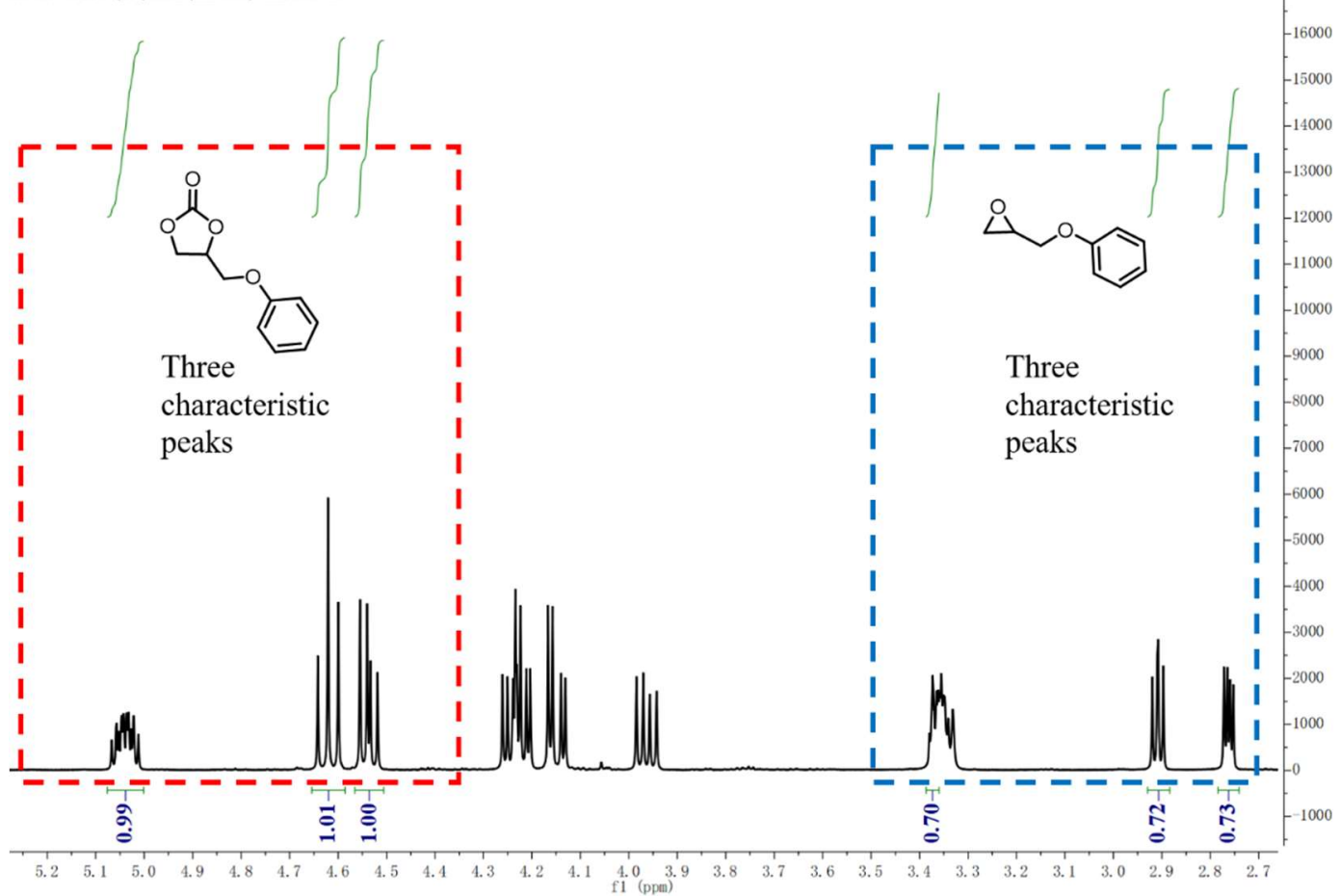

Figure S45. The ${ }^{1} \mathrm{H}-\mathrm{NMR}$ spectrum of benzyl phenyl glycidyl ether and 3-phenoxy-1,2-propylene carbonate by 3 in $\mathrm{CDCl}_{3}$.

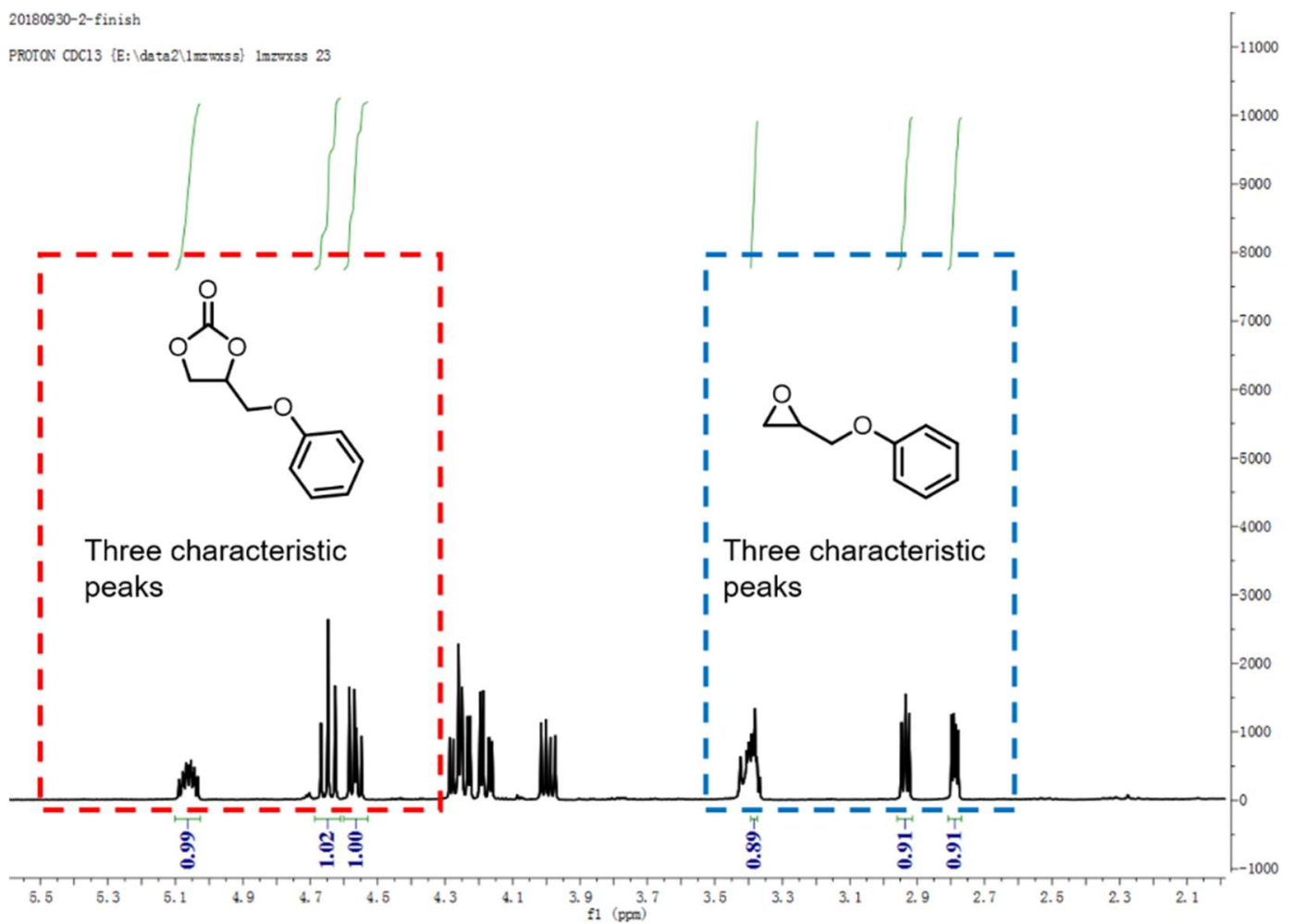

Figure S46. The ${ }^{1} \mathrm{H}-\mathrm{NMR}$ spectrum of benzyl phenyl glycidyl ether and 3-phenoxy-1,2-propylene carbonate by $\mathbf{8}-\mathbf{A l}$ in $\mathrm{CDCl}_{3}$. 


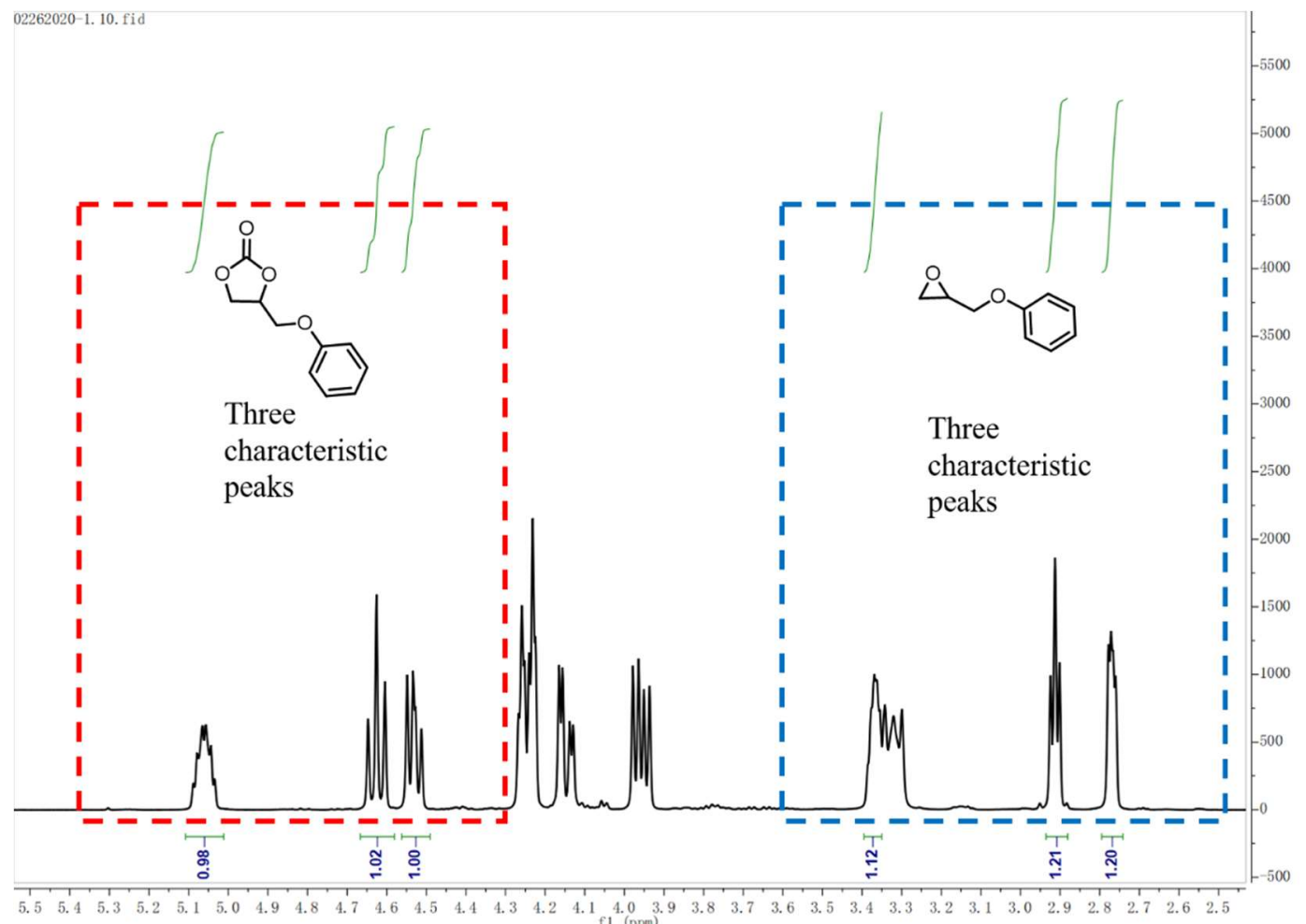

Figure S47. The ${ }^{1}$ H-NMR spectrum of benzyl phenyl glycidyl ether and 3-phenoxy-1,2-propylene carbonate by 1 in $\mathrm{CDCl}_{3}$.

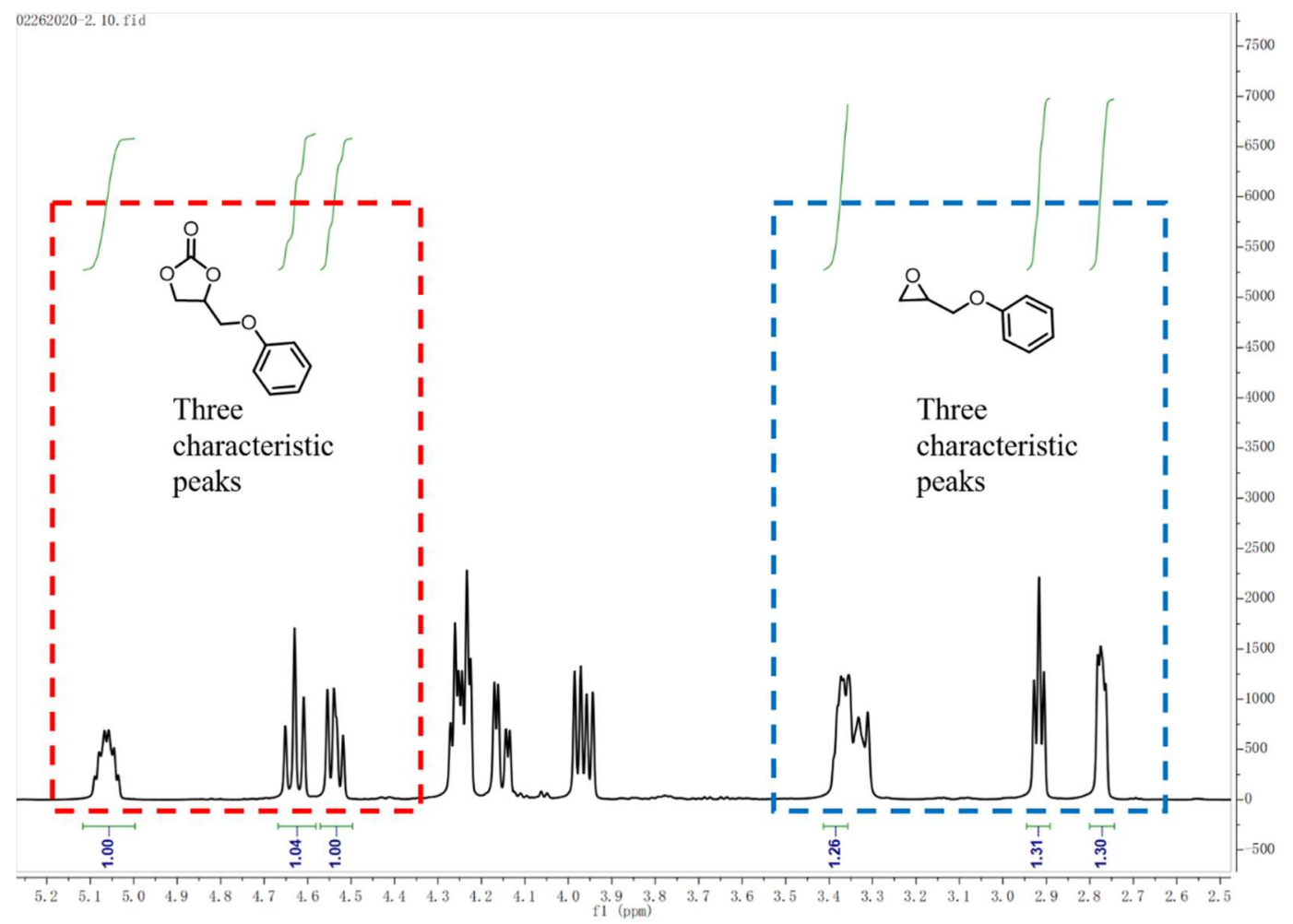

Figure S48. The ${ }^{1}$ H-NMR spectrum of benzyl phenyl glycidyl ether and 3-phenoxy-1,2-propylene carbonate by 4 in $\mathrm{CDCl}_{3}$. 


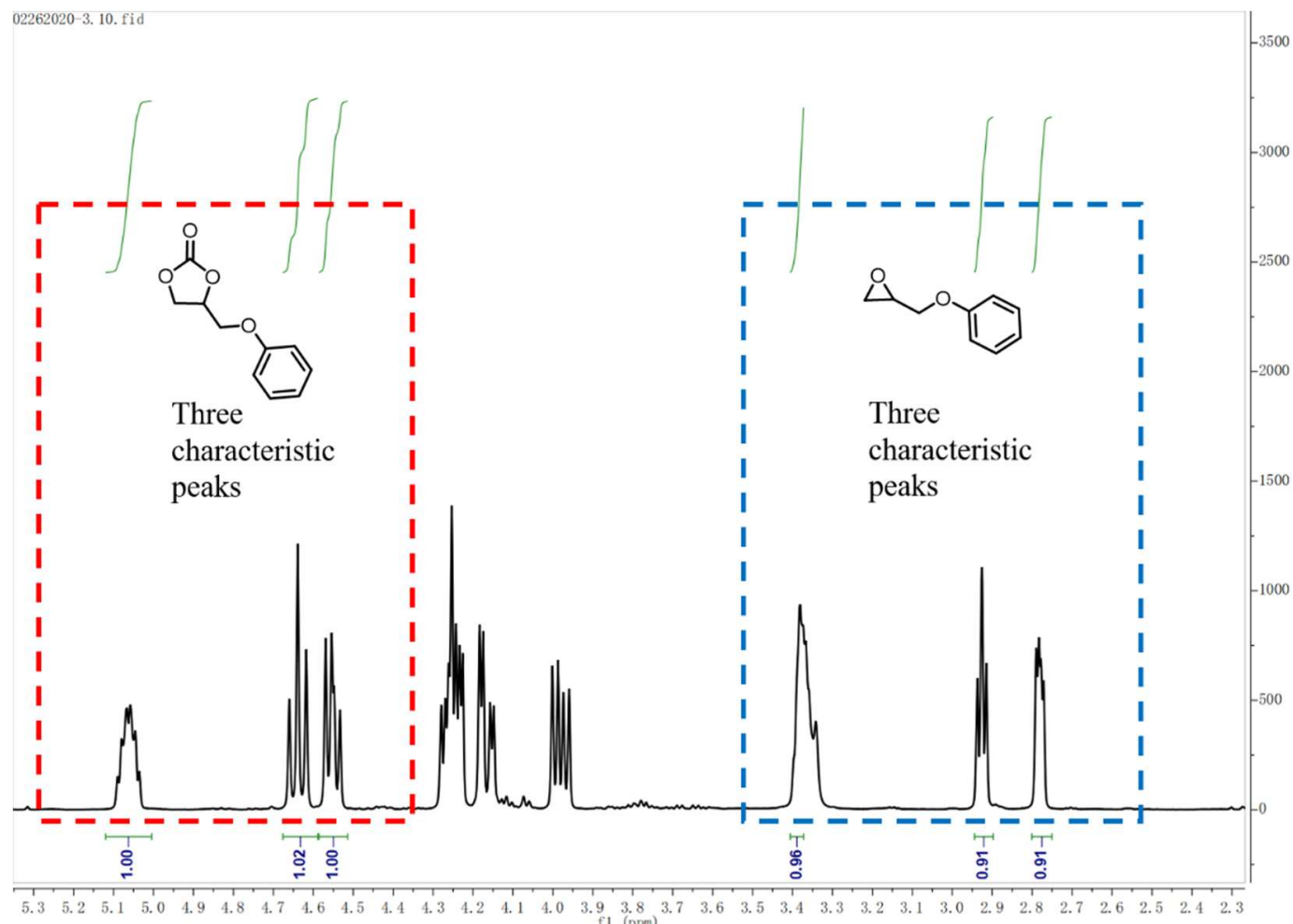

Figure S49. The ${ }^{1}$ H-NMR spectrum of benzyl phenyl glycidyl ether and 3-phenoxy-1,2-propylene carbonate by 5 in $\mathrm{CDCl}_{3}$.

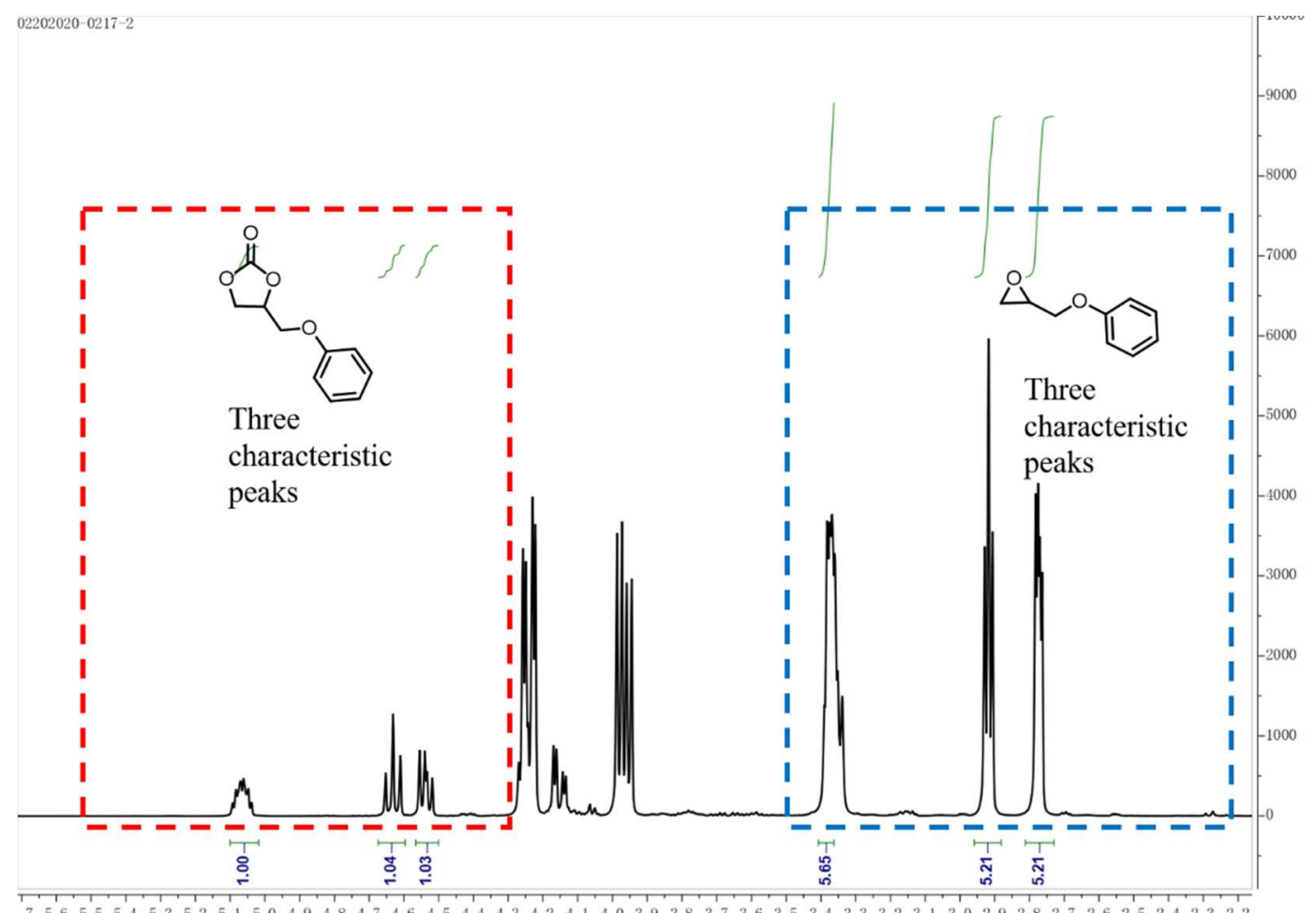

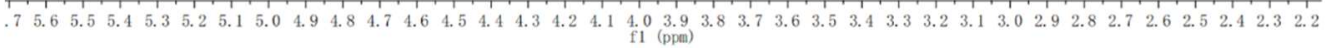

Figure S50. The ${ }^{1} \mathrm{H}-\mathrm{NMR}$ spectrum of benzyl phenyl glycidyl ether and 3-phenoxy-1,2-propylene carbonate by $\mathrm{TBABr}$ without catalyst in $\mathrm{CDCl}_{3}$. 


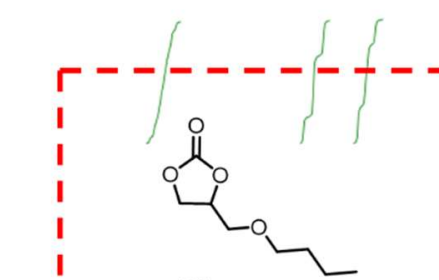

Three characteristic peaks
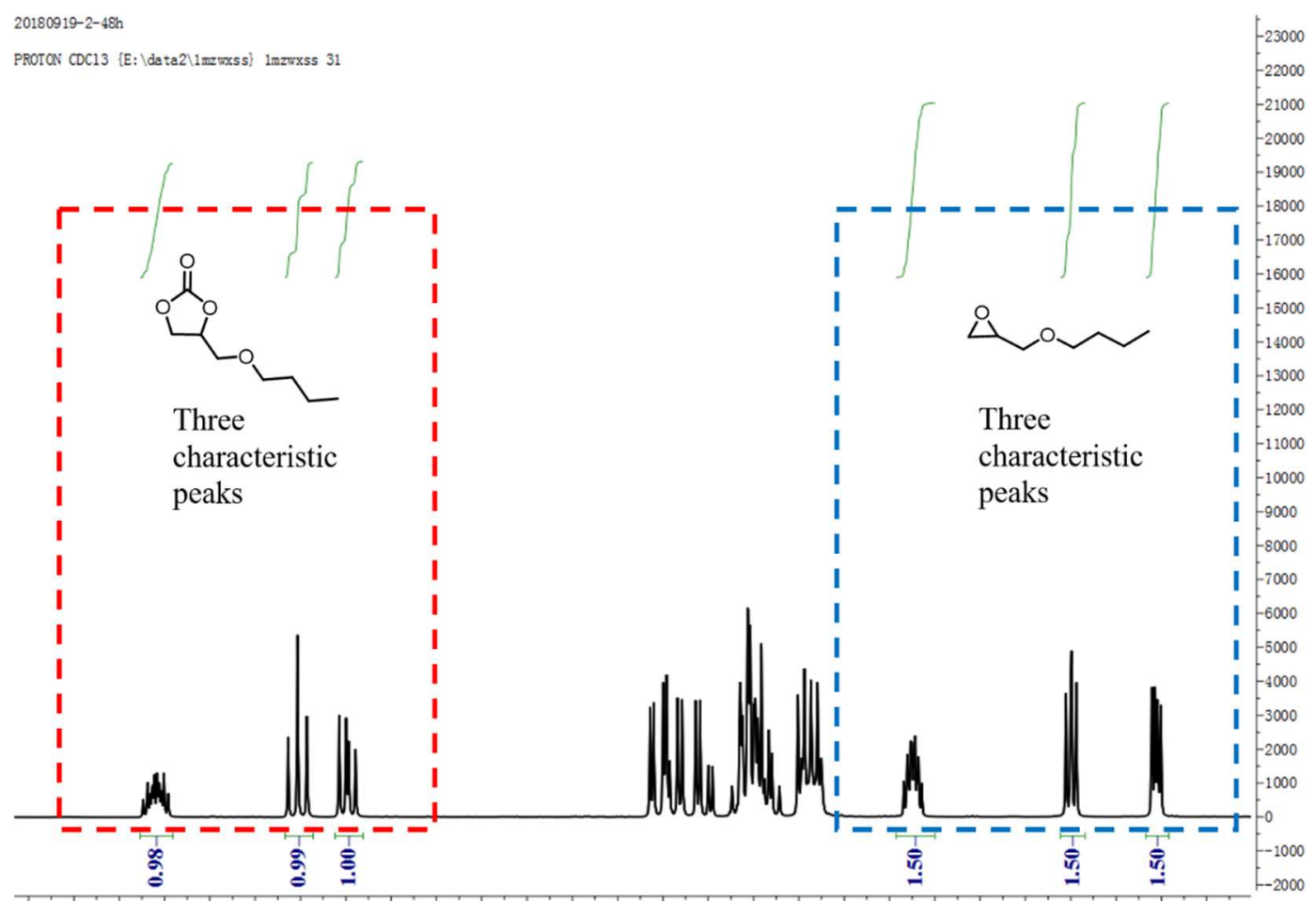

\begin{tabular}{llllllllllllllllllllllllllllllllllllllll}
\hline 5.1 & 5.0 & 4.9 & 4.8 & 4.7 & 4.6 & 4.5 & 4.4 & 4.3 & 4.2 & 4.1 & 4.0 & 3.9 & 3.8 & 3.7 & 3.6 & 3.5 & 3.4 & 3.3 & 3.2 & 3.1 & 3.0 & 2.9 & 2.8 & 2.7 & 2.6 & 2.5
\end{tabular}

Figure S51. The ${ }^{1} \mathrm{H}-\mathrm{NMR}$ spectrum of butyl glycidyl ether and 3-butoxy-1,2-propylene carbonate by 3 in $\mathrm{CDCl}_{3}$.

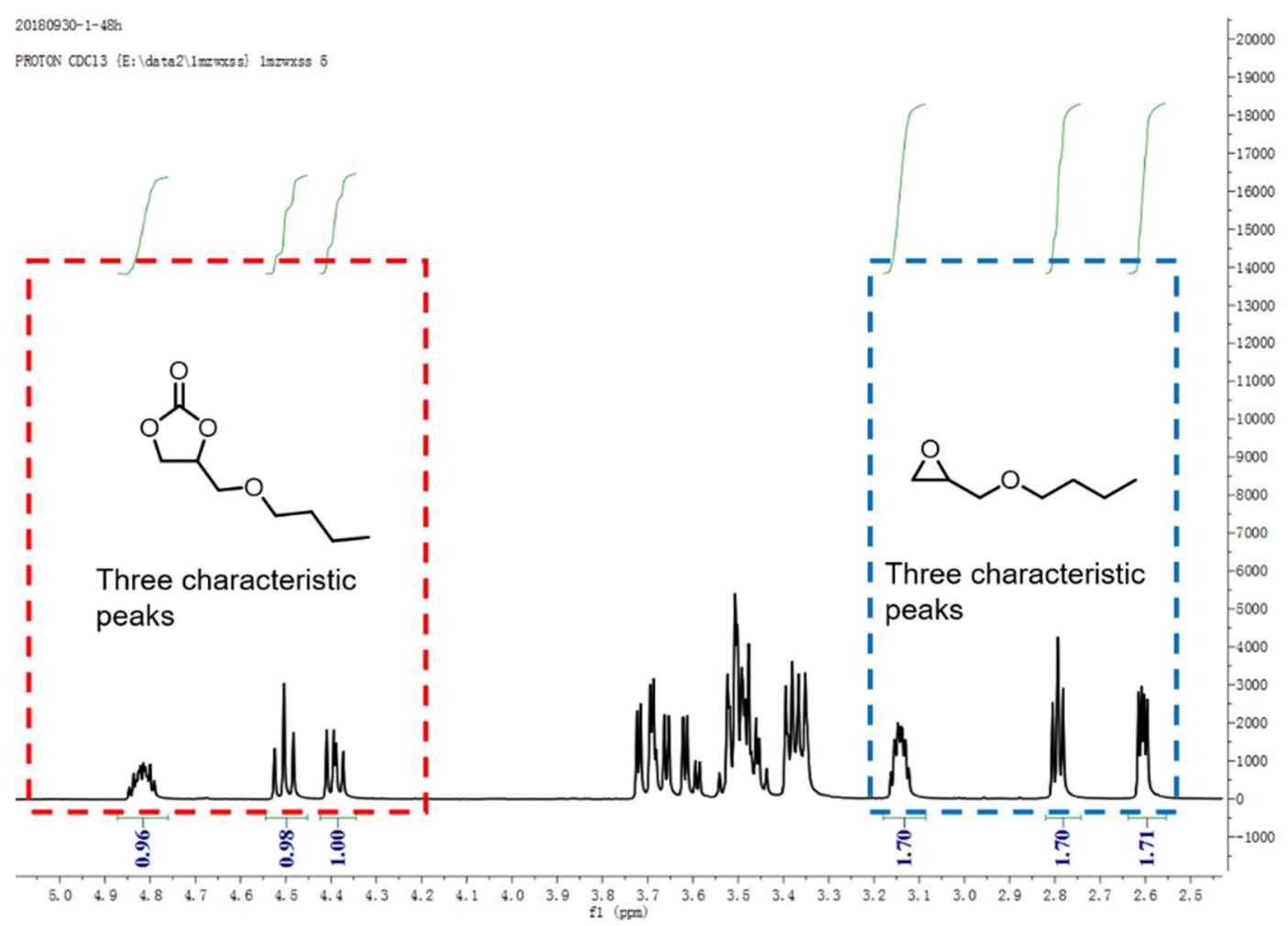

Figure S52. The ${ }^{1} \mathrm{H}-\mathrm{NMR}$ spectrum of butyl glycidyl ether and 3-butoxy-1,2-propylene carbonate by 8$\mathbf{A l}$ in $\mathrm{CDCl}_{3}$. 


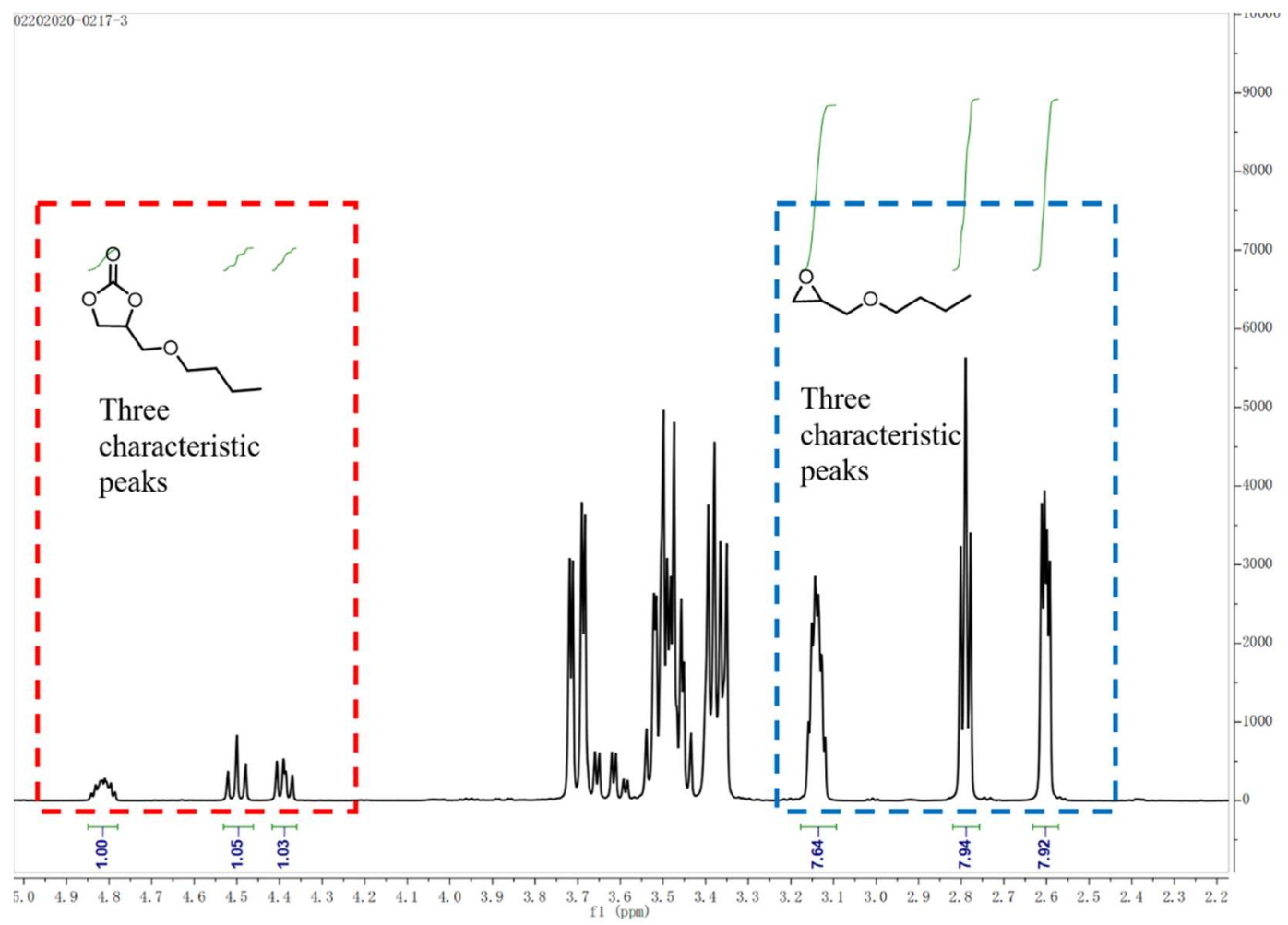

Figure S53. The ${ }^{1} \mathrm{H}-\mathrm{NMR}$ spectrum of butyl glycidyl ether and 3-butoxy-1,2-propylene carbonate by $\mathrm{TBABr}$ without catalyst in $\mathrm{CDCl}_{3}$.

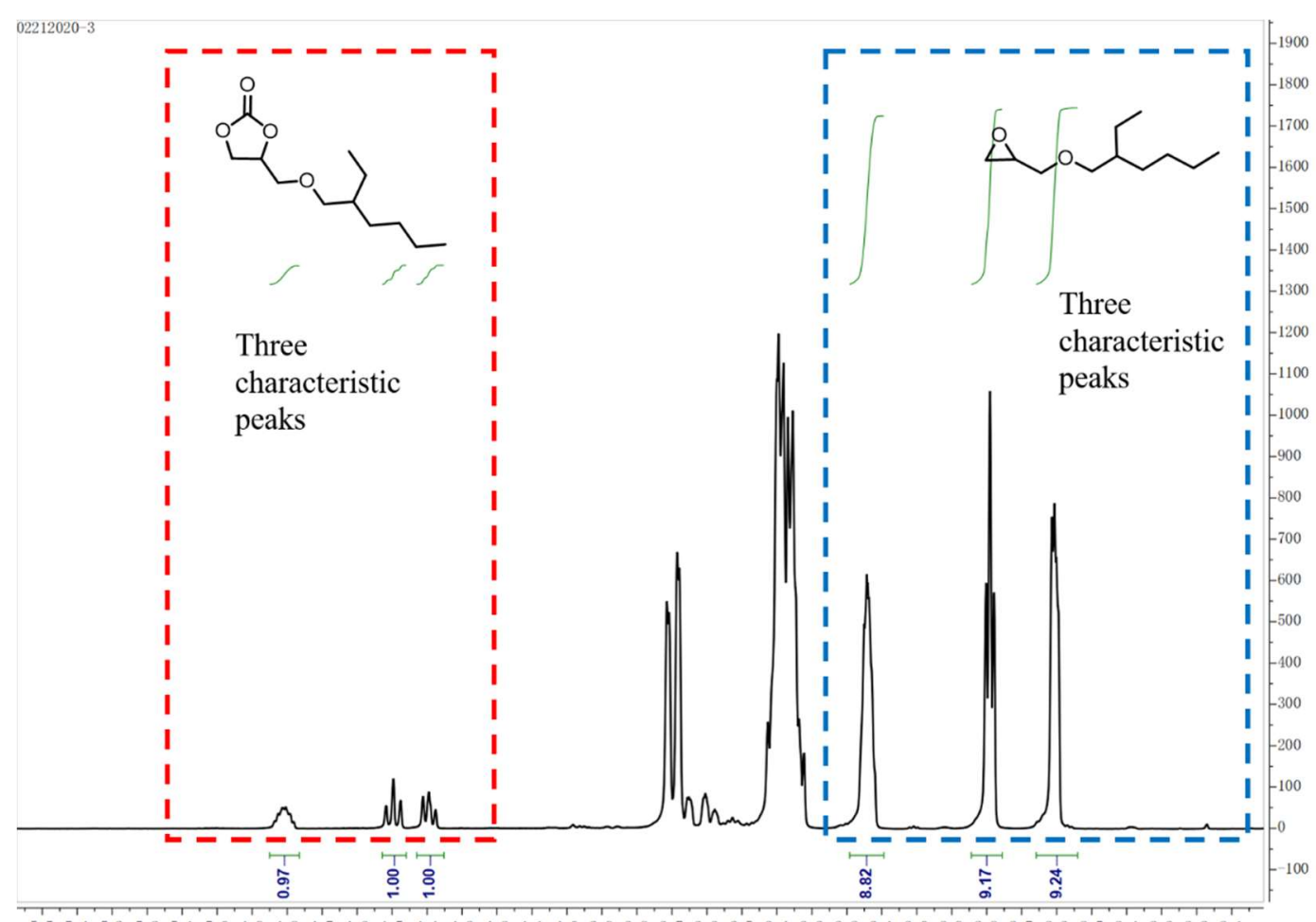

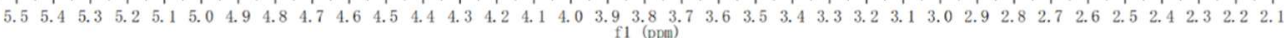

Figure S54. The ${ }^{1} \mathrm{H}-\mathrm{NMR}$ spectrum of 2-Ethylhexyl glycidyl ether and the corresponding carbonate by 3 in $\mathrm{CDCl}_{3}$. 


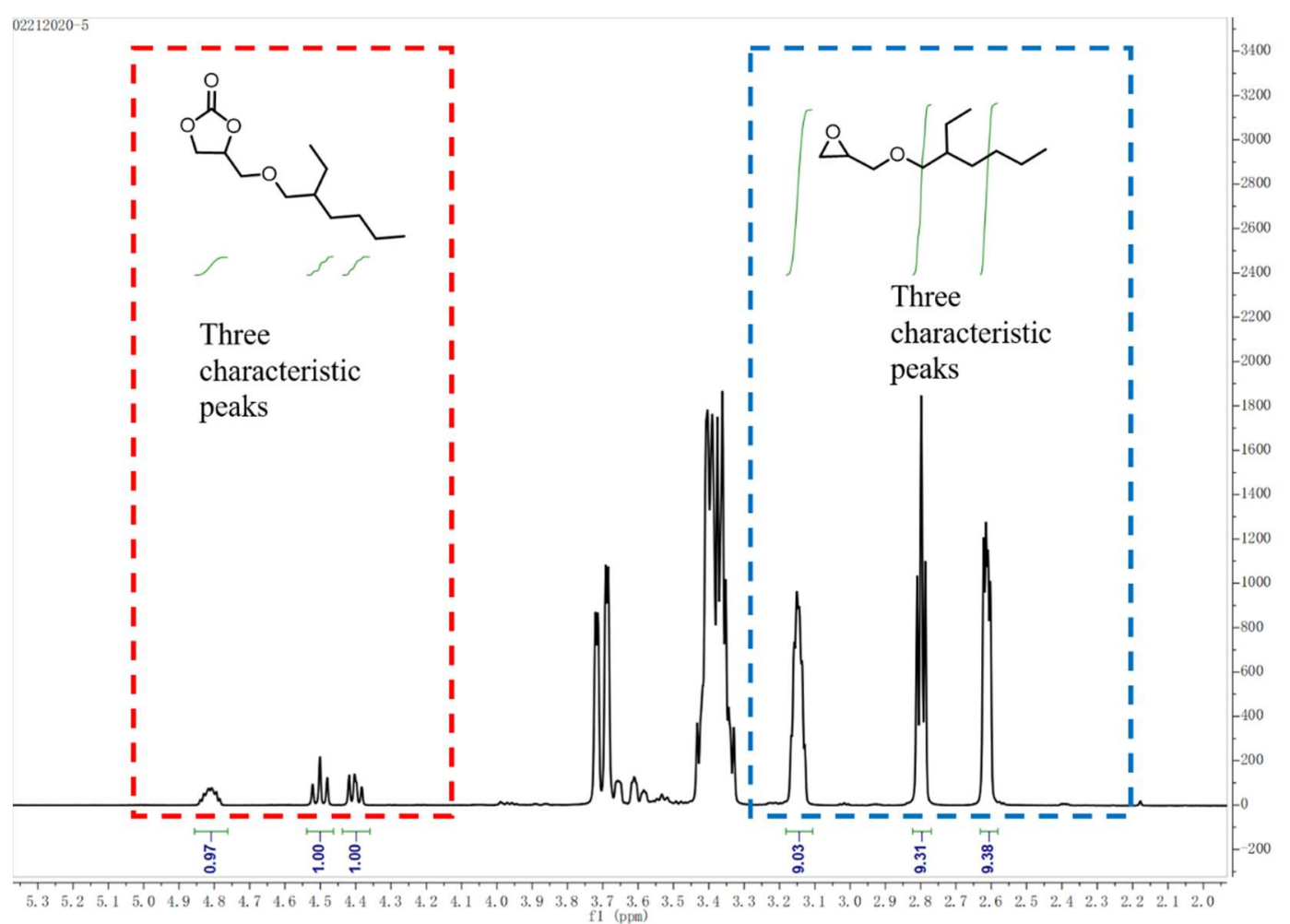

Figure S55. The ${ }^{1} \mathrm{H}-\mathrm{NMR}$ spectrum of 2-Ethylhexyl glycidyl ether and the corresponding carbonate by 8-Al in $\mathrm{CDCl}_{3}$.

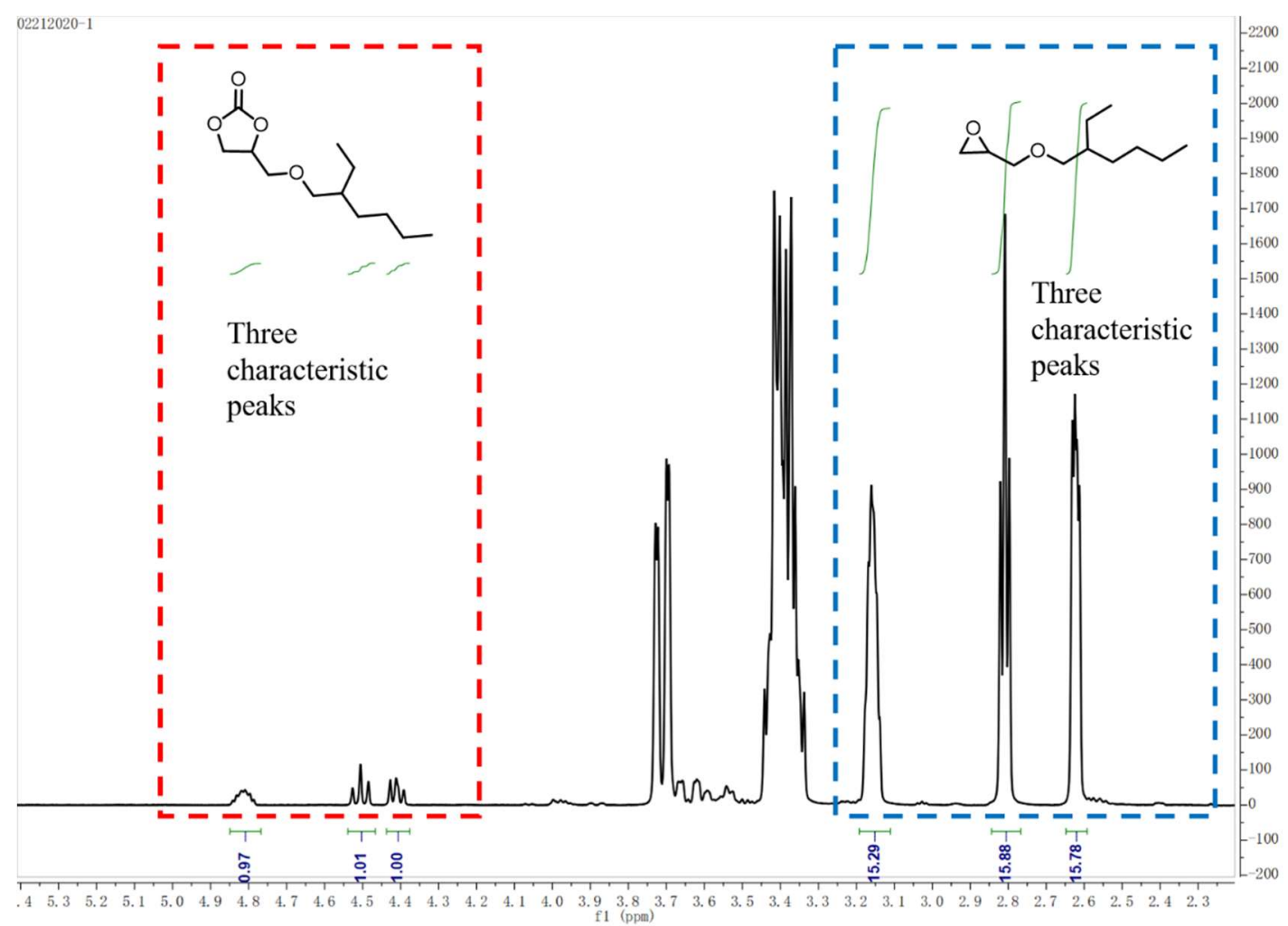

Figure S55. The ${ }^{1} \mathrm{H}-\mathrm{NMR}$ spectrum of 2-Ethylhexyl glycidyl ether and the corresponding carbonate by $\mathrm{TBABr}$ without catalyst in $\mathrm{CDCl}_{3}$. 


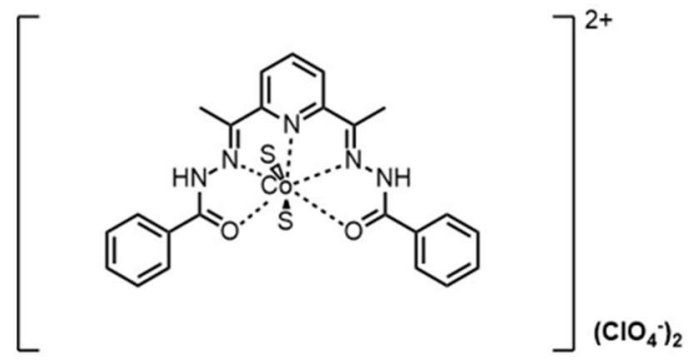

Co(II) mononuclear complex, $\mathrm{S}=\mathrm{CH}_{3} \mathrm{OH}$

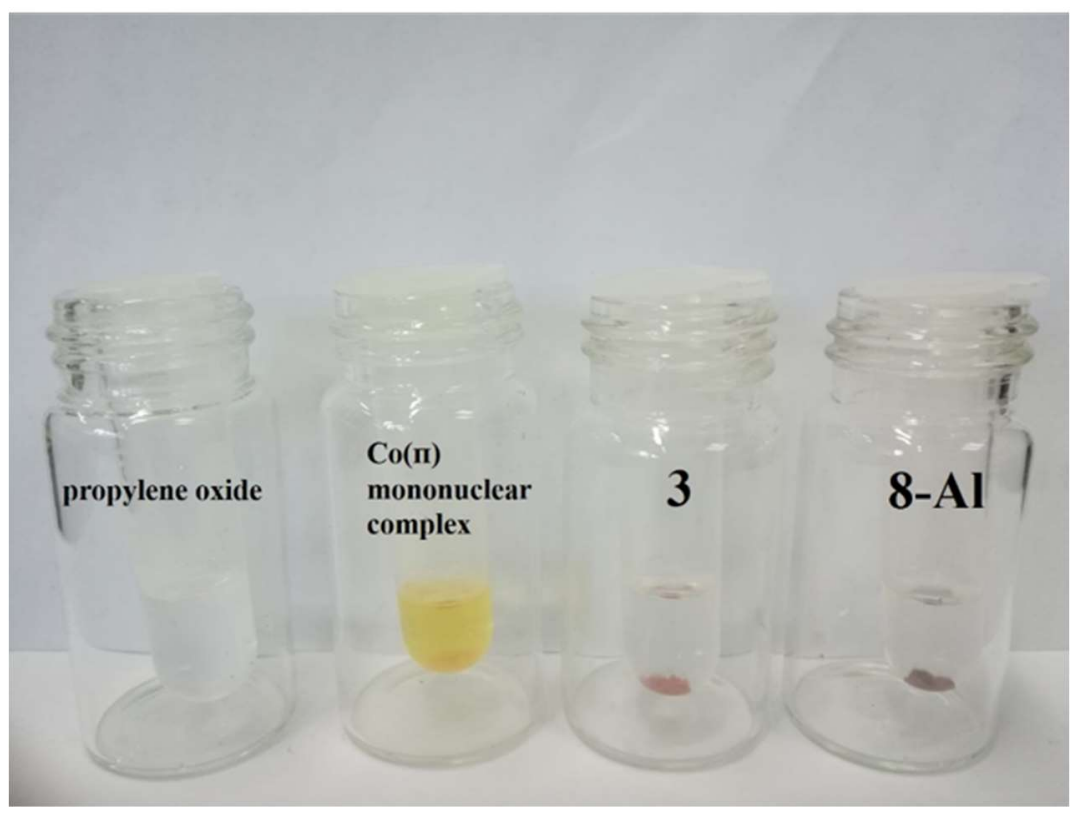

Figure S56. The structure of the related $\mathrm{Co}^{\mathrm{II}}$ mononuclear complex and illustration of its solubility property in comparison to that of $\mathbf{3}$ and $\mathbf{8}-\mathbf{A l}$. 
Table S13. Summary of metallacycles with different Coordination geometry sphere.

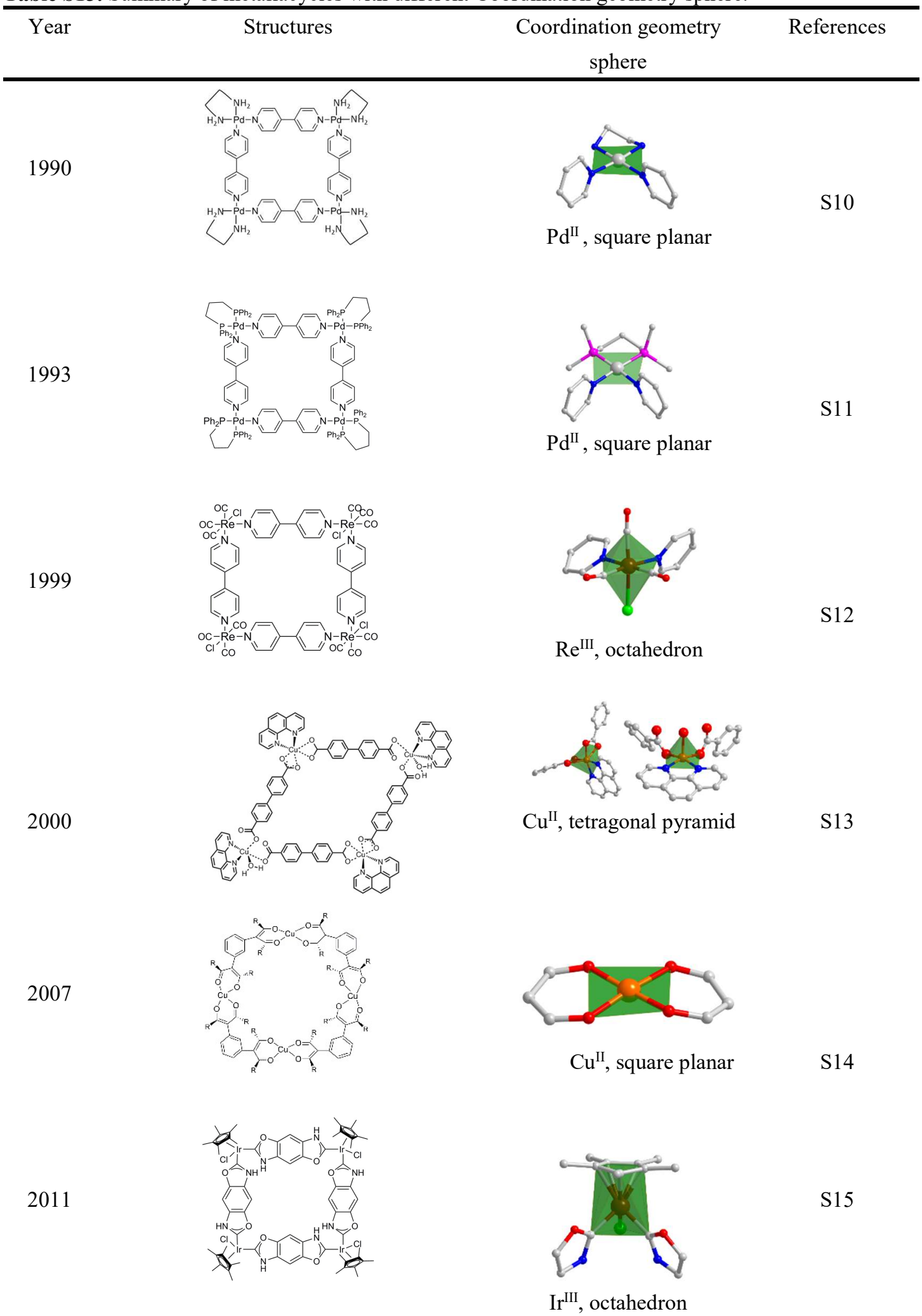


2012
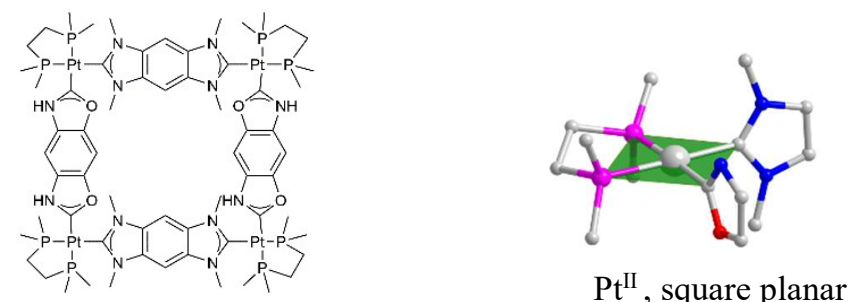

2018
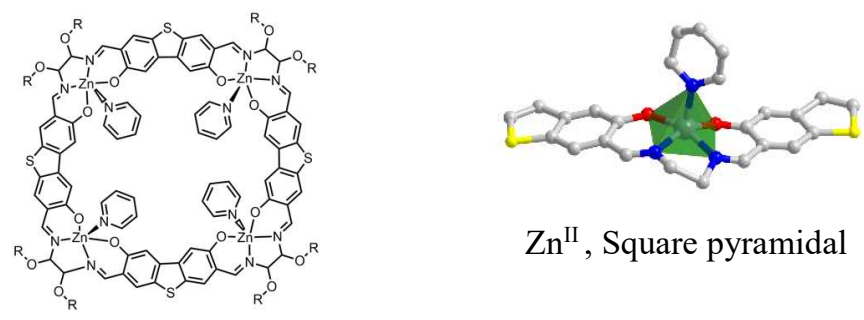

$\mathrm{Zn}^{\mathrm{II}}$, Square pyramidal
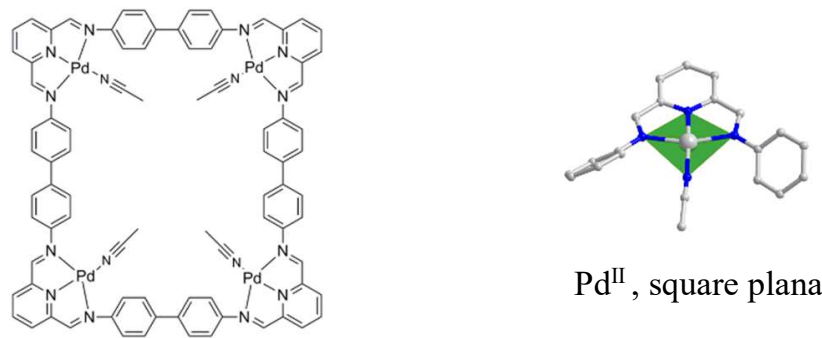

$\mathrm{Pd}^{\mathrm{II}}$, square planar
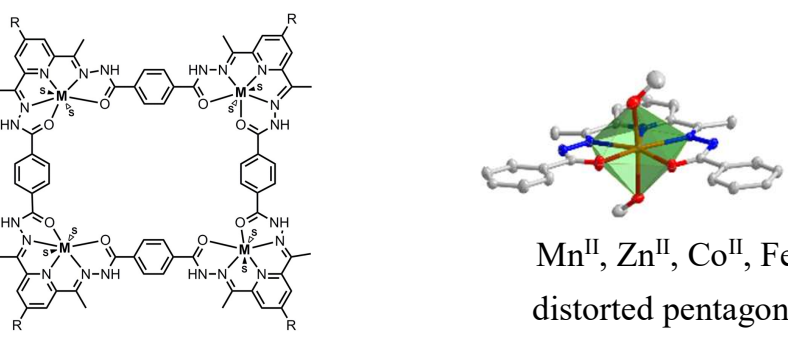

$\mathrm{Mn}^{\mathrm{II}}, \mathrm{Zn}^{\mathrm{II}}, \mathrm{Co}^{\mathrm{II}}, \mathrm{Fe}^{\mathrm{II}}$

this work distorted pentagonal bipyramidal

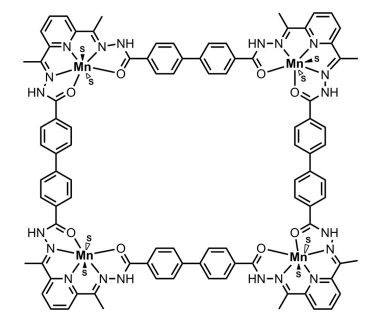

$\mathrm{Mn}^{\mathrm{II}}$

distorted pentagonal

bipyramidal this work 


\section{References}

S1. Zong, R.; Zhou, H.; Thummel, R. P. Direct Access to 4-Carboxy-1,8-naphthyridines and Related Compounds through Pfitzinger-Type Chemistry, J. Org. Chem. 2008, 73, 4334-4337.

S2. Sheldrick, G. M. SADABS: Program for Empirical Absorption Correction of Area Detector Data; University of Göttingen: Göttingen, Germany, 1996.

S3. Sheldrick, G. M. SHELXL 2014. Acta Crystallogr., Sect. C: Struct. Chem. 2015, 71, 3-8.

S4. Johnson, A. M.; Wiley, C. A.; Young, M. C.; Zhang, X.; Lyon, Y.; Julian, R. R.; Hooley, R. J.

Narcissistic Self-Sorting in Self-Assembled Cages of Rare Earth Metals and Rigid Ligands, Angew.

Chem. Int. Ed. 2015, 54, 5641-5645.

S5. Sheldrick, G. M. SADABS 2.05, University Göttingen, Göttingen, Germany, 2002.

S6. Xie, Y.; Wang, T.-T.; Liu, X.-H.; Zou, K.; Deng, W.-Q. Capture and conversion of $\mathrm{CO}_{2}$ at ambient conditions by a conjugated microporous polymer, Nat. Commun. 2013, 4, 1960.

S7. Xie, Y.; Yang, R.-X.; Huang, N.-Y.; Luo, H.-J.; Deng, W.-Q. Efficient fixation of $\mathrm{CO}_{2}$ at mild conditions by a Cr-conjugated microporous polymer, J. Energy Chem. 2014, 23, 22-28.

S8. Ng, C. K.; Toh, R. W.; Lin, T. T.; Luo, H.-K.; Andy Hor, T. S.; Wu, J. Metal-salen molecular cages as efficient and recyclable heterogeneous catalysts for cycloaddition of $\mathrm{CO}_{2}$ with epoxides at ambient conditions, Chem. Sci. 2019, 10, 1549-1554.

S9. Liu, C.; Liu, X.-H.; Li, B.; Zhang, L.; Ma, J.-G.; Cheng, P. Salen-Cu(II)@MIL-101(Cr) as an efficient heterogeneous catalyst for cycloaddition of $\mathrm{CO}_{2}$ to epoxides under mild conditions, J. Energy Chem. 2017, 26, 824-824.

S10. Fujita, M.; Yazaki, J.; Ogura, K. Preparation of a Macrocyclic Polynuclear Complex, [(en)Pd(4,4'bpy) $]_{4}\left(\mathrm{NO}_{3}\right)_{8}$, Which Recognizes an Organic Molecule in Aqueous Media, J. Am. Chem. Soc. 1990, 112, 5645-5647.

S11. Stang, P. J.; Olenyuk, B. Self-Assembly, Symmetry, and Molecular Architecture: Coordination as the Motif in the Rational Design of Supramolecular Metallacyclic Polygons and Polyhedra, Acc. Chem. Res. 1997, 30, 502-518.

S12. Bélanger, S.; Hupp, J. T.; Stern, C. L.; Slone, R. V.; Watson, D. F.; Carrell, T. G. Thin-Film Molecular Materials Based on Tetrametallic "Squares": Nanoscale Porosity and Size-Selective Guest Transport Characteristics J. Am. Chem. Soc. 1999, 121, 557-563.

S13. Liu, G.-F.; Ye, B.-H.; Ling, Y.-H.; Chen, X.-M. Interlocking of molecular rhombi into a 2D polyrotaxane network via $\pi-\pi$ interactions. Crystal structure of $\left[\mathrm{Cu}_{2}(\mathrm{bpa})_{2}(\text { phen })_{2}\left(\mathrm{H}_{2} \mathrm{O}\right)\right]_{2} \cdot 2 \mathrm{H}_{2} \mathrm{O}\left(\mathrm{bpa}^{2-}=\right.$ biphenyl-4,4'-dicarboxylate, phen = 1,10- phenanthroline), Chem. Commun. 2002, 14, 1442-1443.

S14. Pariya, C.; Sparrow, C. R.; Back, C.-K.; Sandí, G.; Fronczek, F. R.; Maverick, A. W. Copper $\beta$ Diketonate Molecular Squares and Their Host-Guest Reactions, Angew. Chem. Int. Ed. 2007, 46, 63056308 .

S15. Conrady, F. M.; Fröhlich, R.; Schulte to Brinke, C.; Pape, T.; Hahn, F. E. Stepwise Formation of a Molecular Square with Bridging NH, O-Substituted Dicarbene Building Blocks, J. Am. Chem. Soc. 2011, 133, 11496-11499.

S16. Schmidtendorf, M.; Pape, T.; Hahn, F. E. Stepwise Preparation of a Molecular Square from NR, 
NR- and NH, O-Substituted Dicarbene Building Blocks, Angew. Chem. Int. Ed. 2012, 51, 2195-2198.

S17. Kawano, S.-i.; Fukushima, T.; Tanaka, K. Specific and Oriented Encapsulation of Fullerene $\mathrm{C}_{70}$ into a Supramolecular Double-Decker Cage Composed of Shape-Persistent Macrocycles, Angew. Chem. Int. Ed. 2018, 57, 14827-14831.

S18. Lavendomme, R.; Ronson, T. K.; Nitschke, J. R. Metal and Organic Templates Together Control the Size of Covalent Macrocycles and Cages, J. Am. Chem. Soc. 2019, 141, 12147-12158. 